\title{
Lehrqualität in der universitären Medizin
}

Hinderliche und förderliche Faktoren guter Lehre aus Sicht der Lehrenden und Darstellung der Implementierung eines innovativen Evaluationskonzepts

\section{Dissertation \\ (kumulatives Verfahren)}

zur Erlangung des humanwissenschaftlichen Doktorgrades

in der Medizin der Georg-August-Universität Göttingen

\section{vorgelegt von \\ Sarah Nina Schiekirka-Schwake}

aus

Marburg 
Dekan:

Prof. Dr. Heyo K. Kroemer

\section{Betreuungsausschuss / Thesis Committee}

Erstbetreuerin (Gutachterin): $\quad$ Prof. Dr. Nicole von Steinbüchel-Rheinwall

Institut für Medizinische Psychologie und

Medizinische Soziologie

Universitätsmedizin Göttingen

Zweitbetreuer (Gutachter): $\quad$ Prof. Dr. Tim Beißbarth

Institut für Medizinische Bioinformatik

Universitätsmedizin Göttingen

Drittbetreuer:

Prof. Dr. Tobias Raupach, MME

Bereich Medizindidaktik und Ausbildungsforschung;

Klinik für Kardiologie und Pneumologie

Universitätsmedizin Göttingen

Weitere Mitglieder der Prüfungskommission

Prof. Dr. Christoph Herrmann-Lingen

Klinik für Psychosomatische Medizin und

Psychotherapie

Universitätsmedizin Göttingen

Prof. Dr. Wolfgang Himmel

Institut für Allgemeinmedizin

Universitätsmedizin Göttingen

Prof. Dr. Jens Wiltfang

Klinik für Psychiatrie und Psychotherapie

Universitätsmedizin Göttingen 
Diese kumulative Schrift gründet sich auf die folgenden Originalarbeiten:

1. Schiekirka-Schwake S, Anders S, von Steinbüchel N, Becker JC, Raupach T (2017): Facilitators of high-quality teaching in medical school: findings from a nation-wide survey among clinical teachers. BMC Med Educ 17, 178

2. Schiekirka-Schwake S, Dreiling K, Pyka K, Anders S, von Steinbüchel N, Raupach T (2018): Improving evaluation at two medical schools. Clin Teach $\underline{15}, 314-318$ 


\section{Erklärung}

Hiermit erkläre ich, die Dissertation mit dem Titel „Lehrqualität in der universitären Medizin - Hinderliche und förderliche Faktoren guter Lehre aus Sicht der Lehrenden und Darstellung der Implementierung eines innovativen Evaluationskonzepts" eigenständig angefertigt und keine anderen als die von mir angegebenen Quellen und Hilfsmittel verwendet zu haben.

Göttingen, 26. Februar 2019 
Im Sinne einer besseren Lesbarkeit wird in dieser Arbeit die männliche oder neutrale Form personenbezogener Hauptwörter gewählt. Dies impliziert keine Benachteiligung eines Geschlechts. 


\section{Inhaltsverzeichnis}

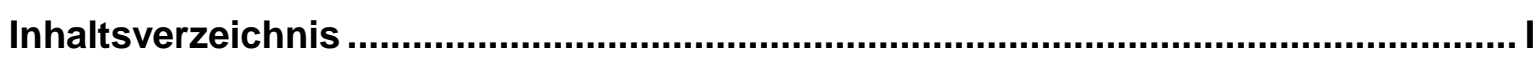

Abbildungsverzeichnis ..................................................................................... II

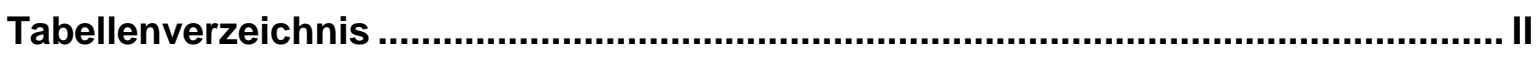

Abkürzungsverzeichnis ........................................................................................ III

1 Einleitung..................................................................................................... 1

2 Stand der Forschung ......................................................................................... 1

2.1 Herausforderungen an die lehrenden Ärzte im klinischen Abschnitt des

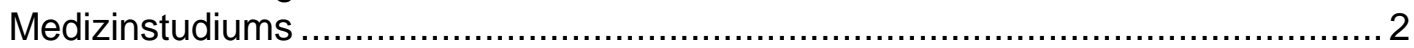

2.2 Evaluation als ein Instrument zur Erreichung einer hohen Lehrqualität ..................5

2.3 Veränderungsmanagement....................................................................... 8

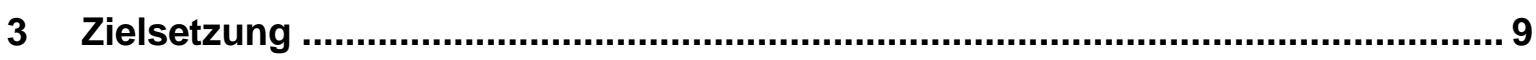

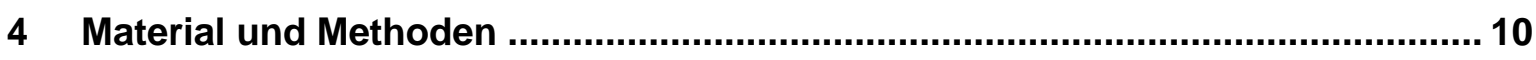

4.1 Erfassung der Lehrbedingungen für klinisch tätige Ärzte.................................. 10

4.2 Untersuchung der Implementierung eines neuen dozentenspezifischen

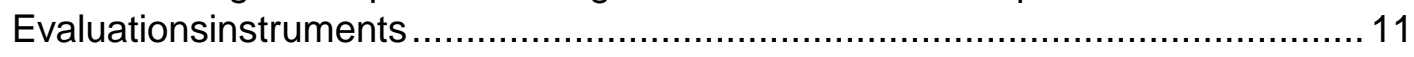

4.3 Datenschutz und Genehmigung der Ethikkommission .................................... 13

5 Kumulative Darstellung der eigenen Arbeiten .................................................. 14

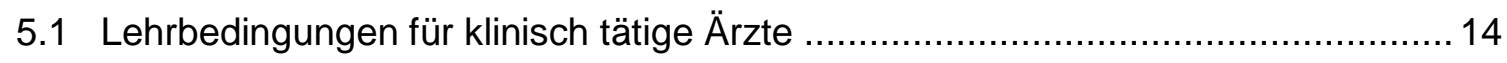

5.2 Implementierung eines neuen dozentenspezifischen Evaluationsinstruments...... 18

5.2.1 Ergebnisse der Fokusgruppen mit den Lehrenden .................................... 19

5.2.2 Ergebnisse der Fokusgruppen mit den Studierenden ................................... 21

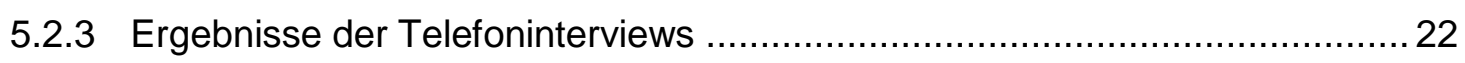

6 Diskussion ................................................................................................. 22

6.1 Diskussion der Ergebnisse der Fragebogenuntersuchung ……………….........2

6.2 Diskussion der Ergebnisse der Implementierungsstudie .................................. 27

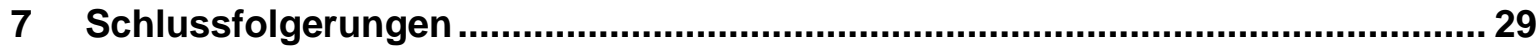

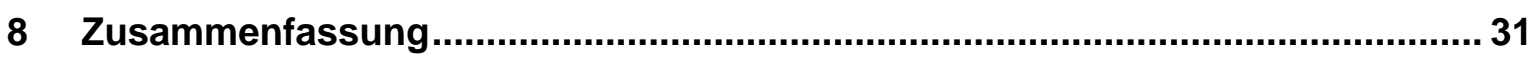

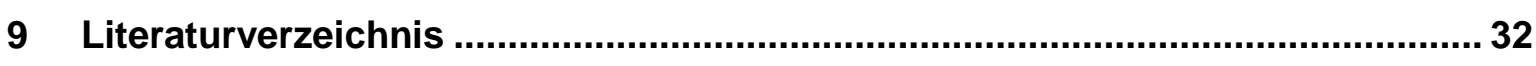

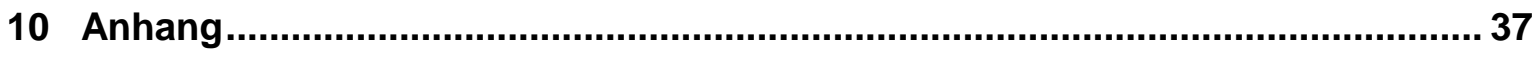

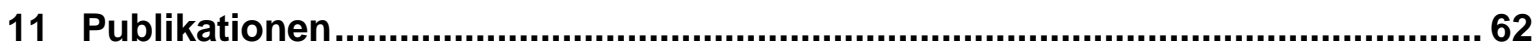




\section{Abbildungsverzeichnis}

Abbildung 1: Darstellung des Projektverlaufs in Göttingen (GÖ) und Hamburg (HH) .... 13

Abbildung 2: Aspekte guter Lehre. Einschätzungen der Wichtigkeit der verschiedenen Aspekte.

Abbildung 3: Hinderliche Faktoren. Einschätzung, inwiefern die Faktoren die Lehrenden bei der Durchführung der eigenen Lehre behindern.

Abbildung 4: Förderliche Faktoren. Einschätzung, inwiefern die Faktoren die Lehrenden bei der Durchführung der eigenen Lehre unterstützen.

\section{Tabellenverzeichnis}

Tabelle 1: Haupt- und Subthemen der Fokusgruppendiskussionen mit den Studierenden und Lehrenden 


\section{Abkürzungsverzeichnis}

$\begin{array}{ll}\text { GÖ } & \text { Göttingen } \\ \mathrm{HH} & \text { Hamburg } \\ \mathrm{M} & \text { Mittelwert } \\ \mathrm{n} & \text { Menge / Anzahl } \\ \mathrm{SD} & \text { Standardabweichung }\end{array}$




\section{Einleitung}

Die studentische Lehre in der universitären Medizin muss hohen Ansprüchen genügen, da diese einen elementaren Baustein in der Ausbildung der zukünftigen Ärzte darstellt. Leider sind die Bedingungen zur Erreichung einer hohen Lehrqualität nicht immer ohne Weiteres gegeben. Ein Großteil der Lehre im klinischen Abschnitt des Medizinstudiums wird von Ärzten durchgeführt, die in der Krankenversorgung tätig sind. Nicht alle diese Ärzte begrüßen, dass sie mit der Anstellung an einem Universitätskrankenhaus auch eine Lehrverpflichtung eingehen (Gerrity et al. 1997). Zudem ist das Absolvieren didaktischer Weiterbildungen an vielen medizinischen Universitäten keine Grundvoraussetzung, um Medizinstudierende zu unterrichten. Doch auch wenn die Ärzte motiviert und didaktisch geschult sein sollten, stellt die Dreifachbelastung aus Patientenversorgung, wissenschaftlichem Engagement sowie der Durchführung studentischer Lehre eine große Herausforderung für die klinisch tätigen Lehrenden dar, und häufig fällt Lehre in der Prioritätenrangreihe gegenüber den anderen Verpflichtungen zurück (Abrahamson 1996; Müller-Hilke 2010; Seabrook 2003).

Das gemeinsame Ziel der in dieser Schrift kumulativ dargestellten Forschungsarbeiten ist es, die Rahmenbedingungen zur Erreichung einer hohen Lehrqualität in der medizinischen Lehre zu eruieren. In der ersten Publikation wird auf die Wahrnehmung klinisch tätiger Lehrender unter Berücksichtigung der akademischen Qualifikation (habilitiert versus nicht habilitiert) fokussiert. Es werden deren Verständnis von guter Lehre sowie hinderliche und förderliche Faktoren für die Erbringung guter Lehrleistung näher beleuchtet. In der zweiten Arbeit wird der Schwerpunkt auf die studentische Lehrevaluation als ein wichtiges Instrument zur Sicherung und Optimierung der Lehrqualität gelegt. Hierbei wird die Implementierung eines neuen dozentenspezifischen Evaluationsfragebogens aus Sicht der Lehrenden sowie der Studierenden untersucht.

\section{Stand der Forschung}

In dem folgenden Kapitel gilt es zunächst, den aktuellen Forschungsstand bezogen auf die Rahmenbedingungen für Lehrende in der Medizin darzustellen. Im Weiteren werden wichtige Erkenntnisse zur studentischen Lehrevaluation, insbesondere bezogen auf die Verwendung in der medizinischen Lehre, näher erläutert. Abschließend soll auf ausgewählte Literatur zum Veränderungsmanagement eingegangen werden, da diese den 
theoretischen Hintergrund für die Implementierung des neuen Evaluationsinstruments liefert.

\subsection{Herausforderungen an die lehrenden Ärzte im klinischen Abschnitt des Medizinstudiums}

Während Dimitroff und Davis 1996 basierend auf einer umfassenden Literaturübersicht noch einen Forschungsbedarf bezogen auf Lehrende in der medizinischen Ausbildung identifizierten, konnte diese Forschungslücke in den vergangenen Jahren weitestgehend geschlossen werden.

Ein Großteil der durchgeführten Studien zeigte, dass Lehrende in der universitären Medizin mit vielen Hindernissen zu kämpfen haben (Arabshahi et al. 2015; Barrett und Scott 2014; Hoffman und Donaldson 2004; Huwendiek et al. 2013; Huwendiek et al. 2010; Sabel und Archer 2014; Seabrook 2003). So äußerten 22 Lehrende in Großbritannien im Rahmen halbstrukturierter Interviews Bedenken bezüglich der unzureichenden institutionellen Unterstützung und Anerkennung von Lehre an Universitätskrankenhäusern sowie die geringen Möglichkeiten, Entscheidungen in der medizinischen Ausbildung zu beeinflussen (Seabrook 2003). Als hinderlichster Faktor bei der Durchführung von Lehre bzw. guter Lehre wurde Zeitmangel benannt. Insbesondere die älteren Studienteilnehmer führten dies auf den in den vergangenen Jahren zunehmenden wirtschaftlichen Druck zurück. Die Ärzte gaben an, dass offiziell zwar Zeit für Lehre eingeplant sei, in der Realität diese jedoch auch für Forschung, administrative Aufgaben sowie unternehmerische Verpflichtungen aufgebracht werden müsse (Seabrook 2003). Zusätzlich dazu bemängelten die Lehrenden die teilweise geringe Lernmotivation der Studierenden. In einer australischen Studie wurden acht klinisch tätige Lehrende interviewt (Barrett und Scott 2014). Hier wurde ebenfalls fehlende Zeit als bedeutender limitierender Faktor benannt und große Besorgnis bezüglich der Qualität der Patientenversorgung geäußert, da die Erfüllung der Lehrverpflichtungen in direkter zeitlicher Konkurrenz zu den klinischen Aufgaben stehe. Des Weiteren berichteten die Ärzte, meist auf die Verwendung innovativer didaktischer Methoden zu verzichten, weil die Zeit für deren Erprobung fehle. In einer weiteren australischen Studie wurden 24 in die studentische Lehre involvierte Pädiater im Rahmen von Fokusgruppendiskussionen befragt (Peadon et al. 2010). Alle Diskussionsteilnehmer gaben an, grundsätzlich Freude am Lehren zu haben, teilten jedoch außerdem mit, dass diese durch Zeitdruck, fehlende eigene Selbstsicherheit in der Lehre sowie Mangel an institutioneller Anerkennung und studentischem Interesse gemindert werde. Knight und Bligh (2006) interviewten 13 Ärzte in Großbritannien. Bei dieser Studie lag der Fokus auf der Wahrnehmung der klinischen Lehre 
insbesondere im Kontext der Veränderungen, die in den letzten Jahren durch Trends wie die Erhöhung klinischer Produktivität oder Hinwendung zu multidisziplinären Ansätzen hervorgerufen wurden. Die Autoren konnten bei einigen Lehrenden einen deutlichen Widerstand gegenüber der veränderten Lehrpraxis identifizieren. Wieder wurde bei der Frage, welche Unterstützung zur Erfüllung der Lehrerrolle benötigt wird, an erster Stelle zusätzliche Zeit genannt. Dybowski und Harendza (2014) untersuchten an einem deutschen Universitätskrankenhaus mittels Fokusgruppen $(n=15)$ verschiedene Faktoren, die die Lehrmotivation klinisch tätiger Ärzte beeinflussen. Neben den individuellen Wertesystemen der Lehrenden wurden studentenbezogene Faktoren, wie beispielsweise die studentische Motivation, sowie strukturelle Faktoren, wie Zeitdruck und fehlende Anerkennung, identifiziert. Finanzielle Anreize spielten für die Lehrenden keine bedeutende Rolle, dafür wurden verschiedene Aspekte bezogen auf die Durchführung der Evaluationen kritisiert. So fehlte den Lehrenden teilweise die individuelle Rückmeldung der Evaluationsergebnisse, andere empfanden es als belastend, ständig der Kritik der Studierenden ausgesetzt zu sein. Arabshahi und Kollegen (2015) fokussierten in einer ebenfalls qualitativ angelegten Studie an einem iranischen Universitätskrankenhaus auf die Lehrveranstaltungsform Unterricht am Krankenbett. Hier sahen sich die neun klinisch tätigen Ärzte auch mit verschiedenen Herausforderungen konfrontiert, wie beispielsweise mit der schlechten Organisation der Lehre auf Station (z. B. keine konstante Betreuung von Studierendengruppen durch einen Lehrenden), der zeitlichen Konkurrenz von Patientenversorgung und Lehre, der fehlenden Motivation der Studierenden sowie der Sorge um das Wohlbefinden der Patienten. Mit klinischer Lehre auf Station beschäftigten sich außerdem Hoffman und Donaldson (2004). Sie analysierten mittels direkter Beobachtung, Fokusgruppen, Einzelinterviews sowie Dokumentenauswertung die Lehre drei klinischer Teams und identifizierten die Patienten als wichtigsten Einflussfaktor. Die Lehr- und Lernaktivitäten auf Station waren beispielsweise abhängig von der Anzahl der Patienten sowie deren Krankheiten. Zusätzlich stellten zeitliche Restriktionen durch Patientenuntersuchungen sowie pflegerische Maßnahmen einen bedeutenden Einflussfaktor dar.

In einer international durchgeführten quantitativen Erhebung gaben die 860 Teilnehmer ein Fehlen von akademischer Wertschätzung, finanzieller Unterstützung sowie didaktischer Weiterbildungen als größte Hindernisse für Lehrende in der Medizin an (Huwendiek et al. 2010). Zeitmangel wurde von den Teilnehmern an vierter Stelle genannt. Zu beachten ist, dass die Untersuchungsstichprobe nicht auf klinisch tätige Ärzte begrenzt war, sondern Dozenten verschiedener Disziplinen und Arbeitssettings (Universitätskrankenhaus versus privater Sektor) umfasste. Im Einklang mit diesen Ergebnissen nannten 147 deutsche medizinische Lehrende geringe akademische Wertschätzung (54\%), unzureichende 
institutionelle (32 \%) bzw. finanzielle Unterstützung (28\%) sowie fehlende Zeit für Lehraufgaben (19\%) als größte Herausforderungen in der medizinischen Ausbildung (Huwendiek et al. 2013).

Einige wenige Studien konnten bisher einen positiven Einfluss von Lehrverpflichtungen auf Ärzte identifizieren. Hartley et al. (1999) führten mit 30 Londoner Allgemeinmedizinern, die in die studentische Lehre involviert waren, halbstrukturierte Interviews durch und berichteten von einem positiven Effekt auf die Einstellung der Lehrenden durch den Kontakt mit den Studierenden. Darüber hinaus wurde der zusätzliche Patientenkontakt im Rahmen der Lehrverpflichtungen und die Abwechslung in der wöchentlichen Routine positiv bewertet. Rutter und Herzberg (2002) fanden in einer größer angelegten Literaturrecherche heraus, dass Ärzte und Zahnärzte häufig Stress empfinden, dieser jedoch durch das Übernehmen einer zusätzlichen Lehrtätigkeit reduziert werden kann. Die Autoren identifizierten weiteren Forschungsbedarf, um die Ergebnisse erklären zu können.

Die zu dem Thema verfügbaren Forschungsarbeiten sind bezüglich der Erhebungsmethoden und Stichproben heterogen. Ein Großteil der Autoren nutzten qualitative Methoden (z. B. Arabshahi et al. 2015; Barrett und Scott 2014; Knight und Bligh 2006; Peadon et al. 2010; Sabel und Archer 2014; Seabrook 2003), mittels derer hilfreiche Informationen ermittelt werden können. In der Regel gründen sich die Ergebnisse aber auf sehr kleine Stichproben, so dass deren Generalisierbarkeit eingeschränkt ist. Die meisten quantitativen Studien zu dem Thema inkludierten verschiedenste medizinische Lehrende wie beispielsweise Grundlagenwissenschaftler, Psychologen, Physiotherapeuten sowie Pflegepersonal (Huwendiek et al. 2013; Huwendiek et al. 2010). Es ist jedoch anzunehmen, dass der berufliche Hintergrund sowie das Arbeitssetting (Universitätskrankenhaus versus privater Sektor) einen Einfluss auf die Wahrnehmung der eigenen Lehrumgebung haben. Demzufolge scheint zur Beantwortung spezifischer Forschungsfragen die Eingrenzung der Stichprobe auf klinisch tätige Lehrende sinnvoll.

Hierbei ist die Lehrtätigkeit für klinisch tätige Ärzte in der universitären Lehre meist nicht auf eine Veranstaltungsform begrenzt. Die Regel sind Veranstaltungen wie Unterricht am Krankenbett, Seminare, fallbasiertes Lernen und Vorlesungen. So sollte bei Studien, die sich mit der Wahrnehmung des Lehrumfeldes beschäftigen, keine Einschränkung auf eine spezifische Veranstaltungsform erfolgen (Arabshahi et al. 2015; Hoffman und Donaldson 2004). Des Weiteren stellt sich die Frage, welchen Einfluss die akademische Qualifikation spielt. Die Ärzte, die an deutschen Universitätskrankenhäusern arbeiten, können den folgenden zwei Gruppen zugeordnet werden: habilitierte und nicht habilitierte Ärzte. Die meisten nicht habilitierten Ärzte befinden sich noch in ihrer Ausbildung zum Facharzt, welche direkt nach der Approbation begonnen wird. Diese Ärzte sind vorrangig in die Krankenversorgung eingebunden, verpflichten sich aber durch die Beschäftigung an einem 
Universitätskrankenhaus auch zu Engagement in der Forschung sowie in der studentischen Lehre. Die Arbeitsbelastung ist meist sehr hoch. Habilitierte Ärzte haben sich durch das Erbringen von Forschungs- sowie Lehrleistung zusätzlich qualifiziert und somit die Lehrberechtigung (Venia Legendi) inne. In der Regel haben sie außerdem bereits die Facharztprüfung absolviert. Die habilitierten Ärzte sind zwar ebenfalls hoher Arbeitsbelastung ausgesetzt, bekleiden jedoch zumeist höherrangige Positionen, die mit mehr Verantwortung, aber vor allem auch mehr Selbstbestimmtheit einhergehen.

Es wird allgemein angenommen, dass klinisch tätige Ärzte im universitären Umfeld nicht lediglich danach streben, exzellente Patientenversorgung und wissenschaftlich hochwertige Forschung durchzuführen, sondern darüber hinaus motiviert sind, Studierenden dazu zu verhelfen, gute Ärzte zu werden. Trotz der oben genannten Forschungsarbeiten sind die Daten zu dem Thema begrenzt. So findet man keine allgemein gültige Definition von guter Lehre, und obwohl einige Anreize und Hindernisse für gute Lehre beschrieben wurden, gibt es keine Daten dazu, ob habilitierte versus nicht habilitierte Ärzte die gleiche Wahrnehmung ihres Lehrumfeldes sowie förderlicher und hinderlicher Faktoren aufweisen.

\subsection{Evaluation als ein Instrument zur Erreichung einer hohen Lehrqualität}

Ein wesentliches Instrument zur Sicherung und Optimierung der Lehrqualität an medizinischen Hochschulen stellt die studentische Lehrevaluation dar (Berk 2013). Hierbei können die Evaluationsergebnisse auf verschiedenen Ebenen für die kontinuierliche Verbesserung der Lehre genutzt werden (Elzubeir und Rizk 2002). So können die Daten für strukturelle, curriculare Entscheidungen herangezogen werden oder Lehrenden als Feedbackquelle dienen. Um aussagekräftige Daten zu erhalten, werden validierte Evaluationsinstrumente benötigt, die es zulassen, spezifische Stärken und konkreten Optimierungsbedarf zu identifizieren. Bei diesen Instrumenten ist jedoch weder immer das dem Instrument zugrunde liegende Konstrukt von guter Lehre eindeutig definiert, noch lassen die bei sehr breit angelegten Definitionen häufig verwendeten Globalnoten eindeutige Schlussfolgerungen zu.

Zusätzlich dazu ist die Interpretation von Globalnoten durch einige konfundierende Faktoren limitiert (Aleamoni 1999; Marsh und Roche 1997; Schiekirka et al. 2015; Schiekirka und Raupach 2015). Verschiedene Variablen können die Evaluationsergebnisse von Lehrveranstaltungen und Dozierenden beeinflussen bzw. verzerren. Als bestätigt gilt, dass ein hohes initiales studentisches Interesse an einem Kursthema zu besseren Evaluationsergebnissen führt (Berger et al. 2003; Dybowski et al. 2017; Raupach et al. 2012). Darüber hinaus zeigten Woloschuk et al. (2011), dass Studierende, die mit der 
Prüfung im Anschluss einer Veranstaltung zufriedener waren, den Kurs positiver bewerteten. Auch die Ergebnisse von Dozentenevaluationen können verzerrt werden. Demnach werden Lehrende in der Regel besser beurteilt, wenn ihnen ein guter Ruf vorauseilt (Griffin 2001), oder sie besonders unterhaltsam auftreten (Naftulin et al. 1973). Um die Ergebnisse von Evaluationsinstrumenten vor dem Hintergrund potenzieller Einflussbzw. Biasfaktoren interpretieren zu können, ist es wesentlich, das dem Instrument zugrunde liegende Lehrkonstrukt zu kennen (Schiekirka und Raupach 2015). Je nach definiertem Konstrukt kann ein und dieselbe Variable als valider Einflussfaktor wirken oder aber die Ergebnisse verzerren. Wird beispielsweise gute Lehre so definiert, dass Unterhaltsamkeit einen Aspekt derselbigen darstellt, würde die benannte Eigenschaft des Lehrenden nicht als Bias wirken, sondern einen validen Einflussfaktor darstellen.

Problematisch ist außerdem, dass vorhandene Evaluationsinstrumente in der Regel nicht alle Dimensionen der Lehre, nämlich die curricularen Strukturen und Ressourcen, den Lehrprozess, Charakteristika der Lehrenden sowie das Lehrergebnis berücksichtigen (Gibson et al. 2008). Insbesondere für die Bewertung der Ergebnisdimension stehen bisher nur wenige valide Instrumente zur Verfügung. So entwickelten Raupach und Kollegen (2011) ein Evaluationsinstrument, welches die Messung des in einer Lehrveranstaltung erreichten Lernerfolgs in Prozent zulässt. Hierbei schätzen die Studierenden ihren Leistungsstand lernzielorientiert vor und nach Teilnahme an der Veranstaltung auf einer Skala von 1 (trifft voll zu) bis 6 (trifft überhaupt nicht zu) ein. Durch die wiederholte Einschätzung findet das Vorwissen der Studierenden bei der Abschätzung des Lernerfolgs Berücksichtigung. Die genaue Berechnungsmethode des prozentualen Lernerfolgs ist an anderer Stelle ausführlich beschrieben (Raupach et al. 2011). Das Instrument wurde darüber hinaus umfassend validiert (Raupach et al. 2012; Schiekirka et al. 2014; Schiekirka et al. 2013).

Ferner sollten aufgrund der Besonderheiten der medizinischen universitären Lehre medizinspezifische Evaluationsinstrumente herangezogen werden. Nur in der Medizin findet man beispielsweise die Veranstaltungsform Unterricht am Krankenbett, auch werden Lehrveranstaltungen nicht selten von mehreren Lehrenden abgehalten, und die Studierenden haben meist wenig Wahlmöglichkeiten bezogen auf verschiedene Lehrveranstaltungen (Kogan und Shea 2007). Eine Forschergruppe beschäftigte sich kürzlich mit der Entwicklung eines medizinspezifischen Evaluationsinstruments, welches die individuelle prozedurale und strukturelle Lehrleistung von Lehrenden basierend auf den Stanfordkriterien klinischer Lehre erfassen soll (Dreiling et al. 2017; Müller et al. 2017). Dem „Stanford Faculty Development Program“ liegen lerntheoretische Aspekte sowie empirische Beobachtungen von Lehrenden zugrunde. Es benennt folgende Kategorien: Etablierung des Lernklimas, Leitung einer Lehreinheit, Zielkommunikation, Förderung von Verstehen 
und Behalten, Evaluation, Feedback und Förderung selbstbestimmten Lernens (Iblher et al. 2011; Skeff 1988). Die Autoren entwickelten, pilotierten und validierten für die drei Veranstaltungsformen Unterricht am Krankenbett (Dreiling et al. 2017) sowie Klein- und Großgruppenunterricht (Müller et al. 2017) jeweils einen separaten Fragebogen. Die Fragebögen haben eine Batterie an Items gemein, unterscheiden sich jedoch in einigen veranstaltungsrelevanten Fragen.

Die Kombination des soeben vorgestellten Instruments und des weiter oben eingeführten Lernerfolgsinstruments (Raupach et al. 2011; Schiekirka et al. 2014; Schiekirka et al. 2013) scheint eine umfassende, multidimensionale Bewertung individueller Lehrleistung zuzulassen und lässt vermuten, dass die Lehrenden die Ergebnisse zur Sicherung und Optimierung der eigenen Lehrqualität heranziehen können. Dennoch bleibt bisher unklar, inwiefern dies in der Praxis umgesetzt werden kann (Wibbecke et al. 2015). Vor allem für die Lehrenden im klinischen Abschnitt der universitären Medizin scheint es durch die Dreifachbelastung aus Klinik, Forschung und Lehre eine große Herausforderung, die Lehrleistung kontinuierlich auf Basis von individuellen Evaluationsergebnissen zu verbessern.

Generell finden sich lediglich wenige Studien in der Evaluationsforschung, die sich mit der Wahrnehmung der Lehrenden von Evaluation beschäftigen. Schmelkin et al. (1997) zeigten mittels Fragebogenuntersuchung, an der 440 Lehrende verschiedener Disziplinen einer amerikanischen Universität teilnahmen, auf, dass die Einstellung gegenüber studentischer Lehrevaluation grundsätzlich positiv ist, identifizierten jedoch weiteren Forschungsbedarf. Aburawi und Kollegen (2014) adaptierten den Fragebogen von Schmelkin et al. (1997) und nutzte diesen zur Befragung von Lehrenden sowie Studierenden an einer medizinischen Fakultät in den Vereinigten Arabischen Emiraten. Hierbei zeigte sich, dass sowohl die Lehrenden $(n=52)$ als auch die Studierenden $(n=80)$ Evaluation grundsätzlich als ein wichtiges Qualitätssicherungsinstrument wahrnahmen, jedoch beispielsweise bezogen auf die Transparenz des Evaluationsprozesses noch Optimierungsbedarf herrschte. Dybowski und Harendza (2014) fanden, wie bereits oben beschrieben, in der an einem deutschen Universitätskrankenhaus durchgeführten Studie diskrepante Daten zu dem Thema: einige Lehrende wünschten sich individuelles Feedback zu ihren Evaluationsergebnissen, andere berichteten von einem negativen Einfluss der studentischen Kritik auf ihr persönliches Wohlbefinden.

Daten von Studierenden wurden hingegen in sehr vielen Evaluationsstudien erhoben. Diese waren zumeist quantitativer Natur (z. B. Albanese et al. 1997; Berger und Schleußner 2003; Dreiling et al. 2017; Marsh 1983; Schiekirka et al. 2014). Nur wenige Studien verwendeten qualitative Methoden. Billings-Gagliardi und Kollegen (2004) ließen 24 Medizinstudierende einer amerikanischen Universität einen typischen 
Evaluationsfragebogen ausfüllen. Während des Ausfüllens sollten die Studierenden „laut denken“, um herauszufinden, wie die Fragen verstanden und worauf Entscheidungen begründet werden. Die Ergebnisse zeigten, dass die Entscheidungsfindung der Studierenden auf teilweise unerwartete Kriterien zurückzuführen war, Begriffe in Items verschieden verstanden und manche Skalenoptionen willkürlich ausgewählt wurden. Schiekirka et al. (2012) untersuchten mittels Fokusgruppen $(n=17)$ die Wahrnehmung von deutschen Medizinstudierenden bezogen auf die Lehrqualität und die Praxis der Lehrevaluation. Die Studierenden hatten ein breites Verständnis von guter Lehre und begriffen Evaluation als wichtiges Instrument der Qualitätssicherung. Entscheidend für die Lehrqualität wurden jedoch die einzelnen Lehrenden wahrgenommen. Entsprechend wünschten sich die Studierenden, dass die Konsequenzen, die aus den Evaluationsergebnissen gezogen werden, insbesondere an die Lehrenden adressiert würden.

Zum Abgleich der Wahrnehmungen von Studierenden und Lehrenden scheint es demnach sinnvoll bei der Implementierung eines neuen dozentenspezifischen Evaluationsinstruments, Daten von den Evaluierenden als auch den Evaluierten zu erheben.

\subsection{Veränderungsmanagement}

Veränderungen sind allgegenwärtig und spielen für jedes Unternehmen bzw. jede Organisation eine bedeutende Rolle (Al-Haddad und Kotnour 2015; By 2005). Trotz dessen wird in der Literatur für die Implementierung von Veränderungen nicht selten eine Erfolgsrate von unter 30 \% genannt (Beer und Nohria 2000; Burnes 2004). Aufgrund der großen Variabilität der Organisationen, in die Veränderungen implementiert werden, sowie der Veränderungsinitiativen selbst, herrscht Uneinigkeit über das optimale Vorgehen (Bamford und Forrester 2003; Burnes und Jackson 2011). Implementierungsprozesse sollten immer im Kontext der jeweiligen Rahmenbedingungen gesehen werden (Bland et al. 2000; Gale und Grant 1997; Michel et al. 2013). Dabei scheinen multidimensionale Ansätze sinnvoll: Berücksichtigt werden sollten neben dem Inhalt der Veränderungen die involvierten Personen sowie der Prozess (Anderson und Ackermann Anderson 2001). Während des Veränderungsprozesses spielt eine ausgeprägte Kommunikation sowie regelmäßiger Informationsaustausch mit den involvierten Personen eine wichtige Rolle, um die Bemühungen zum Erfolg zu führen (Dannefer et al. 1998; van den Heuvel et al. 2014). Handlungsempfehlungen spezifisch für Implementierungsbemühungen im medizinischen

Kontext haben Gale und Grant (1997) zusammengestellt. Auch sie betonen die Bedeutsamkeit der Kommunikation mit den beteiligten Personen, um ein Klima der 
Kooperation zu schaffen. So möchten insbesondere Mediziner in geplante Veränderungen mit eingebunden werden, ohne das Gefühl zu haben, dass innen Veränderungen diktiert werden. Außerdem sollte aufgrund der Wissenschaftlichkeit von Medizin ein großer Wert auf die Kommunikation rationaler Outcome-Variablen gelegt werden. Ferner betonen die Autoren die Wichtigkeit einer kritischen Erfolgsevaluation der eingeführten Veränderungen im Nachgang.

Bland et al. (2000) fokussierten in ihrem Literaturreview ebenfalls auf den medizinischen Kontext. Die Autoren untersuchten die Frage, wie curriculare Veränderungen erfolgreich an medizinischen Fakultäten implementiert werden können. Als einer der bedeutendsten Einflussfaktoren wurde die für den Veränderungsprozess zuständige Leitung bzw. Leitungsperson und deren Führungsverhalten identifiziert. Des Weiteren benannten die Autoren u. a. die Organisationsstruktur, eine effektive Kommunikation während des Prozesses, ein kooperatives Klima, vorhandene Personalentwicklungsstrategien sowie eine abschließende Evaluation als relevant.

Die Forschungsergebnisse deuten darauf hin, dass die Implementierung eines neuen Evaluationsinstruments mit einigen Schwierigkeiten einhergehen könnte.

In der vorliegenden Arbeit sollte während des Implementierungsprozesses ein großer Wert auf einen hohen Informationsfluss gelegt werden. Des Weiteren sollte durch die Erhebung qualitativer Daten von Lehrenden sowie Studierenden eine umfassende Evaluierung des Implementierungsprozesses ermöglicht werden.

\section{Zielsetzung}

Ziel der ersten hier kumulativ dargestellten Arbeit war es, zum einen das Verständnis klinisch tätiger Lehrender von guter Lehre mittels quantitativer Methoden zu eruieren sowie hinderliche und förderliche Faktoren für die Erbringung guter Lehrleistung zu identifizieren. Zum anderen sollte die Wahrnehmung von Lehrevaluation sowie die Motivation zur Teilnahme an didaktischen Weiterbildungen untersucht werden. Hierbei sollte der Einfluss der akademischen Qualifikation (nicht habilitiert versus habilitiert) auf die Bewertung der klinisch tätigen Lehrenden Berücksichtigung finden.

Die zweite hier kumulativ dargestellte Arbeit hatte das Ziel, zu untersuchen, ob ein dozentenspezifisches Evaluationsinstrument, welches die individuelle prozedurale und strukturelle Lehrleistung sowie den individuell erreichten Lernerfolg erfasst, den Zweck der Sicherung und Optimierung der Lehrqualität erfüllen kann. Darüber hinaus sollte der Implementierungsprozess dieses umfassenden Evaluationsinstruments aus Sicht der Lehrenden sowie Studierenden näher beleuchtet werden. 


\section{Material und Methoden}

Im Folgenden wird auf das methodische Vorgehen der zwei Forschungsarbeiten näher eingegangen. Zuerst wird die quantitative Datenerhebung der Lehrbedingungen für klinisch tätige Ärzte sowie deren statistische Analyse erläutert. Im Weiteren wird der Implementierungsprozess des neuen dozentenspezifischen Evaluationsinstruments, die Erhebung und Auswertung der Fokusgruppen sowie der Telefoninterviews, die im Anschluss an den Prozess durchgeführt wurden, beschrieben.

\subsection{Erfassung der Lehrbedingungen für klinisch tätige Ärzte}

Da für die oben genannte Zielsetzung bisher kein geeignetes Erhebungsinstrument existierte, entwickelte die Autorin der vorliegenden Arbeit in Zusammenarbeit mit Kollegen einen neuen Fragebogen. Als erster Schritt wurden im Frühjahr 2014 an der medizinischen Fakultät Göttingen qualitative Daten im Rahmen von vier Fokusgruppendiskussionen mit fünf bis sieben Teilnehmern erhoben. Um eventuelle divergierende Sichtweisen von nicht habilitierten und habilitierten Ärzten zu erfassen, wurden zwei Gruppen ausschließlich mit nicht habilitierten ( $n=15$; davon männlich $=9$, weiblich $=6$ ) und zwei Gruppen mit habilitierten Ärzten ( $n=11$; alle männlich) durchgeführt. Die Auswertung der qualitativen Daten wurde mittels MAXQDA (VERBI GmbH, Marburg, Deutschland), orientiert an der qualitativen Inhaltsanalyse nach Mayring (Mayring 2010), durchgeführt.

Auf Grundlage der Ergebnisse der Fokusgruppenanalysen erfolgte als zweiter Schritt die Erstellung der ersten Fragebogenversion, die anschließend basierend auf Diskussionen mit Fragebogenexperten und der Durchführung von kognitiven Debriefings mit fünf klinisch tätigen Ärzten (weiblich $=3$, männlich $=2$; nicht habilitiert $=3$, habilitiert $=2$ ) noch geringfügig verändert wurde.

Im Januar 2015 wurde der Onlinefragebogen (EvaSys, Electric Paper, Lüneburg, Deutschland) an der medizinischen Fakultät Göttingen pilotiert. Insgesamt konnten 183 Datensätze von klinisch tätigen Ärzten zur Analyse herangezogen werden, somit belief sich der Rücklauf auf $26 \%$. Von den 183 Personen waren 114 nicht habilitiert (männlich $=57$, weiblich $=57$ ) und 69 habilitiert (männlich $=49$, weiblich $=14$, fehlend $=6$ ). Aufgrund von Deckeneffekten, die bei einigen Items auftraten, wurde der Fragebogen erneut leicht angepasst und Priorisierungsfragen hinzugefügt. Der finale Fragebogen enthielt 34 skalierte Items (5-er Likert-Skala;1 kennzeichnete die negativste und 5 die positivste Antwort) sowie 26 kategoriale Items und zwei offene Fragen. Es wurden Fragen zur Person, zur Idealvorstellung von guter Lehre, zu Faktoren, die die Lehrenden bei der Durchführung ihrer Lehre einerseits behindern sowie andererseits unterstützen, zu Erfahrungen mit 
didaktischen Weiterbildungen und der Nützlichkeit von Evaluationsergebnissen für die Verbesserung der eigenen Lehrleistung gestellt. Der Fragebogen ist in Anhang A angefügt. In dem Zeitraum von September 2015 bis Juli 2016 fand die Datenerhebung an neun medizinischen Fakultäten (Aachen, Dresden, Greifswald, Hamburg, Mainz, Marburg, München, Münster, Oldenburg) statt. Der Link zu dem Onlinefragebogen (EvaSys, Electric Paper, Lüneburg, Deutschland) wurde zusammen mit dem Einladungstext und Informationen über das Projekt durch die jeweiligen Studiendekanate per E-Mail an die klinisch tätigen Ärzte ihrer Fakultäten gesendet. Alle teilnehmenden Studiendekanate nutzten zum Versenden des Einladungsschreibens umfassende Verteilerlisten, die die Kontaktdaten verschiedenster Fakultätsmitglieder enthielten. Bedauerlicherweise konnte die genaue Anzahl der klinisch tätigen Ärzte, die in der Lehre tätig (also die Grundgesamtheit der Zielgruppe) sind, nicht aus den Verteilern erschlossen werden, so dass kein Rücklauf ermittelt werden konnte.

Die Auswertung erfolgte zum einen deskriptiv: Es wurden Prozentzahlen sowie Mittelwerte (M) und Standardabweichungen (SD) berechnet. Zum anderen wurden für die statistischen Vergleiche der Gruppen (nicht habilitierte versus habilitierte Ärzte) inferenzstatistische Verfahren (unabhängiger t-Test, $\chi^{2}$-Test) angewandt. Das Signifikanzlevel wurde auf $5 \%$ festgesetzt. Waren die Mittelwertsunterschiede im t-Test signifikant, wurde zur Bestimmung der Größe des Effekts Cohen's d berechnet. Für die Interpretation von Cohens's d gelten folgende Richtwerte: kleiner Effekt $=0,20$ bis 0,50 ; mittlerer Effekt $=0,51$ bis 0,80 ; großer Effekt $=$ über 0,80 (Rost 2005). Aufgrund der explorativen Natur der Datenanalyse wurde keine Bonferroni-Korrektur vorgenommen.

Cohen's d wurde mit G*Power 3.1 (Düsseldorf, Deutschland) berechnet. Alle weiteren Berechnungen erfolgten mit dem Softwarepaket SPSS ${ }^{\circledR}$ (IBM Corp., Armonk, USA).

\subsection{Untersuchung der Implementierung eines neuen dozentenspezifischen Evaluationsinstruments}

Das Forschungsvorhaben wurde an den zwei deutschen medizinischen Fakultäten Göttingen und Hamburg durchgeführt. Zuerst wurden Dozenten, vorrangig aus dem klinischen Abschnitt des Medizinstudiums, dazu eingeladen, sich mit dem neuen dozentenspezifischen Evaluationsinstrument evaluieren zu lassen. In Anhang B bis D sind drei exemplarische Evaluationsfragebögen, jeweils einer für eine der drei Veranstaltungsformen (Seminar, Unterricht am Krankenbett, Vorlesung) angefügt. Vor der Implementierung des neuen Evaluationsinstruments fand die Evaluation an den Fakultäten in der Regel global am Ende der Veranstaltungsreihe statt, ohne dass die Lehrenden oder Veranstaltungen separat erfasst und bewertet wurden. 
Die Information der beteiligten Lehrenden und Studierenden über das Projekt und dessen Ablauf erfolgte an verschiedenen Stellen: Die Verantwortlichen für die Veranstaltungsreihen wurden sowohl per E-Mail als auch im persönlichen Gespräch über das Projekt informiert. Sie wurden darum gebeten, die Lehrenden der jeweiligen Veranstaltungsreihen ebenfalls über die Studie in Kenntnis zu setzen. Anschließend wurden den Verantwortlichen sowie den Lehrenden weitere Informationen per E-Mail zugesendet. Personen, die nicht auf die E-Mails antworteten, wurden darüber hinaus per Telefon kontaktiert.

Die Studierenden erhielten die Informationen ebenfalls per E-Mail. Zusätzlich wurden sie von Mitgliedern des Forschungsprojekts während der Lehrveranstaltungen über das Projektvorhaben mündlich aufgeklärt und zur Teilnahme gebeten. Im Rahmen dessen erhielten sowohl die Lehrenden als auch die Studierenden die Informationen nochmals in Papierform. Die Datenerhebung fand am Ende der Veranstaltungen statt und wurde von den Projektmitgliedern durchgeführt.

Die Evaluationsrückmeldungen bzw. Feedbackreports für die einzelnen Lehrenden unterschieden sich an den zwei Fakultäten. Die Göttinger Lehrenden erhielten, wie ursprünglich angestrebt, Rückmeldungen zu ihrer individuell durchgeführten Lehre sowie Daten zu der durchschnittlichen Lehrleistung aller Lehrenden der gleichen Lehrveranstaltung. Die Lehrenden in Hamburg erhielten aufgrund der Entscheidung der Verantwortlichen vor Ort nur die aggregierten Daten und konnten somit keine Rückschlüsse auf ihre individuelle Lehrleistung ziehen.

Im Anschluss an die Implementierungsphase des neuen Evaluationsinstruments wurden die Lehrenden, die mit dem Instrument evaluiert worden waren, sowie die teilnehmenden Studierenden eingeladen, an Fokusgruppendiskussionen teilzunehmen. Es wurden insgesamt vier Fokusgruppen mit Lehrenden durchgeführt. Drei der Gruppen fanden in Hamburg statt ( $n=12$; davon männlich = 8, weiblich = 4) und eine in Göttingen $(n=4$; alle männlich). Da in Göttingen vier Lehrende aus terminlichen Gründen nicht an den Fokusgruppen teilnehmen konnten, wurden diese vier Personen (alle männlich) in Einzelinterviews befragt. Mit den Studierenden konnten fünf Fokusgruppen durchgeführt werden. Drei in Göttingen ( $n=21$; davon männlich = 11, weiblich =10) und zwei in Hamburg ( $n=12$; davon männlich = 7, weiblich $=5$ ). Die Erhebungen fanden im April 2015 statt. Die Leitfragen thematisierten das Ziel von Evaluation im Allgemeinen, die bisher durchgeführte Evaluation, die Evaluation mit dem neuen Instrument sowie die Motivation der Studierenden und Lehrenden. Bei den Lehrenden wurde außerdem noch auf die Ergebnisrückmeldung eingegangen.

Basierend auf den Ergebnissen der Fokusgruppen- und Einzelinterviews mit den Lehrenden wurden die individuellen Feedbackreports für die zweite Erhebungsrunde etwas verändert. In den Reports wurden nun auch die Freitextkommentare aufgeführt. Außerdem 
wurden Farbkodierungen hinzugefügt, um den Lehrenden auf einen Blick deutlich zu machen, ob ihre individuellen Ergebnisse besonders gut ausgefallen sind (grün) oder Verbesserungspotential besteht (rot). Ein beispielhaftes Exemplar für einen Feedbackreport ist in Anhang $\mathrm{E}$ zu finden. Des Weiteren erhielten die Lehrenden ein individuelles Anschreiben, in dem ihre spezifischen Stärken und Schwächen bzw. Verbesserungspotenziale aufgeführt wurden (siehe Anhang $\mathrm{F}$ ).

Im Anschluss an die zweite Datenerhebungsrunde (April bis Juli 2015) und nachdem die Lehrenden die angepassten Feedbackreports erhalten hatten, nahmen 43 Dozenten ( männlich $=34$, weiblich $=9$ ) von 56 kontaktierten Personen an kurzen Telefonbefragungen teil. In diesen gaben sie Rückmeldungen zu den Feebackreports im Allgemeinen und den neuen Anpassungen im Speziellen. Die Telefonbefragungen wurden in dem Zeitraum von Oktober bis Dezember 2015 durchgeführt.

Der Projektverlauf ist in Abbildung 1 zusammengefasst.

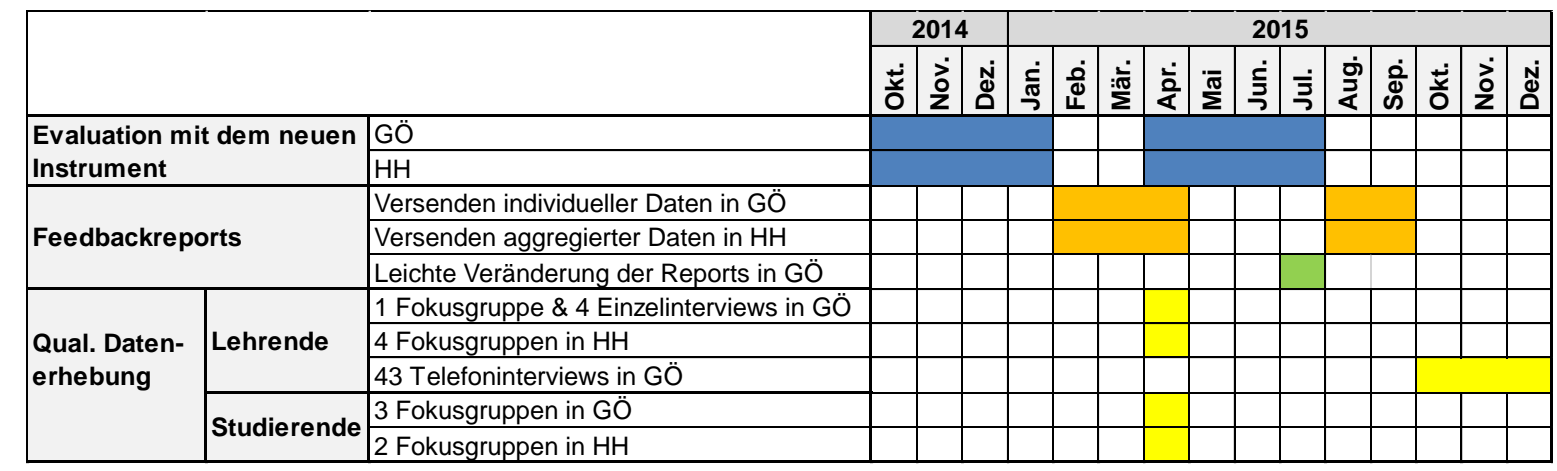

Abbildung 1: Darstellung des Projektverlaufs in Göttingen (GÖ) und Hamburg (HH).

Die Fokusgruppendiskussionen sowie die Einzelinterviews wurden aufgezeichnet und im Anschluss wörtlich transkribiert. Die Daten wurden mittels MAXQDA (VERBI GmbH, Marburg, Deutschland) basierend auf der qualitativen Inhaltsanalyse nach Mayring (Mayring 2010) ausgewertet. Die Ergebnisse der Telefoninterviews wurden im Anschluss an die Telefonate schriftlich zusammengefasst und anhand der Leitfragen ausgewertet.

\subsection{Datenschutz und Genehmigung der Ethikkommission}

Die Ethikkommission der Universitätsmedizin Göttingen hatte sowohl für das erste hier dargestellte Forschungsprojekt (Antragsnummer 20/4/14) als auch für das zweite (Antragsnummer 21/4/14) keine ethischen oder rechtlichen Bedenken. Die Ethikkommission des Universitätsklinikums Hamburg entschied ebenso (Antragsnummer WF-033/1). 
Alle teilnehmenden Personen wurden vor Erhebung der Daten schriftlich über das jeweilige Forschungsvorhaben informiert, auch wurden die Einwilligungen zur Teilnahme schriftlich eingeholt. Die Teilnahme an den Erhebungen erfolgte freiwillig, das Einverständnis konnte jederzeit ohne Nachteile für die Person widerrufen werden. Alle Daten wurden unter strenger Beachtung des Datenschutzes erhoben und vor der Auswertung anonymisiert.

\section{Kumulative Darstellung der eigenen Arbeiten}

Im Folgenden werden die wichtigsten Ergebnisse der zwei hier kumulativ dargestellten Arbeiten zusammenfassend beschrieben. Zuerst werden die Resultate der bundesweiten Fragebogenerhebung dargestellt. Gruppenunterschiede werden lediglich bei signifikantem t- bzw. $\chi^{2}$-Test berichtet.

Im Weiteren wird auf die Erkenntnisse eingegangen, die im Rahmen der qualitativen Datenerhebung mit den Lehrenden sowie den Studierenden gewonnen werden konnten.

\subsection{Lehrbedingungen für klinisch tätige Ärzte}

Schiekirka-Schwake S, Anders S, von Steinbüchel N, Becker JC, Raupach T (2017): Facilitators of high-quality teaching in medical school: findings from a nation-wide survey among clinical teachers. BMC Med Educ $\underline{17}, 178$

Insgesamt konnten 833 der 1035 Datensätze zur Analyse herangezogen werden. Von diesen waren 569 Personen nicht habilitiert (männlich $=313$, weiblich $=245$, fehlend $=11$ ) und 264 (männlich 187, weiblich $=71$, fehlend $=6$ ) waren habilitiert.

Die meisten Items, mittels derer der Ideal-Zustand guter Lehre eingeschätzt werden sollte, wurden von den Befragten als wichtig bis sehr wichtig eingeschätzt. In Abbildung 2 sind die Ergebnisse im Detail dargestellt. 


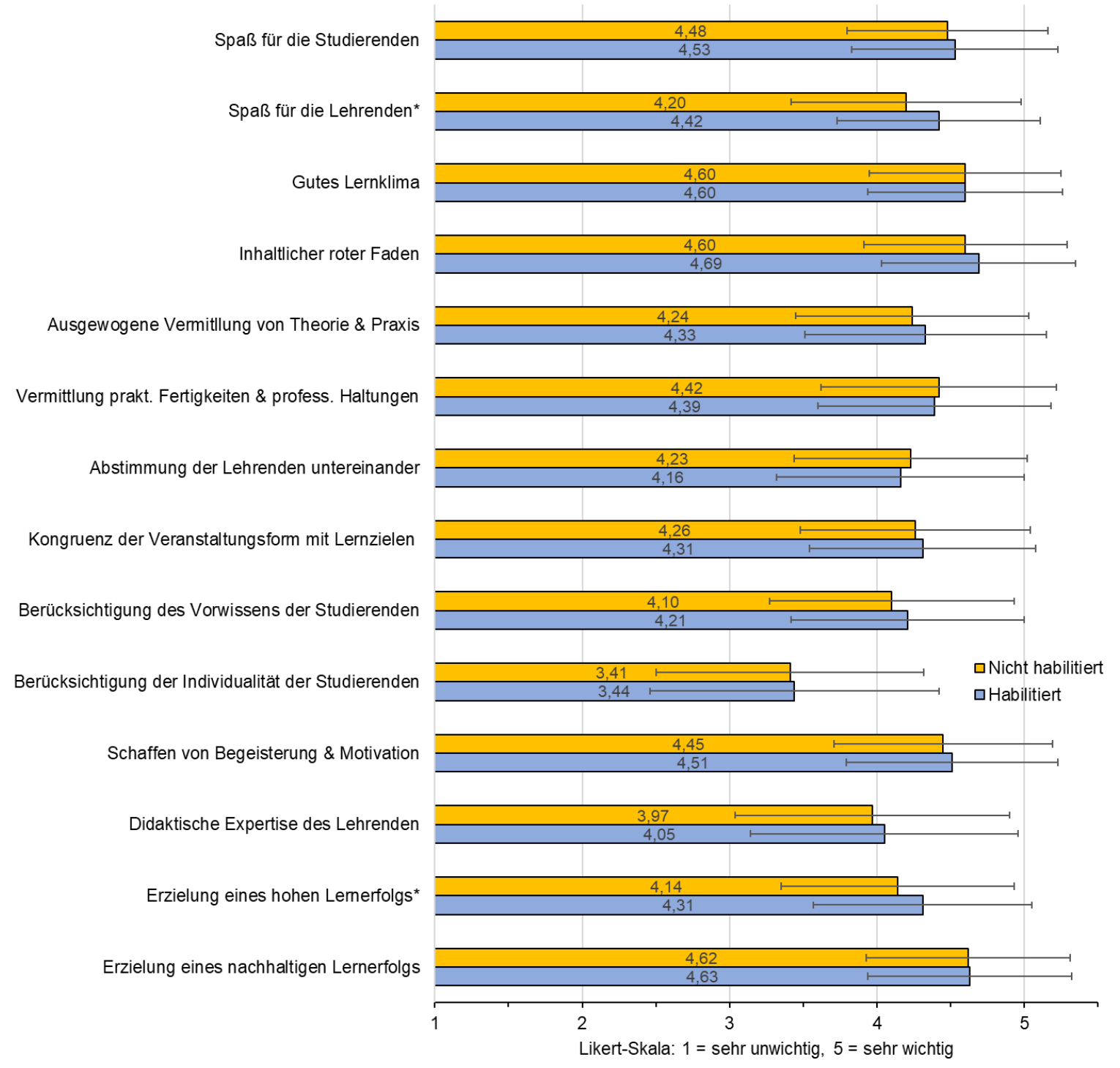

Abbildung 2: Aspekte guter Lehre. Einschätzungen der Wichtigkeit der verschiedenen Aspekte. Balken repräsentieren die Mittelwerte, Fehlerbalken die Standardabweichungen (getrennt für die nicht habilitierten und habilitierten Lehrenden). ${ }^{*} p<0,05$ für den Gruppenvergleich zwischen den nicht habilitieren und habilitierten Lehrenden (unabhängiger t-Test).

Signifikante Unterschiede zwischen den Gruppen mit kleinen Effektstärken fanden sich lediglich bei den Aspekten „Spaß für die Lehrenden“ $(t(830)=3,94 ; p<0,001$; Cohen's $d=$ $-0,29)$ und "Erzielung eines hohen Lernerfolgs" $(t(829)=2,94 ; p<0,001$; Cohen's $d=$ $-0,23)$. In beiden Fällen bewerteten die habilitierten Lehrenden die Items als wichtiger.

Bei der Priorisierung der Aspekte nannten $38 \%$ der Befragten an erster Stelle „Erzielung eines nachhaltigen Lernerfolgs", gefolgt von „Der/die Lehrende schafft bei den Studierenden Begeisterung und Motivation für das Thema." (16 \%) und „Der Unterricht macht den Studierenden Spaß." (12\%). 
Bezogen auf die tatsächliche Lehrsituation der Befragten wurde „Fehlende Vorbereitungszeit“ als größtes Hindernis bei der Durchführung der eigenen Lehre eingestuft. Die genauen Daten sind Abbildung 3 zu entnehmen.

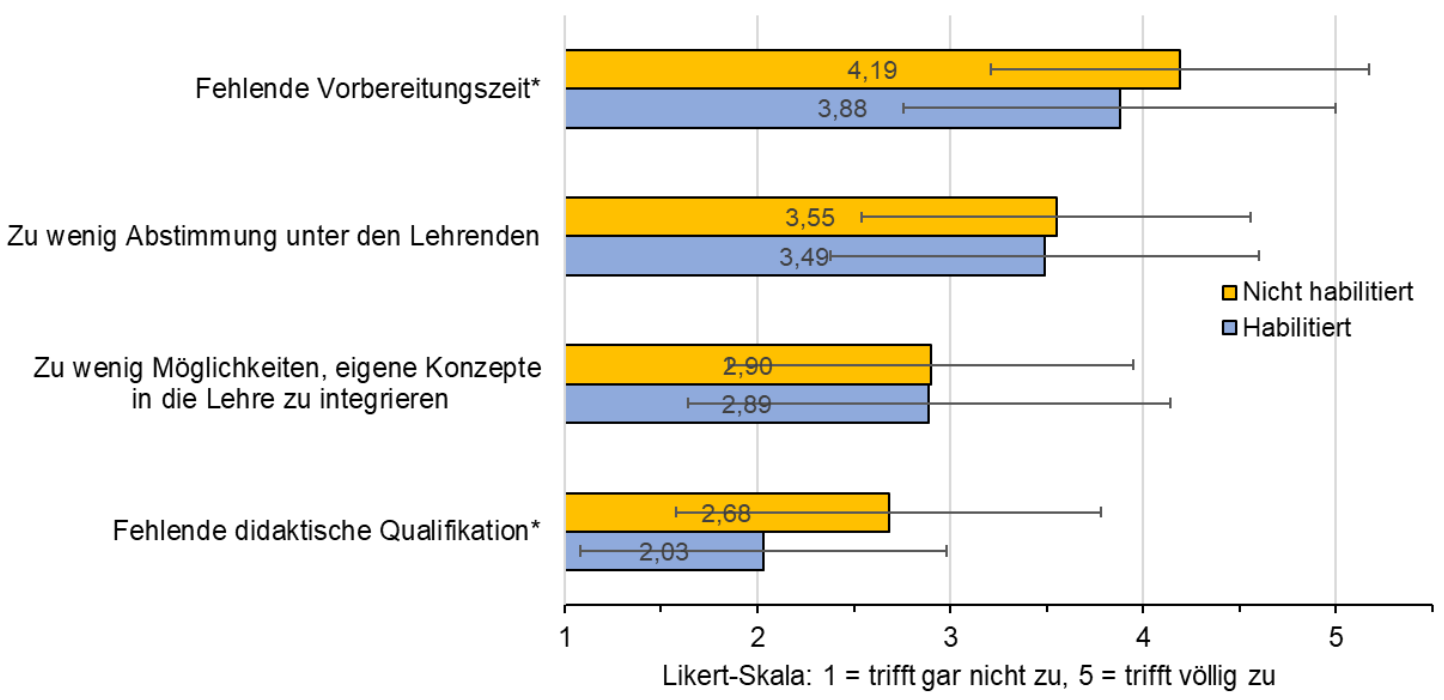

Abbildung 3: Hinderliche Faktoren. Einschätzung, inwiefern die Faktoren die Lehrenden bei der Durchführung der eigenen Lehre behindern. Die Balken repräsentieren die Mittelwerte, Fehlerbalken die Standardabweichungen (getrennt für die habilitierten und nicht habilitierten Lehrenden). ${ }^{*} p<0,05$ für den Gruppenvergleich zwischen habilitierten und nicht habilitierten Lehrenden (unabhängiger t-Test).

Die nicht habilitierten Ärzte nahmen die fehlende Vorbereitungszeit als signifikant negativer wahr als die habilitierten Ärzte $(\mathrm{t}(450,58)=-3,88 ; \mathrm{p}<0,001)$. Der Unterschied war mit Cohen's $d=0,32$ als klein einzustufen. „Fehlende didaktische Qualifikation“ wurde hingegen als weniger hinderlich einschätzt, jedoch zeigt sich hier ein Unterschied mittlerer Größe (Cohen's d = 0,65) zwischen den Gruppen. Auch in diesem Fall bewerteten die nicht Habilitierten das Hindernis als bedeutender $(\mathrm{t}(608,09)=-9,03 ; p<0,001)$. Bei der Priorisierung der angegebenen Faktoren wurde fehlende Vorbereitungszeit von $78 \%$ aller Befragten als größtes Hindernis bei der Durchführung der eigenen Lehre angesehen.

Die Ergebnisse der Frage, welche Faktoren bei der eigenen Lehre unterstützend wirken können, sind in Abbildung 4 zusammenfassend dargestellt. 
Mehr Anerkennung/Würdigung von guter Lehrleistung*

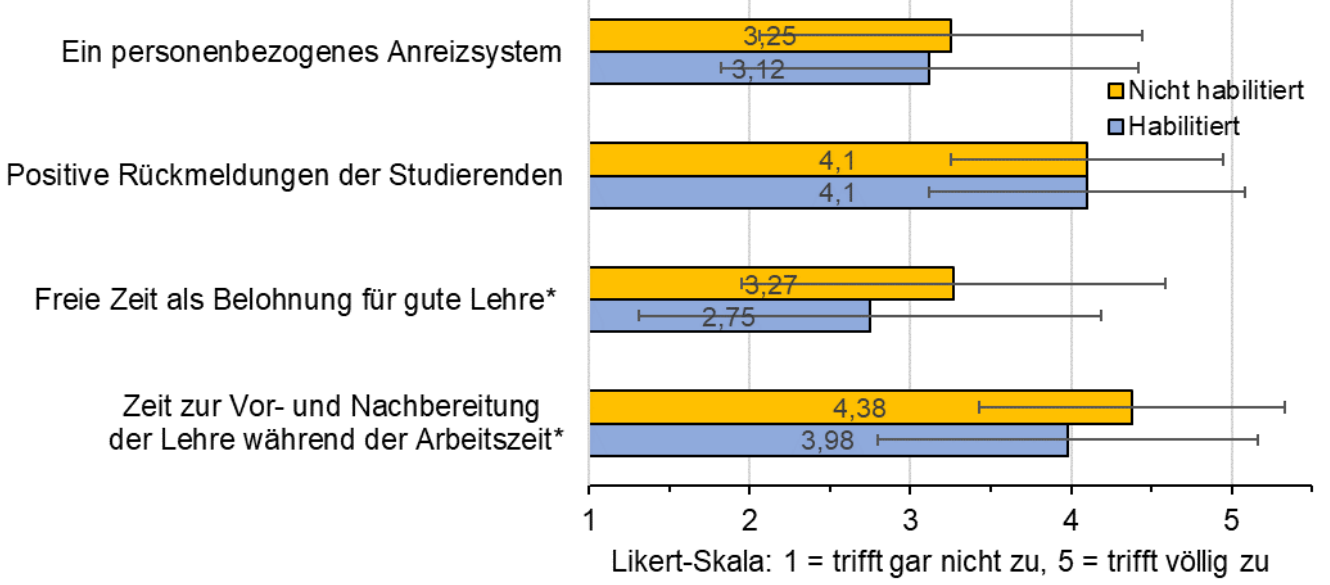

Abbildung 4: Förderliche Faktoren. Einschätzung, inwiefern die Faktoren die Lehrenden bei der Durchführung der eigenen Lehre unterstützen. Die Balken repräsentieren die Mittelwerte, Fehlerbalken die Standardabweichungen (getrennt für die habilitierten und nicht habilitierten Lehrenden). * $p<0,05$ für den Gruppenvergleich zwischen habilitierten und nicht habilitierten Lehrenden (unabhängiger t-Test).

Als am förderlichsten wurde die „Zeit zur Vor- und Nachbereitung der Lehre während der Arbeitszeit“ eingeschätzt. Hier bewerteten die nicht habilitierten Ärzte die Vor- und Nachbereitungszeit als wichtiger als ihre habilitierten Kollegen $(t(414,24)=-4,76 ; p<0,001$; Cohen's $d=0,38$ ). Beide Gruppen beurteilten gleichermaßen die „Positive Rückmeldungen der Studierenden“ als relevant. „Ein personenbezogenes Anreizsystem“ oder „Freie Zeit als Belohnung für gute Lehre" $(t(467,56)=-4,93 ; p<0,001$; Cohen's $d=0,40)$ wurde hingegen als weniger wichtig wahrgenommen. $49 \%$ der Befragten wiesen „Zeit zur Vor- und Nachbereitung der Lehre während der Arbeitszeit" die höchste Priorität unter den aufgeführten unterstützenden Faktoren zu.

Hinsichtlich der eigenen Erfahrungen mit medizindidaktischen Weiterbildungen gaben $58 \%$ der Befragten (nicht habilitiert $=48 \%$, habilitiert $=80 \% ; \chi^{2}(1, N=828)=77,72 ; \mathrm{p}<$ 0,001 ) an, bereits an solchen teilgenommen zu haben, und $78 \%$ (nicht habilitiert $=84 \%$; habilitiert $\left.=60 \% ; \chi^{2}(1, N=813)=68,59 ; p<0,001\right)$ zeigten ein generelles Interesse daran. Als Hauptgrund für die Nichtteilnahme an den Weiterbildungen wurde von $64 \%$ der Teilnehmer (nicht habilitiert $=67 \%$; habilitiert $=56 \% ; \chi^{2}(1, N=806)=9,07 ; p<0,001$ ) mangelnde Zeit genannt.

Bezogen auf die Evaluation der eigenen Lehre teilten $32 \%$ der Befragten mit, die Evaluationsergebnisse ihrer Lehrveranstaltungen regelmäßig zu erhalten (nicht habilitiert = $26 \%$, habilitiert = $44 \%$ ). $42 \%$ (nicht habilitiert = $44 \%$, habilitiert $=43 \%$ ) erhielten sie 
manchmal und $24 \%$ (nicht habilitiert = $30 \%$, habilitiert = $12 \%$;) erhielten niemals die Ergebnisse. Der $\chi^{2}$-Test war hier signifikant $\left(\chi^{2}(2, \mathrm{~N}=822)=39,54 ; \mathrm{p}<0,001\right) .60 \%$ der Teilnehmer (nicht habilitiert $=55 \%$; habilitiert $=72 \% ; \chi^{2}(1, N=764)=21,21 ; p<0,001$ ) gaben an, dass sie bereits versucht hätten, ihre Lehre basierend auf den Evaluationsergebnissen zu verbessern. $66 \%$ nutzten die Evaluationsergebnisse als Feedbackquelle und $57 \%$ (nicht habilitiert $=54 \%$, habilitiert $=63 \% ;\left(\chi^{2}(1, N=828)=4,98\right.$; $p=0,03)$ zur Verbesserung der eigenen Lehre. $47 \%$ der Befragten sagten, dass die Ergebnisse die eigene Motivation zur Lehre steigern würde, $44 \%$ sahen den Nutzen in der Qualitätssicherung (nicht habilitiert = $39 \%$; habilitiert = $54 \% ; \chi^{2}(1, N=828)=15,97 ; \mathrm{p}<$ 0,001), und lediglich $24 \%$ zogen die Daten zum Vergleich mit anderen Lehrenden heran. Bezogen auf die Form der Evaluation fanden sich keine Unterschiede zwischen den Gruppen: $75 \%$ der Befragten präferierten eine dozentenspezifische Evaluation gegenüber einer veranstaltungs- (61\%) und lernzielbezogenen (42\%) Evaluation. Lediglich $20 \%$ hielten Globalnoten für besonders sinnvoll. Bei der Frage nach dem bevorzugten Format zur Bewertung der eigenen Lehrleistung wünschten sich $66 \%$ Freitextkommentare, 56 \% fanden die Bewertung durch skalierte Items sinnvoll, $38 \%$ nannten Gruppenfeedback mit Studierenden, und weitere $33 \%$ wünschten sich Feedback durch einen Coach bzw. Trainer, wobei hier ein signifikanter Unterschied zwischen den Gruppen zu finden war (nicht habilitiert $=36 \%$, habilitiert $\left.=26 \%, \chi^{2}(1, N=832)=8,09 ; p=0,004\right)$.

\subsection{Implementierung eines neuen dozentenspezifischen Evaluationsinstruments}

Schiekirka-Schwake S, Dreiling K, Pyka K, Anders S, von Steinbüchel N, Raupach T (2018): Improving evaluation at two medical schools. Clin Teach $\underline{15}$, 314-318

In den Fokusgruppendiskussionen mit den Lehrenden sowie Studierenden wurden die gleichen relevanten Haupt- und Subthemen identifiziert. Diese sind in Tabelle 1 aufgeführt. 
Tabelle 1: Haupt- und Subthemen der Fokusgruppendiskussionen mit den Studierenden und Lehrenden

\begin{tabular}{|l|l|}
\hline \multicolumn{1}{|c|}{ Hauptthema } & \multicolumn{1}{c|}{ Subthemen } \\
\hline \multirow{2}{*}{ Das (neue) Evaluationsinstrument } & Erfassen der individuellen Lehrleistung \\
\cline { 2 - 2 } & Erfassen des studentischen Lernerfolgs \\
\hline \multirow{2}{*}{ Die Organisation } & Positives \\
\cline { 2 - 2 } & Negatives \\
\hline \multirow{3}{*}{ Verbesserungsvorschläge } & Organisation \\
\cline { 2 - 2 } & Evaluationsinstrument \\
\cline { 2 - 2 } & Form der Datensammlung \\
\hline \multirow{2}{*}{ Motivation } & Motivation der Studierenden \\
\cline { 2 - 2 } & Motivation der Lehrenden \\
\hline
\end{tabular}

\subsubsection{Ergebnisse der Fokusgruppen mit den Lehrenden}

Die befragten Lehrenden schätzten den persönlichen Nutzen des neuen Evaluationsinstruments als groß ein. Das Instrument lieferte innen wichtige Informationen über ihre individuelle Lehrleistung, die sie bisher nicht erhalten hatten. Des Weiteren ermöglichte innen das spezifische Feedback, die eigene Lehre hinsichtlich der konkret genannten Aspekte zu verbessern.

„ICh finde das total klasse, wenn man eine spezielle Personenevaluation kriegt, weil man dann ganz genau weiß, was man vielleicht besser machen kann." (Lehrender, Einzelinterview, Göttingen)

Insbesondere die Kombination der zwei Evaluationsinstrumente wurde als sinnvoll bewertet, da die Lehrenden so Informationen über die verschiedenen Dimensionen der Lehrqualität erhielten und nicht lediglich die studentische Zufriedenheit im Vordergrund stand.

„Wenn ich schon eine so konkrete Evaluation mache, dann möchte ich die Info inhaltlich kriegen: welche Punkte sind wie, welche Lernziele sind besser vermittelt worden und welche nicht." (Lehrender, Fokusgruppe, Hamburg)

Die Studienteilnehmer, die individuelle Feedbackreports erhielten, beurteilten positiv, dass sie ihre Lehrleistung im Vergleich zu den durchschnittlichen Daten der gesamten Dozentengruppe, die die gleiche Veranstaltung durchgeführt hatte, interpretieren konnten. 
weil man dann den direkten Vergleich hat zu dem, das ist halt von allen Studierenden auf alle Dozenten. Da kann man sich besser einordnen und wiederfinden." (Lehrender, Einzelinterview, Göttingen)

Sie zeigten außerdem ein großes Interesse an den Freitextkommentaren der Studierenden und lieferten kleinere Optimierungsvorschläge bzgl. der Feedbackreports. So schlugen sie beispielsweise vor, Farbkodierungen für die individuellen Ergebnisse, die über, im oder unter den durchschnittlichen Ergebnissen aller Lehrenden der gleichen Veranstaltung lagen, einzuführen.

Die Dozenten in Hamburg, die aufgrund der Entscheidung der Verantwortlichen vor Ort lediglich die aggregierten Daten erhielten, gaben an, dass sie von detaillierteren Informationen deutlich mehr profitieren würden. Auch sahen sie keine Sinnhaftigkeit darin, dass dozentenspezifische Daten erhoben wurden, sie dann aber kein individuelles Feedback erhielten.

„Bei uns war das schwierig, weil vorher gesagt wurde, es ist keine dozentenspezifische Evaluation. Wenn man sich die Fragebögen dann anguckt, dann aber sehr wohl viele Fragen über Dozenten drin sind. Ein zweiter Teil, der sehr kritisch gesehen wurde, war, dass man ja keine Rückmeldung bekommt."(Lehrender, Fokusgruppe, Hamburg)

An beiden medizinischen Fakultäten bewerteten die beteiligten Lehrenden den Implementierungsprozess des neuen Evaluationsinstruments als optimierungsbedürftig. Viele Dozenten teilten mit, dass sie die Informationen über das Projekt und den Ablauf im Vorfeld nicht erhalten hatten oder die Informationen unzureichend waren.

„Und dann bekam ich direkt von Ihnen eine Mail, dass in meiner Lehrveranstaltung evaluiert wird. Und weil ich gerade ... bestimmt 40 nicht geöffnete Mails hatte, hatte ich nur die Überschrift gelesen." (Lehrender, Fokusgruppe, Hamburg)

Die Teilnehmer waren außerdem an detaillierteren Informationen über den Fragebogen, das exakte Ziel und die Organisation des Forschungsprojekts interessiert.

„Grundsätzlich wäre es nicht schlecht, wenn zu dem [...] Projekt oder zu der Entwicklung des Fragebogens noch ein besseres Angebot für Interessierte da wäre." (Lehrender, Einzelinterview, Göttingen)

Die Dozenten nahmen die studentische Motivation zum Ausfüllen des Evaluationsinstruments verschieden wahr. Gemäß einiger Lehrenden konnte die studentische Motivation gesteigert werden, indem sie den persönlichen Nutzen der 
Evaluationsergebnisse für sich selbst erklärten und die Studierenden während der Veranstaltungszeit evaluieren ließen.

\subsubsection{Ergebnisse der Fokusgruppen mit den Studierenden}

Die Meinungen der Studierenden bezogen auf das neue Evaluationsinstrument variierten. Die meisten beurteilten den Evaluationszeitpunkt als sinnvoll. Ebenso schätzten sie das Bewerten spezifischer Aspekte der individuellen Lehrleistung als positiv ein. Insbesondere die Evaluationsitems, die sich auf konkrete Lernziele bezogen, wurden als wichtig erachtet.

„Den Vorteil, den ich bei der Evaluation sehe, ist halt, dass spezifisch die Lernziele abgerufen worden sind." (Studierender, Fokusgruppe, Hamburg)

Die Studierenden gaben an, dass sich diese auch sehr gut zur eigenen Wissensüberprüfung eignen würden. Kritisch wurde jedoch die Länge des Fragebogens und die Evaluationshäufigkeit gesehen.

„..., aber er [der Fragebogen] ist deutlich zu lang. Das schreckt viele ab. Gerade vor dem Aspekt: 5 Minuten bis zum nächsten Raum [für die nächste Veranstaltung]. “ (Studierender, Fokusgruppe, Hamburg)

Des Weiteren betonten die Studierenden, dass sie nicht in ihrer Freizeit evaluieren möchten. Bezogen auf das Erhebungsformat (online versus papierbasiert) zeigten die Studierenden keine klaren Präferenzen. Ferner gaben sie an, dass eine direkte positive Ansprache der einzelnen Lehrenden ihre Motivation zum Ausfüllen der Evaluation deutlich steigerte. Die offiziellen Einladungen durch die Projektverantwortlichen oder Modulkoordinatoren konnten dies nicht erreichen. Die Studierenden, die den (falschen) Eindruck gewonnen hatten, die Daten würden lediglich zu Forschungszwecken gesammelt werden, waren nicht bereit, weitere Evaluationsfragebögen auszufüllen.

„Die Motivation war ok, bis zu dem Punkt, bis das Gerücht eingestreut war: das wird eh nicht durchgelesen, es ist alles nur für eine Studie.” (Studierender, Fokusgruppe, Hamburg)

Sie zeigten außerdem ein großes Interesse an dem Feedback, welches aus den Evaluationsdaten für die Lehrenden generiert und welche Konsequenzen daraus gezogen wurden.

„Ich habe immer bisschen das Gefühl... klar, ich evaluiere, aber ich sehe nicht, was davon zurückkommt. Ich sehe nicht, was davon gemacht wird. Ich sehe nicht, wo die Bemühungen auch sind." (Studierender, Fokusgruppe, Hamburg) 
Insgesamt fühlten sich manche Studierende jedoch bezüglich des Implementierungsprozesses des neuen Instruments nicht ausreichend informiert.

„Ein bisschen mehr Offenlegung wäre generell nicht schlecht.“(Studierender, Fokusgruppe, Göttingen)

\subsubsection{Ergebnisse der Telefoninterviews}

Die Telefoninterviews bestätigten zum einen die Ergebnisse der Fokusgruppendiskussionen: Das persönliche und konkrete Feedback wurde von der Mehrheit der Lehrenden als positiv (63\%) bis sehr positiv (21\%) bewertet. Zum anderen wurden die veränderten Feedbackreports von dem Großteil (84\%) der Interviewten als verbessert angesehen. Insbesondere die Farbkodierungen und das Vorhandensein der Freitextkommentare wurde als sinnvoll erachtet. $30 \%$ der Befragten wiesen schon sehr gute, kaum optimierbare Evaluationsergebnisse auf. Die Hälfte der Lehrenden gab an, dass sie die eigene Lehre basierend auf den spezifischen Evaluationsergebnissen in jedem Fall optimieren möchten. Wenige Lehrende (9\%) waren sich nicht sicher oder wollten, trotz identifiziertem Optimierungsbedarf, nichts (7\%) an ihrer Lehre verändern.

\section{Diskussion}

Die vorliegenden Studien wurden durchgeführt, um die Rahmenbedingungen zur Erreichung einer hohen Lehrqualität in der medizinischen Lehre näher zu beleuchten. Ein Schwerpunkt wurde dabei auf die Herausforderungen gelegt, mit denen sich insbesondere klinisch tätige Ärzte bei der Durchführung der eigenen Lehre konfrontiert sehen. Des Weiteren wurde eruiert, welche Faktoren für diese Personengruppe hinderlich bzw. förderlich wirken und welche Rolle dabei die akademische Qualifikation (nicht habilitiert versus habilitiert) spielt.

Ein weiterer Schwerpunkt lag auf dem Aspekt Lehrevaluation. Hier wurde der Implementierungsprozess eines neuen dozentenspezifischen Evaluationsinstruments aus Sicht der beteiligten Studierenden und Lehrenden analysiert. Darüber hinaus wurde der persönliche Nutzen der individuellen Rückmeldungen, die aus den Evaluationsdaten generiert wurden, für die Lehrenden untersucht. 


\subsection{Diskussion der Ergebnisse der Fragebogenuntersuchung}

Die bundesweite Fragebogenerhebung konnte die Ergebnisse der in Göttingen durchgeführten Fokusgruppen und Pilotierungsstudie bestätigen.

Die klinisch tätigen Ärzte, die an der Fragebogenerhebung teilnahmen, wiesen ein multidimensionales Verständnis von guter Lehre auf. Diverse Aspekte der verschiedenen Dimensionen von Lehre wurden als relevant für eine hohe Lehrqualität erachtet. Bei der Aufforderung zur Priorisierung der Aspekte nannten die Lehrenden an erster Stelle einen nachhaltigen Lernerfolg. An zweiter und dritter Stelle wurden prozedurale Aspekte wie Schaffen von Begeisterung und Spaß für die Studierenden genannt. Die habilitierten und nicht habilitierten Lehrenden unterschieden sich kaum in ihren Einschätzungen.

Die Herausforderung, mit der die klinisch tätigen Lehrenden bezogen auf die eigene Lehrtätigkeit am stärksten zu kämpfen hatten, war fehlende Vorbereitungszeit für Lehre. Die nicht habilitierten Dozenten empfanden diesen Mangel als hinderlicher als ihre habilitierten Kollegen. Kongruent dazu schätzten sie eine geschützte Vor- und Nachbereitungszeit für Lehre in der regulären Arbeitszeit als etwas förderlicher ein als die habilitierten Lehrenden. Bezogen auf didaktische Fortbildungen zeigten die meisten Befragten zwar ein generelles Interesse, das Fehlen didaktischer Qualifikationen wurde jedoch, insbesondere für die habilitierten Lehrenden, als kaum bis gar nicht limitierend wahrgenommen. Als Hauptgrund für die Nichtteilnahme an didaktischen Fortbildungen wurde konsistent mangelnde Zeit genannt.

Zusätzlich zur gesicherten Zeit für Lehre empfanden beide Gruppen gleichermaßen positives studentisches Feedback als besonders unterstützend bzw. motivierend. Die Würdigung von guter Lehre war für die nicht habilitierten Dozenten bedeutender als für die habilitierten. Monetäre Anreize wurden lediglich als teilweise wichtig eingeschätzt.

Drei Viertel der Befragten wünschten sich eine dozentenspezifische Evaluation. In der Realität erhielten jedoch nicht alle Lehrenden ihre Evaluationsergebnisse. Und noch weniger erhielten die Ergebnisse tatsächlich regelmäßig. Insbesondere den nicht habilitierten Dozenten fehlte es an regelmäßigem Feedback. Dies scheint besonders vor dem Hintergrund, dass positives studentisches Feedback als einer der beiden wichtigsten unterstützenden Faktoren eingeschätzt wurde, problematisch. Lagen den Lehrenden ihre Evaluationsdaten vor, wurden diese auch meist zur Optimierung der eigenen Lehrleistung herangezogen.

Verbesserungswürdige Lehrbedingungen für Lehrende in der Medizin konnten bereits in verschiedenen Studien identifiziert werden (Arabshahi et al. 2015; Barrett und Scott 2014; Hoffman und Donaldson 2004; Huwendiek et al. 2013; Huwendiek et al. 2010; Müller-Hilke 2010; Peadon et al. 2010; Sabel und Archer 2014; Seabrook 2003). Es scheint ein 
internationales, bisher ungelöstes Problem, auch wenn dieses schon vor Jahren bzw. Jahrzehnten erkannt wurde (Abrahamson 1996; Seabrook 2003). Zwar sieht Müller-Hilke (2010) den Stellenwert der Lehre im Umbruch, bedeutende flächendeckende Veränderungen konnten bis dato jedoch nicht identifiziert werden. Die Ergebnisse der vorliegenden deutschlandweiten Untersuchung bestätigen diese Problematik für die teilnehmenden medizinischen Fakultäten. Wie in einem Großteil der bisherigen qualitativen Forschungsarbeiten, die sich auf die Personengruppe der klinisch tätigen Ärzte bezogen (Barrett und Scott 2014; Knight und Bligh 2006; Peadon et al. 2010; Seabrook 2003), gezeigt wurde, konnte auch in der vorliegenden Untersuchung fehlende Zeit als bedeutendster limitierender Faktor zur Erreichung einer hohen Lehrqualität identifiziert werden. Fehlende didaktische Qualifikation wurde von den befragten Ärzten hingegen als kaum hinderlich wahrgenommen. Als eine Erklärung für das letztgenannte Ergebnis scheint augenscheinlich, dass $80 \%$ der befragten habilitierten Ärzte bereits an didaktischen Fortbildungen teilgenommen hatten. Von den nicht habilitierten waren es $48 \%$. Darüber hinaus flossen möglicherweise selbstwertdienliche Verzerrungen mit in die Einschätzung ein, so dass sich die Lehrenden mutmaßlich keine mangelnde didaktische Qualifikation zuschreiben mochten. Diese These stützt unter anderem die Studie von Cross (1977), in der $94 \%$ der befragten Lehrenden die eigenen Vortragsfertigkeiten im Vergleich zu den Vortragsfertigkeiten der Kollegen als überdurchschnittlich einschätzten. In den vorhergehenden Studien spielte selbst wahrgenommene mangelnde Kompetenz als limitierender Faktor ebenfalls meist eine eher nachrangige Rolle, auch wenn in der Regel ein zusätzlicher Bedarf an didaktischen Weiterbildungen identifiziert wurde (Huwendiek et al. 2013; Huwendiek et al. 2010; Peadon et al. 2010).

Die Ergebnisse früherer Forschungsarbeiten hinsichtlich monetärer Anreize für Engagement in der Lehre sind divergent. Teilweise wurden finanzielle Belohnungen als wenig bedeutend eingeschätzt (Dybowski und Harendza 2014; Müller-Hilke 2010), andere Studien eruierten Gegensätzliches (Hofer et al. 2008; Huwendiek et al. 2013; Huwendiek et al. 2010). Die Daten der Fragebogenuntersuchung unterstützen die Erkenntnisse der Studien von Müller-Hilke (2010) sowie Dybowski und Harendza (2014). Müller-Hilke (2010) sieht als eine mögliche Erklärung für die nachrangige Bewertung von monetären Anreizen den Selektionsbias. So nehmen an solchen Befragungen vermutlich die Personen teil, die der Lehre generell einen höheren Stellenwert zuschreiben. Im Einklang mit der Studie von Müller-Hilke (2010) steht ebenso das Ergebnis der bedeutenden motivierenden Wirkung von positiven studentischen Rückmeldungen für die Lehrenden.

An dieser Stelle sei außerdem darauf hingewiesen, dass die befragten Lehrenden ein ähnlich breites Verständnis von guter Lehre, mit vergleichbar gesetzten Schwerpunkten, aufwiesen wie Medizinstudierende, die im Rahmen einer qualitativen Studie dazu befragt 
wurden (Schiekirka et al. 2012). Diese Kongruenz bezüglich des Konstrukts von guter Lehre bildet die wichtige Voraussetzung, dass die vom Lehrenden angestrebte hohe Lehrqualität auch von den Studierenden als solche wertgeschätzt wird und so zu positivem Feedback als motivierenden Faktor führen kann.

In den Studien von Huwendiek und Kollegen (Huwendiek et al. 2013; Huwendiek et al. 2010) wurde das Fehlen akademischer Wertschätzung von den Lehrenden als größte Herausforderung benannt. In der vorliegenden Arbeit war der Wunsch danach weniger präsent, wenn auch von den nicht habilitierten Lehrenden größer als von den habilitierten. Dies mag darauf zurückzuführen sein, dass Huwendiek und Kollegen (Huwendiek et al. 2013; Huwendiek et al. 2010) ihre Stichproben nicht auf klinisch tätige Ärzte begrenzten. Durch die Dreifachbelastung von Patientenversorgung, Forschung und Lehre steht fehlende Zeit für die Befragten möglicherweise so stark im Vordergrund, dass andere Faktoren an Bedeutung verlieren.

Es ist bedenklich, dass - obwohl Lehre ein fester Bestandteil der Aufgaben von Ärzten an Universitätskliniken ist - die Lehrenden so wenige unterstützende Maßnahmen für die Durchführung der eigenen Lehre erfahren. Die Daten lassen darauf schließen, dass zumindest bei den teilnehmenden medizinischen Fakultäten Optimierungsbedarf herrscht. Betrachtet man die vergleichenden Daten der nicht habilitierten sowie habilitierten Ärzte, lässt sich aus diesen schlussfolgern, dass insbesondere in den ersten Jahren nach der Approbation die klinisch tätigen Lehrenden von unterstützenden Maßnahmen profitieren würden. Die habilitierten Ärzte scheinen sich aufgrund ihrer Erfahrung, höherer didaktischer Qualifikationen und dem größeren Maß an Selbstbestimmtheit besser mit den gegebenen Lehrbedingungen arrangieren zu können.

Lösungsansätze für diese Problematiken wurden bereits mehrfach diskutiert (DaRosa et al. 2011; Gerrity et al. 1997; Schormair et al. 1992). Sicherlich darf die Qualität der Patientenversorgung nicht unter den Veränderungen leiden, da dies schwerwiegende Folgen nach sich ziehen könnte. Dennoch sollten die Verantwortlichen an den Universitätskliniken ihre ärztlichen Mitarbeiter ebenso unterstützen, bestärken und motivieren, gute Lehrleistungen zu erbringen. Vor allem geschützte Zeit für die Durchführung der Lehre sowie deren Vor- und Nachbereitung scheint zu fehlen.

Um dies erreichen zu können, könnte die Einstellung von zusätzlichem Personal unterstützend wirken. Wünschenswert wäre außerdem das Schaffen von Arbeitsplätzen, deren Fokus verstärkt auf der Lehre liegt („clinician educators“). Solche neuen Mitarbeiter könnten zum Beispiel nicht nur selbst lehren, sondern auch Ärzte, die sich am Beginn ihrer beruflichen Laufbahn befinden, in ihren Lehrtätigkeiten unterstützen und supervidieren. Eine weitere ökonomischere Strategie könnte sein, vermehrt Medizinstudierende in 
Lehraktivitäten einzubinden. Ferner könnte im Einklang mit den interprofessionellen Entwicklungen beispielsweise Pflegepersonal stärker in die Lehre integriert werden.

Von Vorteil wäre es außerdem, wenn die Patientenversorgung, Forschung sowie Lehre an medizinischen Fakultäten nicht als konkurrierende Aufgabenfelder wahrgenommen würden, sondern alle Bereiche gleichermaßen Raum und akademische Anerkennung erhielten. So könnten beispielsweise Abteilungen zusätzliche finanzielle Mittel dafür erhalten, wenn sie ihre Mitarbeiter an didaktischen Weiterbildungen teilnehmen ließen. Darüber hinaus könnten vergebene Lehrpreise eine positive Lehrkultur unterstützen.

Da sich ferner gezeigt hat, dass positives studentisches Feedback einen bedeutenden motivierenden Faktor darstellt und Evaluationsergebnisse zur Optimierung der eigenen Lehrleistung herangezogen werden, stellt die regelmäßige Bereitstellung individueller Evaluationsergebnisse eine weitere wichtige Maßnahme dar. Hierbei ist der Einsatz von validen und reliablen Instrumenten grundlegend. Des Weiteren sollte vorab bedacht werden, wie sinnvoll mit kritischen Rückmeldungen umgegangen werden könnte, da diese einen negativen Einfluss auf die Motivation und das Wohlbefinden der Lehrenden ausüben können (Dybowski und Harendza 2014).

Bei der Implementierung von Maßnahmen - wie in dieser Arbeit beschrieben - ist das Einbeziehen der Erkenntnisse aus dem Veränderungsmanagement entscheidend (Anderson und Ackermann Anderson 2001; Bland et al. 2000; Gale und Grant 1997). Auch sollten die Veränderungen wissenschaftlich begleitet und evaluiert werden. So schreiben Dybrowksi und Harendza (2014), dass bereits einige Maßnahmen, wie zum Beispiel die Einführung von Lehrpreisen, umgesetzt wurden, jedoch nicht zu den gewünschten positiven und motivierenden Veränderungen geführt hätten. Folglich besteht Forschungsbedarf im Hinblick auf die Wirksamkeit der Maßnahmen und welche weiteren Einflussfaktoren hierbei eine Rolle spielen. Des Weiteren sollten zusätzliche Maßnahmen identifiziert werden.

Es lassen sich verschiedene Stärken und Limitationen der ersten hier dargestellten Studie identifizieren. Im Gegensatz zu vorherigen Arbeiten in dem Bereich wurde sich in der vorliegenden Studie auf eine sehr spezifische Gruppe von Lehrenden fokussiert. Dies schränkt zwar die Generalisierbarkeit der Daten auf andere Berufsgruppen, die in der medizinischen Lehre tätig sind, ein, hilft aber zu verstehen, warum insbesondere klinisch tätige Ärzte bisweilen Schwierigkeiten haben, gute Lehre durchzuführen, und welche Maßnahmen helfen könnten, die Situation für sie zu verbessern. Eine besondere Stärke der Fragebogenuntersuchung ist die Stichprobengröße und -diversität: Es konnten Daten von über 800 klinisch tätigen Ärzten verschiedener medizinischer Fakultäten aus acht Bundesländern in die Analyse eingeschlossen werden. Die Interpretation der Ergebnisse der Studie ist dahingehend limitiert, dass lediglich subjektive Daten erhoben wurden. Entsprechend beruhen die Resultate und Unterschiede zwischen den Gruppen 
ausschließlich auf der Wahrnehmung der Befragten. Des Weiteren konnten die meisten eingeschlossenen Fakultäten keine genauen Auskünfte darüber geben, wie viele der klinisch tätigen Ärzte tatsächlich in die Lehre involviert sind. Aufgrund dessen ließ sich kein Rücklauf berechnen oder eine Poweranalyse durchführen. Ferner sind alle statistischen Vergleiche explorativer Natur. Abschließend ist auf die Selbstselektion der teilnehmenden Fakultäten hinzuweisen. Möglicherweise ist die Stichprobe infolgedessen nicht repräsentativ für alle medizinischen Fakultäten in Deutschland.

\subsection{Diskussion der Ergebnisse der Implementierungsstudie}

Im folgenden Abschnitt werden die Ergebnisse der zweiten hier kumulativ dargestellten Arbeit diskutiert sowie deren Stärken und Schwächen erläutert.

Die evaluierten Lehrenden nahmen den neu eingesetzten Fragebogen als positiv wahr. Sie schätzten vor allem das individuelle Feedback, welches durch das Instrument bezogen auf die verschiedenen Dimensionen der Lehre generiert werden konnte. Auch bewerteten sie den Vergleich mit den durchschnittlichen Daten der gesamten Dozentengruppe als hilfreich. In den Telefoninterviews gaben viele Befragte an, die Daten zur Verbesserung der eigenen Lehre heranzuziehen. Des Weiteren wurde die Wichtigkeit der Freitextkommentare betont. Die Studierenden empfanden die spezifischen, insbesondere die inhaltsbezogenen, Aspekte, die mittels des Instruments erfasst wurden, ebenfalls als sinnvoll. Sie sahen jedoch die Länge des Fragebogens als kritisch an und wünschten sich, während der Veranstaltungszeit evaluieren zu können. Außerdem baten sie um Informationen bezüglich des Feedbacks, welches die Lehrenden erhielten, und wollten über eventuelle Maßnahmen, die basierend auf den Evaluationsergebnissen ergriffen wurden, informiert werden.

Der Implementierungsprozess des neuen Instruments wurde hingegen sowohl von den Lehrenden als auch den Studierenden als optimierungsbedürttig erlebt. Beide Personengruppen versäumten teilweise essentielle Informationen oder empfanden die bereitgestellten Informationen als unzureichend, obwohl diese durch die Projektverantwortlichen vor und während des Forschungsvorhabens mehrfach an die Lehrenden und Studierenden herangetragen wurden.

Die Ergebnisse der vorliegenden Studie bekräftigen die Daten der oben beschriebenen bundesweiten Fragebogenuntersuchung sowie der qualitativen Studie von Schiekirka et al. (2012): Sowohl die Studierenden als auch die Lehrenden verstanden gute Lehre als ein multidimensionales Konstrukt. Beide Personengruppen bewerteten positiv, dass sich das neue Evaluationsinstrument auf ein solch breites Konstrukt bezieht. Ferner steht der Wunsch der Lehrenden nach individuellem Feedback sowie die Angabe, dass dieses zur 
Verbesserung der Lehrleistung herangezogen werde, im Einklang mit den Ergebnissen der quantitativen Untersuchung, die zuvor geschildert wurde. Aburawi et al. (2014) fanden ähnliches bei einer Befragung von Lehrenden einer medizinischen Fakultät in den Vereinigten Arabischen Emiraten. Inwiefern diese Veränderungen in der Praxis umgesetzt und für die Studierenden ersichtlich werden, bleibt jedoch unklar. Wibbecke et al. (2015) betonen, dass das bloße Bereitstellen studentischer Evaluationsergebnisse wenig adäquate Verbesserungsprozesse nach sich ziehe. Die Autoren schlagen hingegen eine Kombination von inhaltsanalytisch aufbereiteten studentischen Lehrevaluationsdaten sowie Feedback durch professionelle Lehrberater vor. So könne auch vermieden werden, dass negatives, unsachlich formuliertes Feedback durch Studierende das Wohlbefinden der Lehrenden bedeutend beeinträchtigt (Dybowski und Harendza 2014). Im Rahmen dessen ist jedoch interessant, dass die Lehrenden in der vorliegenden Untersuchung insbesondere ein großes Interesse an den Freitextkommentaren zeigten, obwohl die Kommentare lediglich Einzelmeinungen darstellen können und die Studierenden diese in der vorliegenden Untersuchung nicht immer neutral formulierten.

Um die Akzeptanz und Motivation für regelmäßige Evaluationen auf Seiten der Studierenden zu erhöhen, scheint es wesentlich, diese über Sinn und Zweck der Lehrevaluation sowie über die Konsequenzen, die basierend auf ihren Evaluationsdaten gezogen werden, zu informieren (Aburawi et al. 2014; Schiekirka et al. 2012). Darüber hinaus ist geschützte Zeit zum Evaluieren während der Lehrveranstaltungen grundlegend. Des Weiteren wünschten sowohl die Lehrenden als auch die Studierenden präzisere Informationen über das neu eingesetzte Instrument. Sie wollten detailliert über den theoretischen Hintergrund, die Qualitätsmerkmale sowie Gütekriterien in Kenntnis gesetzt werden. Es scheint folglich nicht auszureichen, den in den Veränderungsprozess involvierten Parteien sehr komprimierte Informationen zukommen zu lassen. Diese Ergebnisse stehen im Einklang mit Studien des Veränderungsmanagements, die die Wichtigkeit ausgeprägter Kommunikation sowie regelmäßigen Informationsaustausch mit den involvierten Personen im Veränderungsprozess betonen, um ein Klima der Kooperation zu schaffen (Bland et al. 2000; Dannefer et al. 1998; Gale und Grant 1997; van den Heuvel et al. 2014). Dannefer und Kollegen (1998) sprechen im Rahmen dessen sogar von "geplanter Redundanz" und bezeichnen das direkte, interaktive Gespräch mit den Beteiligten als den idealen Informationsweg. Auch unterstützt der Wunsch der involvierten Personen nach umfassendem theoretischen Hintergrundwissen sowie Informationen über Gütekriterien des Instruments die von Gale und Grant (1997) aufgestellte These, dass in der Medizin ein großer Wert auf Wissenschaftlichkeit und rationale Outcome-Variablen gelegt werde. Ein weiterer nicht zu verachtender positiver Effekt, der durch die ausführliche 
Aufklärung über das neue Fragebogeninstrument erreicht werden kann, ist die Reduktion des möglichen Einflusses von Biasfaktoren (Albanese 2000; Billings-Gagliardi et al. 2004). Wenngleich die Ergebnisse darauf hinweisen, dass die Studierenden und Lehrenden ein offenbar großes Interesse an detaillierten Informationen bezüglich des Forschungsvorhabens aufwiesen, wurden sowohl von den Studierenden als auch den Lehrenden wichtige Informationen nicht wahrgenommen, obwohl diese mehrfach an beide Parteien herangetragen wurden. Mögliche Gründe dafür könnten sowohl zeitliche Überlastungen auf Seiten der Studierenden und Lehrenden sein, als auch der mutmaßlich geringe Stellenwert solcher Forschungsprojekte im Alltag der medizinischen universitären Medizin. Des Weiteren waren die gewählten Kommunikationswege möglicherweise nicht ideal, auch wenn die Informationen auf verschiedene Weise übermittelt wurden.

Lösungsansätze könnten sein, die Informationen von höherer Stelle (z. B. dem Dekanat) verbreiten zu lassen, vermehrt das direkte Gespräch mit den Beteiligten zu suchen sowie mehrfach zu überprüfen, ob alle Personen die notwendigen Informationen erhalten und verstanden haben. Zusätzlich dazu könnte man alle beteiligten Personengruppen von Beginn an mit in das Forschungsvorhaben einbinden und ausreichend Raum für Nachfragen und Diskussion einräumen. Abschließend ist noch die Möglichkeit des Einsatzes von Anreizen wie beispielsweise die Verlosung von Gutscheinen zu nennen. Eine besondere Stärke der zweiten hier kumulativ dargestellten Arbeit ist, dass Daten sowohl von Lehrenden als auch Studierenden, die von der Implementierung des neuen Instruments betroffen waren, gewonnen werden konnten. Des Weiteren wurden Daten von zwei medizinischen Fakultäten mit einbezogen. Ferner war die Stichprobe für diese Form der qualitativen Studie relativ groß. Als Limitation lässt sich benennen, dass durch die Freiwilligkeit der Teilnahme negativere Perspektiven und Meinungen von weniger motivierten Personen unterrepräsentiert sein könnten. Darüber hinaus konnten die Daten der Telefoninterviews nicht aufgenommen und wörtlich transkribiert werden.

\section{Schlussfolgerungen}

In der vorliegenden Arbeit konnte gezeigt werden, dass die Bedingungen für die Durchführung von guter Lehre in der universitären Medizin, insbesondere für klinisch tätige Ärzte zu Beginn ihrer akademischen Karriere, optimierungsbedürftig sind. Die Etablierung einer Institutionskultur, bei der Patientenversorgung, Forschungsaktivitäten sowie die Erbringung von Lehre nicht als konkurrierende Aufgabenfelder, sondern als gleichermaßen wichtig wahrgenommen werden, ist eine grundlegende Voraussetzung, um die in der vorliegenden Studie identifizierten Hürden zu überwinden. 
Des Weiteren weisen die Daten darauf hin, dass dozentenspezifische Lehrevaluation einen wichtigen und förderlichen Faktor bei der Erbringung guter Lehrleistung darstellt. Wünschenswert wäre die feste Implementierung von individuellen Evaluationsinstrumenten, die die verschiedenen Dimensionen der Lehre abbilden. Hierbei sind Feedbackschleifen essentiell, nicht nur um die Qualitätssicherung zur garantieren, sondern auch um die Akzeptanz auf Seiten der Studierenden und Lehrenden zu erhöhen. Bei Veränderungsprozessen, wie der Implementierung eines neuen Evaluationsinstruments, scheint die wiederholte und ausführliche Informationsdarstellung im Idealfalle von höherer Stelle - wesentlich für den Erfolg. Als optimaler Kommunikationsweg hat sich dabei das direkte interaktive Gespräch erwiesen. 


\section{Zusammenfassung}

Hintergrund: Die studentische Lehre in der universitären Medizin muss als ein elementarer Baustein in der Ausbildung der zukünftigen Ärzte hohen Ansprüchen genügen. Ein wesentliches Instrument zur Sicherung und Optimierung der Lehrqualität stellt hierbei die studentische Evaluation dar. Leider sind die Rahmenbedingungen zur Erreichung einer hohen Lehrqualität nicht immer gegeben. Insbesondere für klinisch tätige Ärzte bedeutet die Dreifachbelastung aus Patientenversorgung, wissenschaftlichem Engagement sowie der Durchführung von Lehre häufig eine große Herausforderung.

Ziel: Ziel der vorliegenden Arbeit war es, die Rahmenbedingungen zur Erreichung einer hohen Lehrqualität in der medizinischen Lehre $\mathrm{zu}$ eruieren sowie den Implementierungsprozess eines neuen dozentenspezifischen Evaluationsfragebogens näher zu untersuchen.

Methode: Mittels eines onlinebasierten Fragebogens wurden an neun deutschen medizinischen Fakultäten quantitative Daten erhoben. Darüber hinaus fanden an zwei medizinischen Fakultäten in Deutschland Fokusgruppendiskussionen, Telefon- sowie Einzelinterviews statt.

Ergebnisse: Im Rahmen der quantitativen Datenerhebung konnten 833 Datensätze von klinisch tätigen Lehrenden zur Analyse herangezogen werden. Des Weiteren wurden vier Fokusgruppen $(n=16)$, drei Einzel- sowie 43 Telefoninterviews mit medizinischen Lehrenden durchgeführt. Mit Medizinstudierenden fanden fünf Fokusgruppen ( $n=33)$ statt. Mangelnde geschützte Zeit für Lehre wurde als stärkster limitierender Faktor bei der Durchführung der eigenen Lehrtätigkeit wahrgenommen. Insbesondere die nicht habilitierten Ärzte erlebten diesen Mangel als hinderlicher. Kongruent dazu wurde geschützte Zeit für die Vor- und Nachbereitung von Lehre als förderlichster Faktor eingeschätzt. Dozentenspezifische Lehrevaluation bzw. individuelles Feedback wurde von den Befragten ebenfalls als ein bedeutender förderlicher Faktor bei der Erbringung guter Lehrleistung wahrgenommen. Den Implementierungsprozess des neuen Evaluationsinstruments empfanden sowohl die Studierenden als auch die Lehrenden als verbesserungswürdig.

Schlussfolgerung: Die Bedingungen für die Durchführung von guter Lehre in der universitären Medizin, insbesondere für Ärzte zu Beginn ihrer akademischen Karriere, sind optimierungsbedürftig. Die Etablierung einer Institutionskultur, bei der Patientenversorgung, Forschungsaktivitäten sowie die Erbringung von Lehre als gleichermaßen wichtig wahrgenommen werden, ist eine grundlegende Voraussetzung, um die in der vorliegenden Studie identifizierten Hürden zu überwinden. Wünschenswert wäre außerdem die feste Implementierung von individuellen Evaluationsinstrumenten und Feedbackschleifen. 


\section{Literaturverzeichnis}

Abrahamson S (1996): Time to return medical schools to their primary purpose: education. Acad Med 1ㅡ, 343-347

Aburawi E, McLean M, Shaban S (2014): Evaluation of Faculty: Are medical students and faculty on the same page? Sultan Qaboos Univ Med J $\underline{14}$, e361-e368

Al-Haddad S, Kotnour T (2015): Integrating the organizational change literature: a model for successful change. JOCM $\underline{28}, 234-262$

Albanese M (2000): Challenges in using rater judgements in medical education. J Eval Clin Pract $\underline{6}, 305-319$

Albanese M, Prucha C, Barnet JH, Gjerde CL (1997): The effect of right or left placement of the positive response on Likert-type scales used by medical students for rating instruction. Acad Med $\underline{72}, 627-630$

Aleamoni LM (1999): Student Rating Myths Versus Research Facts from 1924 to 1998. J Person Eval Educ 13, 153-166

Anderson D, Ackermann Anderson LS: Beyond Change Management: Advanced Strategies for Today's Transformational Leaders. Jossey-Bass/Pfeiffer, San Francisco 2001

Arabshahi KS, Haghani F, Bigdeli S, Omid A, Adibi P (2015): Challenges of the ward round teaching based on the experiences of medical clinical teachers. J Res Med Sci 므, 273-280

Bamford DR, Forrester PL (2003): Managing planned and emergent change within an operations management environment. IJOPM $\underline{23}, 546-564$

Barrett J, Scott K (2014): Pedagogical and professional compromises by medical teachers in hospitals. Clin Teach 11, 340-344

Beer M, Nohria N (2000): Cracking the code of change. HBR $\underline{78}, 133-141$

Berger U, Schleußner C (2003): Hängen Ergebnisse einer Lehrveranstaltungs-Evaluation von der Häufigkeit des Veranstaltungsbesuches ab? Z Päd Psychol 17, 125-131

Berger U, Schleußner C, Strauß B (2003): Umfassende Lehrevaluation in der Medizin - eine Aufgabe für die psychosozialen Fächer? Psychother Psych Med $\underline{53}$, 71-78

Berk RA (2013): Top five flashpoints in the assessment of teaching effectiveness. Med Teach $\underline{35}, 15-26$ 
Billings-Gagliardi S, Barrett SV, Mazor KM (2004): Interpreting course evaluation results: insights from thinkaloud interviews with medical students. Med Educ $\underline{38}, 1061-1070$

Bland CJ, Starnaman S, Wersal L, Moorehead-Rosenberg L, Zonia S, Henry R (2000): Curricular change in medical schools: how to succeed. Acad Med $\underline{75}, 575-594$

Burnes B (2004): Emergent change and planned change - competitors or allies?: The case of $X Y Z$ construction International. Int J Oper Prod Manag 24, 886-902

Burnes B, Jackson P (2011): Success and Failure In Organizational Change: An Exploration of the Role of Values. JCM $\underline{11}, 133-162$

By RT (2005): Organisational Change Management: A Citical Review. JCM $\underline{5}$, 369-380

Cross KP (1977): Not can, but will college teaching be improved? New Dir Higher Educ $\underline{1977}, 1-15$

Dannefer EF, Johnston MA, Krackov SK (1998): Communication and the process of educational change. Acad Med $\underline{73}, 16-23$

DaRosa DA, Skeff K, Friedland JA, Coburn M, Cox S, Pollart S, O'Connell M, Smith S (2011): Barriers to effective teaching. Acad Med $\underline{86}, 453-459$

Dimitroff A, Davis WK (1996): Content analysis of research in undergraduate medical education. Acad Med $\underline{71}, 60-67$

Dreiling K, Montano D, Poinstingl H, Muller T, Schiekirka-Schwake S, Anders S, von Steinbuchel N, Raupach T (2017): Evaluation in undergraduate medical education: Conceptualizing and validating a novel questionnaire for assessing the quality of bedside teaching. Med Teach $\underline{39}$, 820-827

Dybowski C, Harendza S (2014): "Teaching Is Like Nightshifts ...": A Focus Group Study on the Teaching Motivations of Clinicians. Teach Learn Med 26, 393-400

Dybowski C, Sehner S, Harendza S (2017): Influence of motivation, self-efficacy and situational factors on the teaching quality of clinical educators. BMC Med Educ 17, 84-84

Elzubeir M, Rizk D (2002): Evaluating the quality of teaching in medical education: are we using the evidence for both formative and summative purposes? Med Teach $\underline{24}$, 313-319

Gale R, Grant J (1997): AMEE Medical Education Guide No 10: managing change in a medical context: guidelines for action. Med Teach $\underline{19}, 239-249$ 
Gerrity MS, Pathman DE, Linzer M, Steiner BD, Winterbottom LM, Sharp MC, Skochelak SE (1997): Career satisfaction and clinician-educators. The rewards and challenges of teaching. The Society of General Internal Medicine Career Satisfaction Study Group. J Gen Intern Med 12 (Suppl 2), 90-97

Gibson KA, Boyle P, Black DA, Cunningham M, Grimm MC, McNeil HP (2008): Enhancing Evaluation in an Undergraduate Medical Education Program. Acad Med $\underline{83}, 787-793$

Griffin BW (2001): Instructor Reputation and Student Ratings of Instruction. Contemp Educ Psychol 26, 534-552

Hartley S, Macfarlane F, Gantley M, Murray E (1999): Influence on general practitioners of teaching undergraduates: qualitative study of London general practitioner teachers. BMJ $\underline{319}, 1168-1171$

Hofer M, Pieper M, Sadlo M, Reipen J, Heussen N (2008): Leistungsorientierte Mittelvergabe (LoM) in der Medizinischen Lehre. DMW 농, 615-620

Hoffman KG, Donaldson JF (2004): Contextual tensions of the clinical environment and their influence on teaching and learning. Med Educ $\underline{38}$, 448-454

Huwendiek S, Hahn EG, Tönshoff B, Nikendei C (2013): Herausforderungen Lehrender in der Medizin: Ergebnisse einer Umfrage unter Mitgliedern der Gesellschaft für Medizinische Ausbildung. GMS J Med Educ $\underline{30}$, Doc38

Huwendiek S, Mennin S, Dern P, Ben-David MF, Van Der Vleuten C, Tonshoff B, Nikendei C (2010): Expertise, needs and challenges of medical educators: Results of an international web survey. Med Teach 32, 912-918

Iblher P, Zupanic M, Härtel C, Heinze H, Schmucker P, Fischer MA (2011): Der Fragebogen "SFDP26-German": Ein verlässliches Instrument zur Evaluation des klinischen Unterrichts? GMS J Med Educ 요, Doc30

Knight LV, Bligh J (2006): Physicians' Perceptions of Clinical Teaching: A Qualitative Analysis in the Context of Change. Adv Health Sci Educ Theory Pract 11, 221-234

Kogan JR, Shea JA (2007): Course evaluation in medical education. Teach Teach Educ $\underline{23}$, 251-264

Marsh HW (1983): Multidimensional ratings of teaching effectiveness by students from different academic settings and their relation to student/course/instructor characteristics. $J$ Educ Psychol $\underline{75}$, 150-166 
Marsh HW, Roche LA (1997): Making students' evaluations of teaching effectiveness effective: The critical issues of validity, bias, and utility. Am Psychol $\underline{52,1187-1197}$

Mayring P: Qualitative Inhaltsanalyse - Grundlagen und Techniken. 11. Auflage; Beltz Verlag, Weinheim und Basel 2010

Michel A, Todnem By R, Burnes B (2013): The limitations of dispositional resistance in relation to organizational change. Manage Decis $\underline{51}, 761-780$

Müller-Hilke B (2010): "Ruhm und Ehre" oder LOM für Lehre? - eine qualitative Analyse von Anreizverfahren für gute Lehre an Medizinischen Fakultäten in Deutschland. GMS J Med Educ 27, Doc43

Müller T, Montano D, Poinstingl H, Dreiling K, Schiekirka-Schwake S, Anders S, Raupach T, von Steinbüchel N (2017): Evaluation of large-group lectures in medicine - development of the SETMED-L (Student Evaluation of Teaching in MEDical Lectures) questionnaire. BMC Med Educ 17, 137

Naftulin DH, Ware JE, Donnelly FA (1973): The Doctor Fox Lecture: A Paradigm of Educational Seduction. J Med Educ 4요, 630-635

Peadon E, Caldwell PH, Oldmeadow W (2010): 'I enjoy teaching but...': Paediatricians' attitudes to teaching medical students and junior doctors. J Paediatr Child Health $\underline{46}, 647-$ 652

Raupach T, Münscher C, Beissbarth T, Burckhardt G, Pukrop T (2011): Towards outcomebased programme evaluation: Using student comparative self-assessments to determine teaching effectiveness. Med Teach $\underline{33}$, e446-e453

Raupach T, Schiekirka S, Munscher C, Beissbarth T, Himmel W, Burckhardt G, Pukrop T (2012): Piloting an outcome-based programme evaluation tool in undergraduate medical education. GMS J Med Educ 29 , Doc44

Rost DH: Interpretation und Bewertung pädagogisch-psychologischer Studien. 2. vollständig überarb. u. erw. Auflage 2007; Beltz Verlag, Weinheim und Basel 2005

Rutter H, Herzberg J, Paice E (2002): Stress in doctors and dentists who teach. Med Educ 36, 543-549

Sabel E, Archer J (2014): "Medical education is the ugly duckling of the medical world" and other challenges to medical educators' identity construction: a qualitative study. Acad Med 89, 1474-1480 
Schiekirka S, Anders S, Raupach T (2014): Assessment of two different types of bias affecting the results of outcome-based evaluation in undergraduate medical education. BMC Med Educ 14, 149

Schiekirka S, Feufel M, Herrmann-Lingen C, Raupach T (2015): Evaluation in medical education: A topical review of target parameters, data collection tools and confounding factors. Ger Med Sci $\underline{13}$, Doc15

Schiekirka S, Raupach T (2015): A systematic review of factors influencing student ratings in undergraduate medical education course evaluations. BMC Med Educ $\underline{15}, 30$

Schiekirka S, Reinhardt D, Beissbarth T, Anders S, Pukrop T, Raupach T (2013): Estimating learning outcomes from pre- and posttest student self-assessments: a longitudinal study. Acad Med $\underline{88}, 369-375$

Schiekirka S, Reinhardt D, Heim S, Fabry G, Pukrop T, Anders S, Raupach T (2012): Student perceptions of evaluation in undergraduate medical education: A qualitative study from one medical school. BMC Med Educ $\underline{12}, 45$

Schmelkin LP, Spencer KJ, Gellman ES (1997): Faculty Perspectives on Course and Teacher Evaluations. Res High Educ $\underline{38}, 575-592$

Schormair C, Swietlik U, Hofmann U, Wilm S, Witte L (1992): Ten statements on the motivation of medical teachers to teach. Med Teach 14, 283-286

Seabrook MA (2003): Medical teachers' concerns about the clinical teaching context. Med Educ $\underline{37}, 213-222$

Skeff KM (1988): Enhancing teaching effectiveness and vitality in the ambulatory setting. J Gen Intern Med $\underline{3}$, 26-33

van den Heuvel M, Demerouti E, Bakker AB (2014): How psychological resources facilitate adaptation to organizational change. Eur J Work Organ Psychol 23, 847-858

Wibbecke G, Kahmann J, Pignotti T, Altenberger L, Kadmon M (2015): Improving teaching on the basis of student evaluation: integrative teaching consultation. GMS J Med Educ $\underline{32}$, Doc2

Woloschuk W, Coderre S, Wright B, McLaughlin K (2011): What Factors Affect Students' Overall Ratings of a Course? Acad Med $\underline{86}, 640-643$ 


\section{Anhang}

Anhang A: Fragebogen

Anhang B: Exemplarischer Evaluationsfragebogen für die Veranstaltungsform Seminar

Anhang C: Exemplarischer Evaluationsfragebogen für die Veranstaltungsform Unterricht am Krankenbett

Anhang D: Exemplarischer Evaluationsfragebogen für die Veranstaltungsform Vorlesung

Anhang E: Beispielhaftes Exemplar für einen individuellen Feedbackreport

Anhang F: Beispielhaftes individuelles Anschreiben 
Anhang A

MUSTER

\begin{tabular}{l|c|c|c|}
\hline \multicolumn{1}{|c|}{ EvaSys } & Lehre und Lehrbedingungen für klinisch tätige Ärzte/innen & Electric Paper \\
\hline \hline & \\
& \\
\hline Bitte so markieren: & $\square \bigotimes \square \square \square$ Bitte verwenden Sie einen Kugelschreiber oder nicht zu starken Filzstift. Dieser Fragebogen wird maschinell erfasst. \\
Korrektur: & $\square \square \square \square$ Bitte beachten Sie im Interesse einer optimalen Datenerfassung die links gegebenen Hinweise beim Ausfüllen.
\end{tabular}

\section{Einleitung}

Sehr geehrte Lehrende,

vielen Dank, dass Sie sich 10 Minuten Zeit nenmen, aıe roıgenden Fragen zu beantworten. Sie helfen uns damit, Ihre Situation als Lehrende innerhalb der klinischen universitären Medizin besser zu verstehen.

\section{Abschnitt: Zur Person}

Sind Sie als Arzt/Ärztin in der humanmedizinischen Lehre der UMG

tätig?

Geschlecht

Alter

Facharzt

Habilitiert
$\square$ ja
$\square$ nein

$\square$ weiblich $\quad \square$ männlich
$\square$ jünger als 26 Jahre $\square$ 46-55 Jahre

$\square$ 26-35 Jahre

$\square$ 36-45 Jahre

älter als 55 Jahre

$\begin{array}{ll}\square \text { ja } & \square \text { nein } \\ \square \text { ja } & \square \text { nein }\end{array}$
Seit wie vielen Jahren sind Sie als Dozent/in in der Lehre (Studium der Humanmedizin) tätig?

$$
\begin{aligned}
& \square \text { weniger als } \\
& 2 \text { Jahre }
\end{aligned}
$$

$\square$ 6-7 Jahre

$$
\begin{aligned}
& \square \text { 2-3 Jahre } \\
& \square \text { 8-9 Jahre }
\end{aligned}
$$

$\square$ mehr als 9 Jahre

In welchem der folgenden Bereiche sind Sie tätig?
$\square$ Allgemeinmedizin
$\square$ Anästhesiologie

$\square$ Augenheilkunde

$\square$ Frauenheilkunde, Geburtshilfe

$\square$ Chirurgie

$\square$ Hals-NasenOhrenheilkunde

$\square$ Hygiene, Mikrobiologie, $\square$ Innere Medizin Virologie

$\square$ Klinische Chemie, Laboratoriumsdiagnostik

$\square$ Pathologie

Neurologie

Pharmakologie, Toxikologie

$\square$ Psychosomatische Medizin und Psychotherapie

$\square$ sonstiges

Rechtsmedizin

$\square$ Arbeitsmedizin, Sozialmedizin

$\square$ Dermatologie, Venerologie

$\square$ Humangenetik

$\square$ Kinderheilkunde

$\square$ Orthopädie F12565UOP1PLOVO

MUSTER 


\section{MUSTER}

EvaSys

\section{Abschnitt}

Im Folgenden geht es um lhre Idealvorstellung von "guter Lehre".

Bitte bewerten Sie auf einer Skala („sehr unwichtig“ bis „sehr wichtig“), inwiefern die folgenden Aspekte für Sie "gute Lehre“ als Ideal-Zustand charakterisieren.

(1) Der Unterricht macht den Studierenden

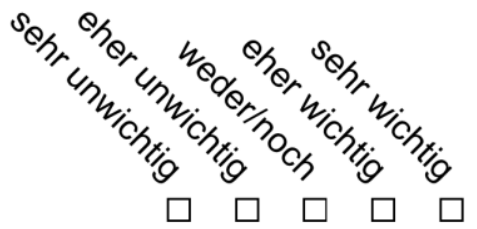
Spaß.

(2) Der Unterricht macht dem Lehrenden Spaß.

(3) Im Unterricht herrscht ein gutes Lernklima.

(4) Inhaltlich ist ein roter Faden erkennbar.

(5) Die Vermittlung von Theorie und Praxis ist ausgewogen.

(6) Im Unterricht werden nicht nur Wissensinhalte sondern auch praktische Fertigkeiten und professionelle Haltungen vermittelt.

(7) Die Lehrenden stimmen sich untereinander ab.

(8) Die Veranstaltungsform (Seminar, Vorlesung, Unterricht am Krankenbett etc.) ist auf die Lernziele und Lerninhalte abgestimmt.

(9) Der/die Lehrende berücksichtigt das Vorwissen der Studierenden.

(10) Der/die Lehrende berücksichtigt die Individualität der Studierenden.

(11) Der/die Lehrende schafft bei den Studierenden Begeisterung und Motivation für das Thema.

(12) Der/die Lehrende ist didaktisch geschult.

(13) Bei den Studierenden wird ein hoher Lernerfolg erzielt.

(14) Bei den Studierenden wird ein nachhaltiger Lernerfolg erzielt.

Bitte benennen Sie nun die drei der oben genannten Apekte, die für Sie bezüglich "guter Lehre" höchste Priorität haben.

Wählen Sie dazu im Folgenden die enstpechende Ziffer (1-14), die vor dem jeweiligen Aspekt in Klammern steht, aus. 
2. Abschnitt [Fortsetzung]

Höchste Priorität hat für mich der Aspekt mit der Ziffer:
$\square 1$
$\square 2$
$\square 3$
$\square 4$
$\square 5$
$\square 6$
$\square 7$
$\square 8$
$\square 9$
$\square 10$
$\square 11$
$\square 12$
$\square 13$
$\square 14$

Zweithöchste Priorität hat für mich der Aspekt mit der Ziffer:
$\square 1$
$\square 2$
$\square 3$
$\square 4$
$\square 5$
$\square 6$
$\square 7$
$\square 8$
$\square 9$
$\square 10$
$\square 11$
$\square 12$
$\square 13$
$\square 14$

Dritthöchste Priorität hat für mich der Aspekt mit der Ziffer:

$\begin{array}{lll}\square 1 & \square 2 & \\ \square 4 & \square 5 & \square 3 \\ \square 7 & \square 8 & \square 6 \\ \square 10 & \square 11 & \square 9 \\ \square 13 & \square 14 & \square 12\end{array}$

\section{Abschnitt}

Im Folgenden geht es nun um lhre tatsächliche Lehrsituation.

Bitte schätzen Sie auf einer Skala (,trifft gar nicht zu“ bis "trifft völlig zu“) ein, wie sehr die folgenden Faktoren Sie persönlich bei der Durchführung Îhrer Lehre behindern. -

(1) Fehlende Zeit zur Vorbereitung der Lehre

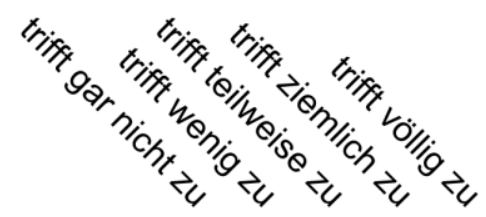
während der Arbeitszeit.

(2) Zu wenig Abstimmung der Lehrenden untereinander.

(3) Zu wenig Möglichkeiten, eigene Konzepte in die Lehre zu integrieren.

(4) Zu geringe eigene didaktische Qualifikation

Bitte benennen Sie nun die drei der oben genannten Faktoren, die Sie am stärksten bei der Durchführung Ihrer Lehre behindern.

Wählen Sie dazu im Folgenden die enstpechende Ziffer (1-4), die vor dem jeweiligen Faktor oben in Klammern steht, aus. 


$\begin{array}{llll}\begin{array}{l}\text { 3. Abschnitt } \\ \text { [Fortsetzung] }\end{array} & & \\ \text { Am stärksten behindert mich der Faktor } & \square 1 & \square 2 & \square 3 \\ \text { mit der Ziffer: } & \square 4 & & \\ \text { Am zweitstärksten behindert mich der } & \square 1 & \square 2 & \square 3 \\ \text { Faktor mit der Ziffer: } & \square 4 & \square 2 & \square 3 \\ \text { Am drittstärksten behindert mich der } & \square 1 & \square & \\ \text { Faktor mit der Ziffer: } & \square 4 & & \end{array}$

Weitere Faktoren, die Sie bei der Durchführung Ihrer Lehre behindern:

Im Folgenden geht es weiterhin um lhre tatsächliche Lehrsituation.

Bitte schätzen Sie nun auf einer Skala („trifft gar nicht zu“ bis „trifft völlig zu“) ein, wie sehr die folgenden Faktoren Sie persönlich bei der Durchfühung Ihrer Lehre unterstützen würden.

(1) Mehr Anerkennung/Würdigung von guter

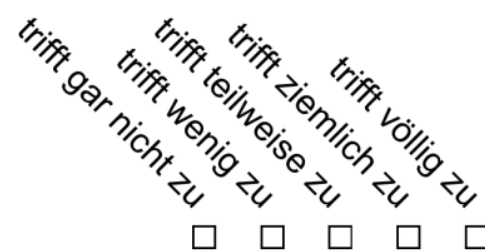
Lehrleistung (z.B. durch die Fakultät,

Abteilungsleiter oder direkte Vorgesetzte)

(2) Ein personenbezogenes Anreizsystem

(3) Positive Rückmeldungen der Studierenden

(4) Freie Zeit als Belohnung für gute Lehre

(5) Zeit zur Vor- und Nachbereitung der Lehre während der Arbeitszeit

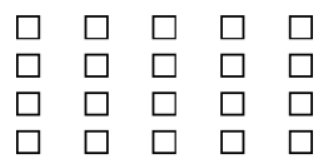

Bitte benennen Sie nun die drei der oben genannten Faktoren, die Sie am stärksten bei der Durchführung Ihrer Lehre unterstützen würden.

Wählen Sie dazu im Folgenden die enstpechende Ziffer (1-5), die vor dem jeweiligen Faktor oben in Klammern steht, aus.

Am stärksten unterstützen würde mich der Faktor mit der Ziffer:

$\square 1$

$\square 4$

$\square 2$

$\square 3$

Am zweitstärksten unterstützen würde

$\square 1$

mich der Faktor mit der Ziffer:

$\square 4$

$\square 5$

$\square 2$

$\square 5$

Am drittstärksten unterstützen würde

$\square 1$

$\square 2$

mich der Faktor mit der Ziffer:

$\square 4$

$\square 5$

$\square 3$ 


\section{Abschnitt}

[Fortsetzung]

Weitere Faktoren, die Sie bei der Durchführung Ihrer Lehre unerstützen würden:

\section{Abschnitt}

Haben Sie bereits an

medizindidaktischen Trainings bzw.

Weiterbildungen teilgenommen?

Gibt es, soweit Sie wissen, medizindidaktische Angebote an Ihrer

Fakultät?

Würden Sie sich gern medizindidaktisch

weiterbilden (möglicherweise auch

zusätzlich zu bereits wahrgenommenen

Weiterbildungen)?

Wenn ja, welche der folgenden Faktoren haben Sie bisher daran gehindert (Mehrfachauswahl möglich)?

$\square$ Es gibt keine Angebote an der eigenen Fakultät.

$\square$ Ich habe keine Zeit daran teilzunehmen. $\square$ Es gibt zwar Angebote, aber die bestehenden Angebote halte ich für wenig hilfreich.

$\square$ Die Angebote sind zu teuer. $\square$ Mein Vorgesetzter unterstützt meine Teilnahme nicht.

$\square$ sonstiges

Sollte die Teilnahme an einer medizindidaktischen Weiterbildung

$\square$ ja $\quad \square$ nein

Voraussetzung für die Habilitation sein?

Bitte bewerten Sie nun auf einer Skala ("gar nicht nützlich" bis "sehr nützlich"), inwiefern die folgenden Bestandteile medizindidaktischer Fortbildungsprogramme für Sie nützlich/hilfreich wären.

Allgemeine Betrachtungen zum
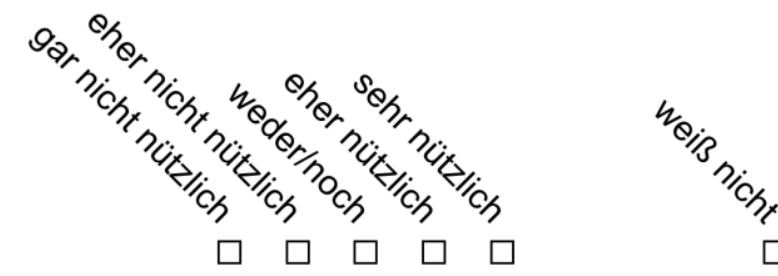

Medizinstudium in Deutschland

Besonderheiten der eigenen

Medizinischen Fakultät

Veranstaltungsplanung 


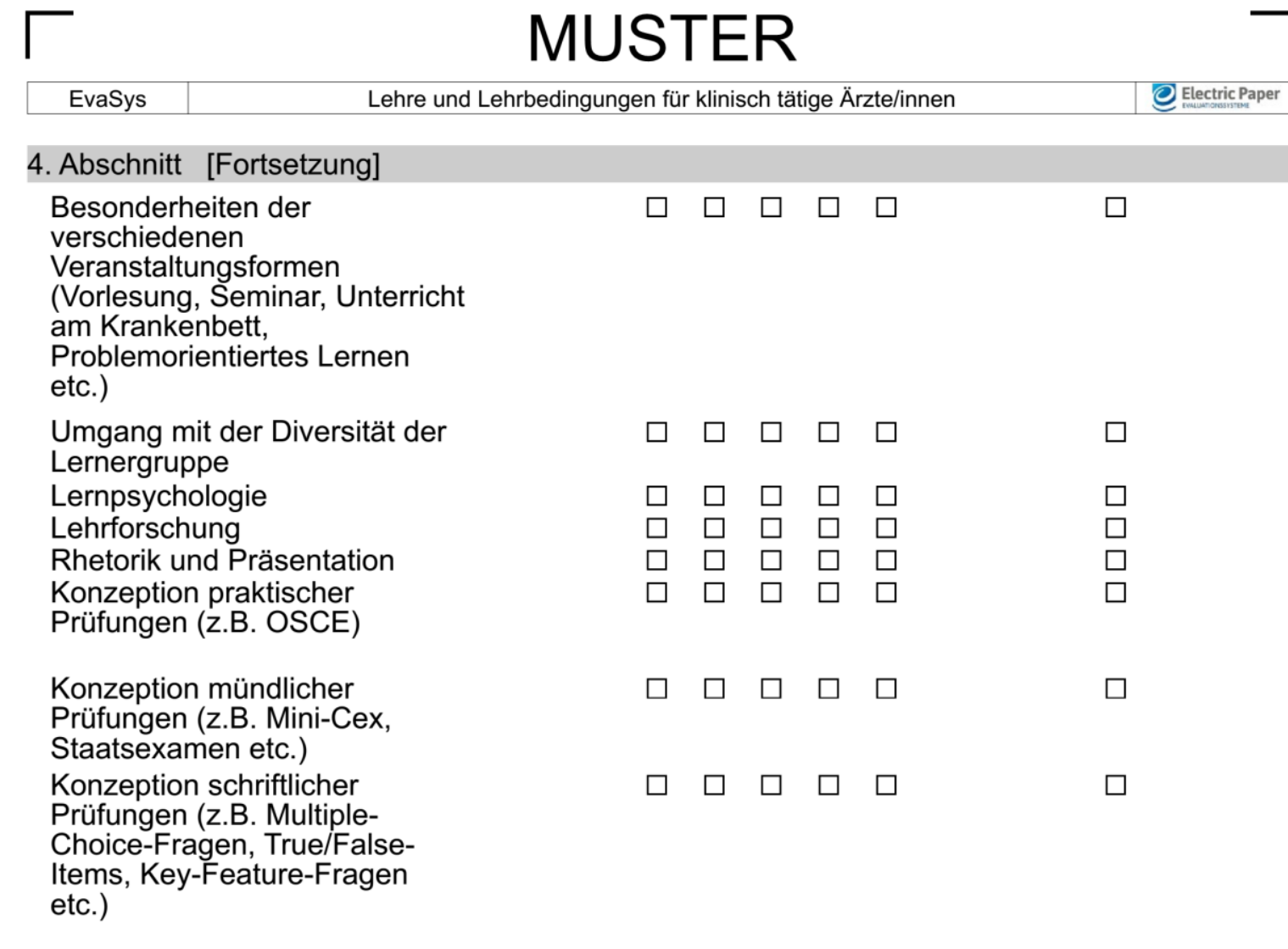

\section{Abschnitt}
Erhalten Sie regelmäßig die
Evaluationsergebnisse Ihrer
$\square$ ja, immer
$\square$ ja,
$\square$ nein, nie

Lehrveranstaltung/en?

Falls Sie auf die vorherige Frage mit "ja, immer" oder "ja, manchmal" geantwortet haben:

Welchen Nutzen haben für Sie die Evaluationsdaten (Mehrfachauswahl möglich)?
$\square$ Feedbackfunktion
$\square$ Verbesserung meiner
$\square$ Qualitätssicherung
$\square$ Vergleich mit anderen Lehrenden eigenen Lehre
$\square$ Steigerung meiner eigenen $\quad \square$ gar keinen Nutzen Motivation

Haben Sie schon mal auf der Basis

Ihrer eigenen Evaluationsergebnisse

$\square \mathrm{ja}$

$\square$ nein

konkrete Verbesserungen in der Lehre

umgesetzt? 


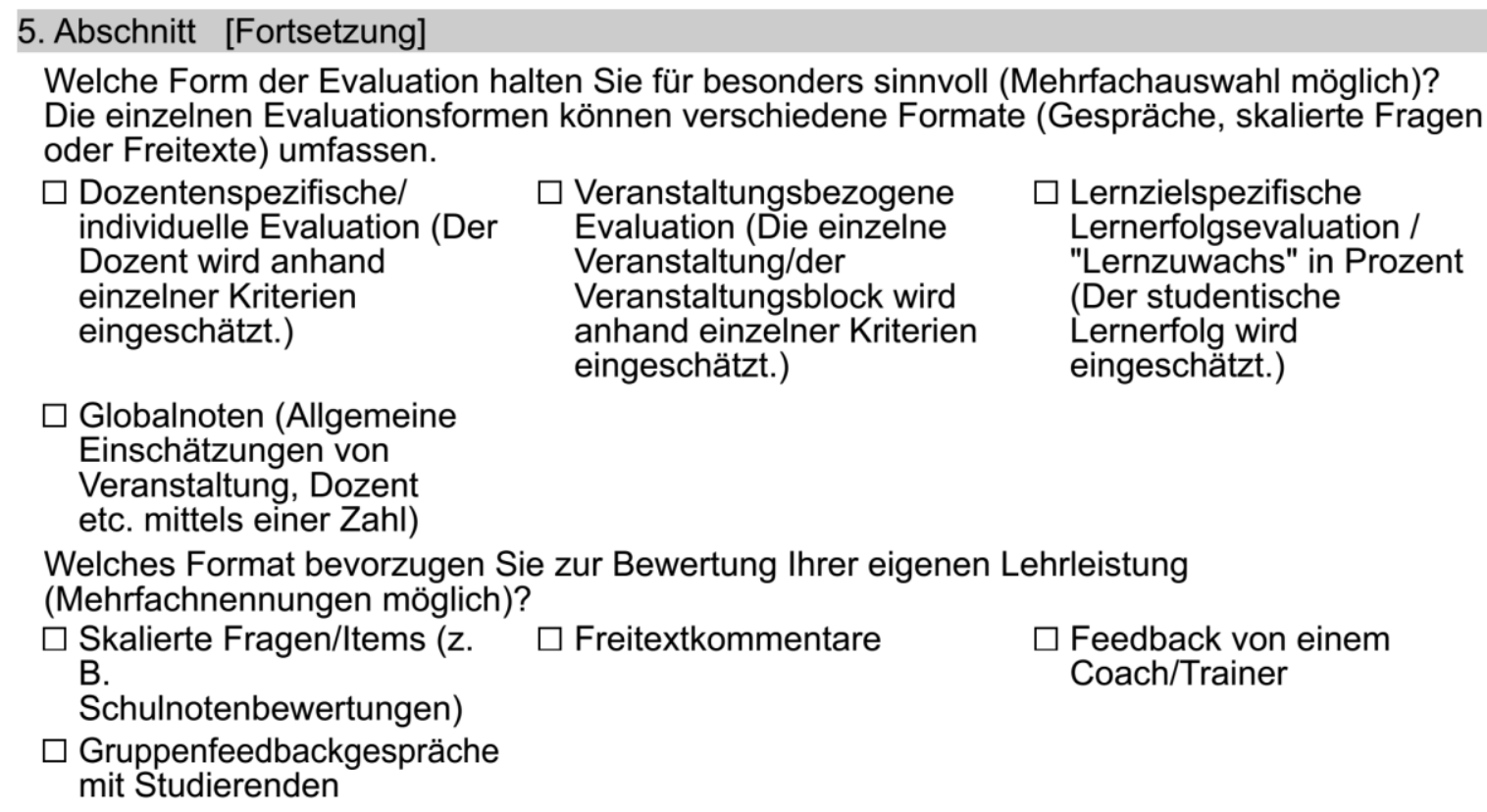

Vielen Dank, dass Sie sich die Zeit genommen haben, diesen Fragebogen auszufüllen. 
Anhang B

\begin{tabular}{l}
\hline \\
\hline EvaSys \\
\hline \hline Universitätsmedizin Göttingen \\
\hline $\begin{array}{l}\text { Bitte so markieren: } \\
\text { Korrektur: }\end{array}$ \\
\hline
\end{tabular}

Liebe Studierende,

bitte beurteilen Sie die von Ihnen besuchte Lehrveranstaltung anhand der folgenden Aussagen. Denken Sie dabei an alle Tutorial-Termine bei diesem Dozenten bzw. dieser Dozentin innerhalb des Moduls, an dem Sie gerade teilnehmen.

Bitte lesen Sie jede Aussage aufmerksam durch und beurteilen Sie, wie sehr Sie dieser Aussage zustimmen.

Mit der sorgfältigen Beantwortung dieses Fragebogens unterstützen Sie gute Lehre.

Bitte machen Sie pro Aussage nur ein Kreuz in das Kästchen, dessen Antwort am ehesten auf Sie zutrifft.

\section{Personenbezogene Daten}

1.1 Bitte geben Sie Ihr Geschlecht an: $\quad \square$ männlich $\square$ weiblich

\section{Fragen zur Lehrveranstaltung}

2.1 In der Veranstaltung herrscht ein gutes Lernklima.

2.2 Der Schwierigkeitsgrad der Veranstaltung ist an den Wissensstand der Studierenden angepasst.

2.3 Der in der Veranstaltung bearbeitete Stoffumfang ist angemessen.

2.4 Die Veranstaltung ist klar gegliedert.

2.5 Die vom Dozenten/von der Dozentin während der Veranstaltung eingesetzten Materialien und Medien (z.B. Folien, Präsentation, Tafel, Film, Präparate, Arbeitsblätter etc.) sind hilfreich für mein Tafel, Film, Präp
Verständnis.

2.6 Die Veranstaltung beinhaltet eine gute Mischung aus Wissensvermittlung durch den Dozenten/die Dozentin und aktiver Beteiligung der Studierenden.

\section{Der/Die Dozent/in}

2.7 - verhält sich den Studierenden gegenüber respektvoll.

2.8 - geht auf Fragen und Beiträge der Studierenden ausreichend ein.

2.9 - gibt den Studierenden konstruktives Feedback.

2.10 - stellt die zentralen Inhalte (konkrete Lernziele) zu Beginn der Veranstaltung dar.

2.11 - stellt Verständnisfragen, um die gelernten Inhalte abzusichern.

2.12 - gestaltet die Veranstaltung so, dass mein Interesse am Themengebiet gefördert wird.

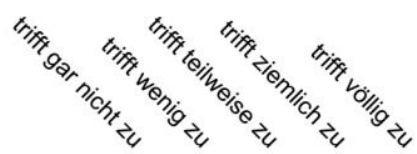

$\square \square \square \square \square$

$\square \square \square \quad \square \quad \square$

$\square \square \square \square$

$\square \square \square \square \square \square$

$\square \square \square \square$

$\square \quad \square \quad \square \quad \square$ 
2. Fragen zur Lehrveranstaltung [Fortsetzung]

Der/Die Dozent/in

2.13 - veranschaulicht die Inhalte anhand von anwendungsbezogenen/praxisnahen Beispielen.

2.14 - drückt sich verständlich aus.

2.15 - moderiert und ergänzt Beiträge von Studierenden (z.B. Referate,

Gruppenarbeiten) in sinnvoller Weise.

2.16 Wie bewerten Sie den Dozenten/die Dozentin insgesamt?

(1)

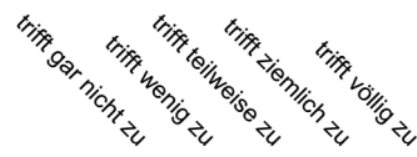

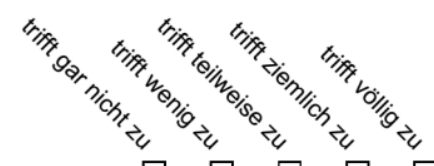

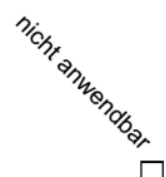

$\square$

$$
\text { sehrgut } \begin{array}{llllll}
\square & \sim & \omega & \overrightarrow{ } & \text { r } & \\
& \square & \square & \square & \begin{array}{l}
\text { überhaupt } \\
\text { nicht gut }
\end{array}
\end{array}
$$

\section{Ihre Kommentare}

3.1 In folgendes Freitextfeld können Sie weitere Anregungen und Anmerkungen eintragen (z.B.: Lob/Kritik an der Lehrveranstaltung Anmerkungen zum Fragebogen selbst, Verbesserungsvorschläge etc.). 


\section{MUSTER}

EvaSys

\section{Ausgewählte Lernziele}

Dieser Evaluationsbogen wird zur Qualitätskontrolle des Lernerfolgs in allen Modulen eingesetzt.

Zu jeder der folgenden Aussagen möchten wir Sie um ZWEI Einschätzungen bitten:

Zuerst kreuzen Sie bitte Ihren aktuellen Leistungsstand an und danach Ihren Leistungsstand zu Modulbeginn - das heißt, Sie sollen aus heutiger Sicht rückblickend beurteilen, wie Ihr eigener Leistungsstand direkt VOR Modulbeginn gewesen ist.

Ich kann alle therapeutischen Akutmaßnahmen benennen, die bei einer COPD-Exazerbation ergriffen werden müssen.

4.1 - aktuelle Selbsteinschätzung: trifft voll zu $\square \square \square$ trifft über-

- rückblickende Selbsteinschätzung zu Modulbeginn:

trifft voll zu

haupt nicht zu

4.

Ich kann die Pathophysiologie der Lungenembolie im Detail beschreiben.

4.3 - aktuelle Selbsteinschätzung:

trifft voll zu

$\square \square \square \square \square \square$ trifft über-

- rückblickende Selbsteinschätzung zu Modulbeginn

trifft voll zu

haupt nicht zu

4.4

Ich kann den Wells-Score genau berechnen und richtig interpretieren.

4.5 - aktuelle Selbsteinschätzung

trifft voll zu

$\square \square \square \square \square \square \quad$ trifft über-

rifft über-

haupt nicht zu

4.6 - rückblickende Selbsteinschätzung zu Modulbeginn:

trifft voll zu

haupt nicht zu

haupt nicht z

Ich kann den diagnostischen Algorithmus, der bei Patienten mit Verdacht auf Lungenembolie angewendet werden muss, korrekt wiedergeben.

4.7 - aktuelle Selbsteinschätzung:

trifft voll zu

$\square \square \square \square \square \square$ trifft über-

4.8 - rückblickende Selbsteinschätzung zu Modulbeginn: trifft voll zu

Ich kann die Therapie der Wahl einer akuten Lungenembolie in Abhängigkeit vom Sterblichkeitsrisiko in der Akutsituation detailliert erläutern.

4.9 - aktuelle Selbsteinschätzung:

trifft voll zu

trifft über-

4.10 - rückblickende Selbsteinschätzung zu Modulbeginn:

trifft voll zu

haupt nicht Zu

Ich kann das Ergebnis einer D-Dimer-Bestimmung im klinischen Kontext korrekt interpretieren.

4.11 - aktuelle Selbsteinschätzung:

4.12 - rückblickende Selbsteinschätzung zu Modulbeginn:

Ich kann auf dem Boden der Labor- und EKG-Diagnostik die Entitäten

4.13 - aktuelle Selbsteinschätzung:

4.14 - rückblickende Selbsteinschätzung zu Modulbeginn: trifft voll zu

trifft voll zu trifft voll zu

trifft über-

haupt nicht zu

trifft über-

haupt nicht zu

trifft über-

haupt nicht zu

rifft über-

Ich kann mit Hilfe wiederholter Troponin-Messungen das Management von Patienten mit akutem Koronarsyndrom fachgerecht planen.

4.15 - aktuelle Selbsteinschätzung:

trifft voll zu

trifft über-

4.16 - rückblickende Selbsteinschätzung zu Modulbeginn:

trifft voll zu

haupt nicht Zu

Ich kann die zeitliche Kinetik der Herzenzyme nach Beginn eines akuten Myokardinfarkts detailliert beschreiben.

4.17 - aktuelle Selbsteinschätzung:

trifft voll zu

haupt nicht zu

4.18 - rückblickende Selbsteinschätzung zu Modulbeginn:

trifft voll zu

trifft über-

haupt nicht zu

trifft über-

haupt nicht zu 


\section{MUSTER}

\begin{tabular}{l|l} 
EvaSys & Lehrevaluation - Seminar
\end{tabular}

4. Ausgewählte Lernziele [Fortsetzung]

Ich kann die Definition der NYHA-Stadien der chronischen Linksherzinsuffizienz detailliert wiedergeben.

4.19 - aktuelle Selbsteinschätzung: trifft voll zu

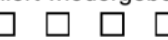

4.20 - rückblickende Selbsteinschätzung zu Modulbeginn:

trifft voll zu

trifft über-

haupt nicht zu

Untersuchung fo

trifft über-

haupt nicht zu

Ich kann eine klinische Fragestellung für eine echokardiographische Untersuchung formulieren (Eintrag in das entsprechende Feld auf der Untersuchungs-Anforderung).

4.21 - aktuelle Selbsteinschätzung:

trifft voll zu

trifft über-

4.22 - rückblickende Selbsteinschätzung zu Modulbeginn:

trifft voll zu

haupt nicht zu

Ich kann die stadienabhängige medikamentöse Therapie der chronischen systolischen Herzinsuffizienz im Detail wiedergeben.

4.23 - aktuelle Selbsteinschätzung

trifft voll zu

trifft über-

4.24 - rückblickende Selbsteinschätzung zu Modulbeginn:

trifft voll zu

haupt nicht zu

trifft über-

haupt nicht zu

Ich kann die Definitionen des paroxysmalen, persistierenden und permanenten Vorhofflimmerns im Detail wiedergeben.

4.25 - aktuelle Selbsteinschätzung:

trifft voll zu

4.26 - rückblickende Selbsteinschätzung zu Modulbeginn:

trifft voll zu

haupt nicht zu

Ich kann den CHA2DS2-VASc-Score korrekt anwenden.

4.27 - aktuelle Selbsteinschätzung:

trifft voll zu

trifft über-

haupt nicht zu

4.28 - rückblickende Selbsteinschätzung zu Modulbeginn:

trifft voll zu

trifft über-

haupt nicht zu

Ich kann den Begriff "ductus-abhängiger Herzfehler" detailliert erläutern.

4.29 - aktuelle Selbsteinschätzung:

trifft voll zu

trifft über-

haupt nicht zu

4.30 - rückblickende Selbsteinschätzung zu Modulbeginn:

trifft voll zu

trifft über-

haupt nicht zu

Ich kann die Hämodynamik beim Vorhofseptumdefekt genau erläutern

4.31 - aktuelle Selbsteinschätzung:

trifft voll zu

trifft über-

haupt nicht zu

4.32 - rückblickende Selbsteinschätzung zu Modulbeginn:

trifft voll zu

trifft über-

haupt nicht Zu

trifft über-

haupt nicht zu

VIELEN DANK FÜR IHRE UNTERSTÜTZUNG!!! 
Anhang C

MUOSTER

\begin{tabular}{l|l} 
EvaSys & Lehrevaluation - UaK
\end{tabular}

Bitte so markieren: $\square \mathbb{X} \square \square$ Bitte verwenden Sie einen Kugelschreiber oder nicht zu starken Filzstift. Dieser Fragebogen wird maschinell erfasst.

Korrektur: $\quad \square \square \square \bigotimes \square$ Bitte beachten Sie im Interesse einer optimalen Datenerfassung die links gegebenen Hinweise beim Ausfüllen.

Liebe Studierende,

bitte beurteilen Sie die von Ihnen besuchte Lehrveranstaltung anhand der folgenden Aussagen. Denken Sie dabei an alle UaK-Termine bei diesem Dozenten bzw. dieser Dozentin innerhalb des Moduls, an dem Sie gerade teilnehmen.

Bitte lesen Sie jede Aussage aufmerksam durch und beurteilen Sie, wie sehr Sie dieser Aussage zustimmen. Mit der sorgfältigen Beantwortung dieses Fragebogens unterstützen Sie gute Lehre.

Bitte machen Sie pro Aussage nur ein Kreuz in das Kästchen, dessen Antwort am ehesten auf Sie zutrifft.

1. Personenbezogene Daten
1.1 Bitte geben Sie Ihr Geschlecht an: $\quad \square$ männlich $\quad \square$ weiblich

\section{Fragen zur Lehrveranstaltung}

2.1 Der Schwierigkeitsgrad der Veranstaltung ist an den

Wissensstand der Studierenden angepasst.

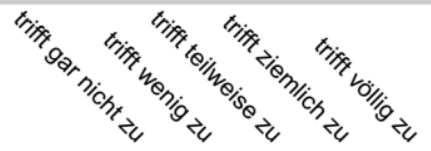

2.2 Der in der Veranstaltung bearbeitete Stoffumfang ist angemessen.

2.3 Die Veranstaltung ist klar gegliedert.

2.4 Die in der Veranstaltung behandelten Inhalte stimmen mit den zu Beginn dargestellten Lernzielen überein.

2.5 Die Veranstaltung beinhaltet eine gute Mischung aus

Wissensvermittlung durch den Dozenten/die Dozentin und aktive Beteiligung der Studierenden.

2.6 In der Veranstaltung habe ich ausreichend Gelegenheit, Patienten zu untersuchen.

Der/Die Dozent/in

2.7 - verhält sich den Studierenden gegenüber respektvoll.

2.8 - geht auf Fragen und Beiträge der Studierenden ausreichend ein.

2.9 - gibt den Studierenden konstruktives Feedback.

2.10 - stellt die zentralen Inhalte (konkrete Lernziele) zu Beginn der Veranstaltung dar.

2.11 - gibt mir Rückmeldung über meine praktischen Fertigkeiten bei der Untersuchung von Patienten.

2.12 - gestaltet die Veranstaltung so, dass mein Interesse am Themengebiet gefördert wird. 


\section{Fragen zur Lehrveranstaltung [Fortsetzung]}

Der/Die Dozent/in

2.13 - gibt mir ausreichend Gelegenheit, mein theoretisches Wissen praktisch anzuwenden.

2.14 - erläutert die Befunde der körperlichen Untersuchung.

2.15 - trainiert gemeinsam mit den Studierenden klinische Untersuchungsmethoden.

2.16 - veranschaulicht klinische Zusammenhänge an Patienten.

2.17 - drückt sich verständlich aus.

2.18 - erscheint pünktlich.

2.19 - geht angemessen mit Störungen des Ablaufs um.

(

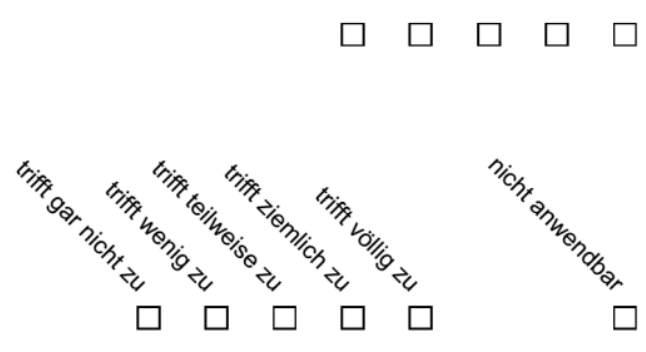

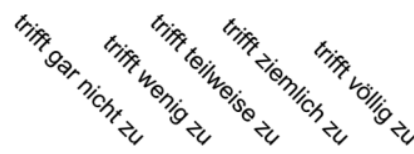

$\square \quad \square \quad \square \quad \square$

$\square \quad \square \quad \square \quad \square \quad \square$

$\square \square \square \square \square$

$\square \square \square \square$

$\square \square \square \quad \square \quad \square$

$\square \square \square \quad \square \quad \square$
2.20 Wie bewerten Sie den Dozenten/ die Dozentin insgesamt?
sehrgut $\square \quad \square \quad \square \quad \square \quad \square \quad$ überhaupt

\section{Ihre Kommentare}

3.1 In folgendes Freitextfeld können Sie weitere Anmerkungen und Anregungen eintragen (z.B. Lob/ Kritik an der Lehrveranstaltung, Anmerkungen zum Fragebogen, Verbesserungsvorschläge etc.). 


\section{MUSTER}

EvaSys

\section{Ausgewählte Lernziele}

Dieser Evaluationsbogen wird zur Qualitätskontrolle des Lernerfolgs in allen Modulen eingesetzt.

Zu jeder der folgenden Aussagen möchten wir Sie um ZWEI Einschätzungen bitten:

Zuerst kreuzen Sie bitte Ihren aktuellen Leistungsstand an und danach Ihren

Leistungsstand zu Modulbeginn - das heißt, Sie sollen aus heutiger Sicht rückblickend beurteilen, wie Ihr eigener Leistungsstand direkt VOR Modulbeginn gewesen ist.

Bitte beachten Sie die veränderten Antwortmöglichkeiten von "trifft voll zu" bis "trifft überhaupt nicht zu".

Ich kann alle wesentlichen Aspekte benennen, die bei der Anamnese von Patienten mit koronarer Herzkrankheit zu berücksichtigen sind.

4.1 - aktuelle Selbsteinschätzung:

trifft voll zu $\square \square \square \square \square \square$ trifft über-

- rückblickende Selbsteinschätzung zu Modulbeginn:

trifft voll zu haupt nicht zu

4.2 kann eine vollständige Raucheranamnese erheben.

4.3 - aktuelle Selbsteinschätzung:

trifft voll zu $\square \square \square \square \square \square \quad$ trifft über-

haupt nicht zu

4.4 - rückblickende Selbsteinschätzung zu Modulbeginn:

trifft voll zu

trifft über-

haupt nicht zu

Ich kann alle wesentlichen Aspekte benennen, die bei der Anamnese von Patienten mit Herzinsuffizienz zu berücksichtigen sind 4.5 - aktuelle Selbsteinschätzung: trifft voll zu trifft über-

4.6 - rückblickende Selbsteinschätzung zu Modulbeginn:

trifft voll zu haupt nicht zu haupt nicht zu

Ich kann alle wesentlichen Aspekte benennen, die bei der Anamnese von Patienten mit Verdacht auf Herzrhythmusstörungen zu berücksichtigen sind.
4.7 - aktuelle Selbsteinschätzung:
4.8 - rückblickende Selbsteinschätzung zu Modulbeginn:

trifft voll zu

trifft voll zu

trifft über-

haupt nicht zu

trift voll zu

trifft über-

haupt nicht zu

Ich kann die klinische Manifestation der chronischen Mitralklappeninsuffizienz detailliert beschreiben.
4.9 - aktuelle Selbsteinschätzung:
trifft voll zu
trifft über-
4.10 - rückblickende Selbsteinschätzung zu Modulbeginn:
trifft voll zu
trifft über-
haupt nicht zu

Ich kann alle typischen Stellen zur Palpation der peripheren Pulse demonstrieren.

4.11 - aktuelle Selbsteinschätzung: trifft voll zu

4.12 - rückblickende Selbsteinschätzung zu Modulbeginn:

trifft voll zu

trifft über-

haupt nicht zu

trift voll zu

trifft über-

haupt nicht zu

Ich kann den Jugularvenendruck mit Hilfe eines Lagerungsverfahrens genau bestimmen.
4.13 - aktuelle Selbsteinschätzung
trifft voll zu
trifft über-
haupt nicht zu
4.14 - rückblickende Selbsteinschätzung zu Modulbeginn:
trifft voll zu
trifft über-

haupt nicht zu 


\section{$\ulcorner$ MUSTER}

EvaSys

\section{Ausgewählte Lernziele [Fortsetzung]}

Ich kann eine Thoraxperkussion fachgerecht durchführen.

4.15 - aktuelle Selbsteinschätzung:
4.16 - rückblickende Selbsteinschätzung zu Modulbeginn: 4.17 - aktuelle Selbsteinschätzung:

4.18 - rückblickende Selbsteinschätzung zu Modulbeginn:

\section{trifft voll zu}

trifft voll zu trifft über-

haupt nicht zu

trifft über-

haupt nicht zu

4.19 - aktuelle Selbsteinschätzung:

trifft voll zu

trifft voll zu

trifft voll zu

$\square \square \square \square \square \square \quad$ trifft über-

haupt nicht zu

trifft über-

haupt nicht zu

trifft voll zu

trifft über-

haupt nicht zu

4.20 - rückblickende Selbsteinschätzung zu Modulbeginn:

trifft über-

haupt nicht zu

Ich kann im Detail darlegen, welche Diagnostik bei einem Patienten mit Verdacht auf KHK indiziert ist.

4.21 - aktuelle Selbsteinschätzung:

trifft voll zu

$\square \square \square \square$

trifft voll zu

$\square \quad$ trifft über-

haupt nicht zu

4.22 - rückblickende Selbsteinschätzung zu Modulbeginn:

trift voll zu

trifft über-

haupt nicht zu

Ich kann eine klinische Fragestellung für eine echokardiographische Untersuchung formulieren (Eintrag in das entsprechende Feld auf der Untersuchungs-Anforderung).

4.23 - aktuelle Selbsteinschätzung:

trifft voll zu

trifft über-

4.24 - rückblickende Selbsteinschätzung zu Modulbeginn:

trifft voll zu

VIELEN DANK FÜR IHRE UNTERSTÜTZUNG!!! 
Anhang D

\begin{tabular}{l}
\hline \begin{tabular}{|l|l|l|}
\hline EvaSys & Lehrevaluation - Vorlesung \\
\hline \hline Universitätsmedizin Göttingen & Electric Paper \\
\hline Bitte so markieren: \\
Korrektur:
\end{tabular}$\square \square \square \square$ Bitte verwenden Sie einen Kugelschreiber oder nicht zu starken Filzstift. Dieser Fragebogen wird maschinell erfasst. \\
\hline
\end{tabular}

Liebe Studierende,

bitte beurteilen Sie die von Ihnen besuchte Lehrveranstaltung anhand der folgenden Aussagen. Denken Sie dabei an alle Vorlesungstermine bei diesem Dozenten bzw. dieser Dozentin innerhalb des Moduls, an dem Sie gerade teilnehmen.

Bitte lesen Sie jede Aussage aufmerksam durch und beurteilen Sie, wie sehr Sie dieser Aussage zustimmen. Mit der sorgfältigen Beantwortung dieses Fragebogens unterstützen Sie gute Lehre.

Bitte machen Sie pro Aussage nur ein Kreuz in das Kästchen, dessen Antwort am ehesten auf Sie zutrifft.

\section{Personenbezogene Daten}

1.1 Bitte geben Sie Ihr Geschlecht an:

$\square$ männlich

$\square$ weiblich

\section{Fragen zur Lehrveranstaltung}

2.1 Der Schwierigkeitsgrad der Veranstaltung ist an den Wissensstand der Studierenden angepasst.

2.2 Der in der Veranstaltung bearbeitete Stoffumfang ist angemessen.

2.3 Die Veranstaltung ist klar gegliedert.

2.4 Die vom Dozenten/von der Dozentin während der Veranstaltung eingesetzten Materialien und Medien (z.B. Folien, Präsentation, Tafel, Film, Präparate, Arbeitsblätter etc.) sind hilfreich für mein Verständnis.

2.5 Die in der Veranstaltung behandelten Inhalte stimmen mit den zu Beginn dargestellten Lernzielen überein.

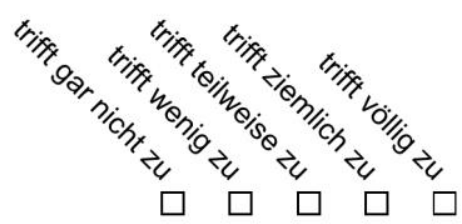

6 Die Veranstaltung beinhaltet eine gute Mischung aus Wissensvermittlung durch den Dozenten/die Dozentin und aktiver Beteiligung der Studierenden.

\section{Der/Die Dozent/in}

2.7 - verhält sich den Studierenden gegenüber respektvoll.

2.8 - geht auf Fragen und Beiträge der Studierenden ausreichend ein.

2.9 - stellt die zentralen Inhalte (konkrete Lernziele) zu Beginn der Veranstaltung dar. 
2. Fragen zur Lehrveranstaltung [Fortsetzung]

\section{Der/Die Dozent/in}

2.10 - stellt Verständnisfragen, um die gelernten Inhalte abzusichern.

2.11 - gestaltet die Veranstaltung so, dass mein Interesse am Themengebiet gefördert wird.

2.12 - macht Zusammenhänge deutlich.

2.13 - veranschaulicht die Inhalte anhand von anwendungsbezogenen/ praxisnahen Beispielen.

2.14 - drückt sich verständlich aus.

2.15 Wie bewerten Sie den Dozenten/ die Dozentin insgesamt?
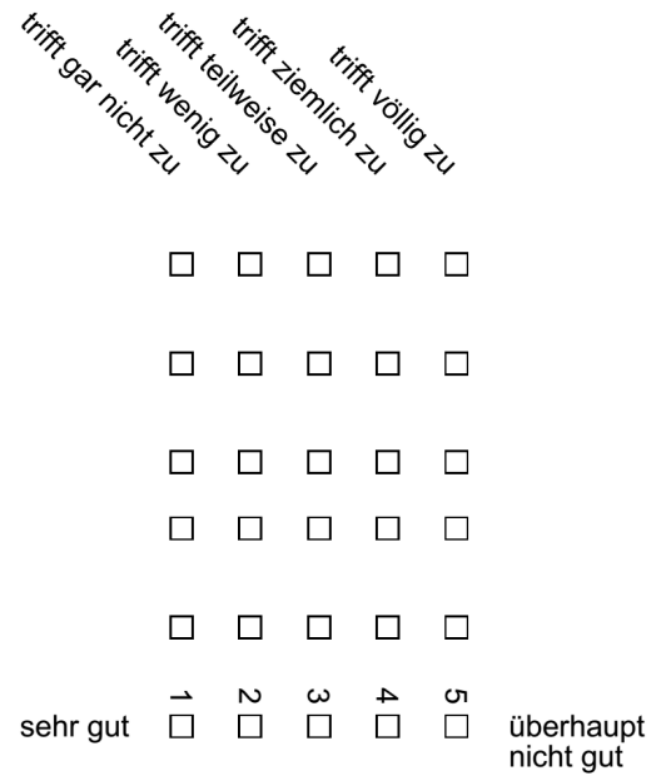

\section{Ihre Kommentare}

3.1 In folgendes Freitextfeld können Sie weitere Anmerkungen und Anregungen eintragen (z.B. Lob/ Kritik an der Lehrveranstaltung, Anmerkungen zum Fragebogen, Verbesserungsvorschläge etc.). 


\section{Ausgewählte Lernziele}

Dieser Evaluationsbogen wird zur Qualitätskontrolle des Lernerfolgs in allen Modulen eingesetzt.

Zu jeder der folgenden Aussagen möchten wir Sie um ZWEI Einschätzungen bitten:

Zuerst kreuzen Sie bitte Ihren aktuellen Leistungsstand an und danach Ihren Leistungsstand zu

Modulbeginn - das heißt, Sie sollen aus heutiger Sicht rückblickend beurteilen, wie Ihr eigener

Leistungsstand direkt VOR Modulbeginn gewesen ist.

Ich kann die Begriffe „Koronarinsuffizienz" und „Koronarreserve“ detailliert erläutern.

4.1 - aktuelle Selbsteinschätzung: trifft voll zu

\section{$\square \quad \square \quad$ trifft über-} haupt nicht zu

4.2 - rückblickende Selbsteinschätzung zu Modulbeginn:

trifft voll zu

trifft über-

haupt nicht zu

Ich kann die pathogenetischen Faktoren, die zu einer Koronarinsuffizienz führen können, im Detail erläutern.

4.3 - aktuelle Selbsteinschätzung:

trifft voll zu

trifft über-

haupt nicht $\mathrm{zu}$

4.4 - rückblickende Selbsteinschätzung zu Modulbeginn:

trifft voll zu

trifft überhaupt nicht $\mathrm{zu}$

Ich kann im Detail darlegen, welche Diagnostik bei einem Patienten mit Verdacht auf KHK indiziert ist. 4.5 - aktuelle Selbsteinschätzung: trifft voll zu trifft überhaupt nicht zu

4.6 - rückblickende Selbsteinschätzung zu Modulbeginn:

trifft voll zu

trifft überhaupt nicht zu 


$\lceil\quad$ MUSTER

4. Ausgewählte Lernziele

[Fortsetzung]

VIELEN DANK FÜR IHRE UNTERSTÜTZUNG! 
Evaluation Ihres UaKs in Modul XX in SSXX

\begin{tabular}{|c|c|c|}
\hline & $\begin{array}{l}\text { Alle Dozenten } \\
\text { (Bewertungen } \\
\text { von } 79 \\
\text { Studierenden) }\end{array}$ & $\begin{array}{l}\text { Dozent } X \\
\text { (Bewertungen } \\
\text { von } 21 \\
\text { Studierenden) }\end{array}$ \\
\hline & Mittelwert \pm SD & Mittelwert \pm SD \\
\hline \multicolumn{3}{|c|}{ Aussagen zur Veranstaltung } \\
\hline $\begin{array}{l}\text { Der Schwierigkeitsgrad der Veranstaltung ist } \\
\text { angemessen. }\end{array}$ & $4,36 \pm 0,69$ & $4,70 \pm 4,47$ \\
\hline $\begin{array}{l}\text { Der in der Veranstaltung bearbeitete Stoffumfang ist } \\
\text { angemessen. }\end{array}$ & $4,23 \pm 0,72$ & $4,50 \pm 0,61$ \\
\hline Die Veranstaltung ist klar gegliedert. & $3,97 \pm 0,93$ & $4,05 \pm 1,03$ \\
\hline $\begin{array}{l}\text { Die in der Veranstaltung behandelten Inhalte stimmen } \\
\text { mit den zu Beginn dargestellten Lernzielen überein. }\end{array}$ & $4,06 \pm 0,78$ & $4,40 \pm 0,75$ \\
\hline $\begin{array}{l}\text { Die Veranstaltung beinhaltet eine gute Mischung aus } \\
\text { Wissensvermittlung durch den Dozenten/die Dozentin } \\
\text { und aktiver Beteiligung der Studierenden. }\end{array}$ & $4,53 \pm 0,71$ & $4,81 \pm 0,40$ \\
\hline $\begin{array}{l}\text { In der Veranstaltung habe ich ausreichend Gelegenheit, } \\
\text { Patienten zu untersuchen. }\end{array}$ & $4,11 \pm 0,92$ & $3,48 \pm 1,08$ \\
\hline \multicolumn{3}{|c|}{ Aussagen zur didaktischen Kompetenz: „Der/Die Dozent/in...“ } \\
\hline ...verhält sich den Studierenden gegenüber respektvoll. & $4,85 \pm 0,46$ & $5,00 \pm 0,00$ \\
\hline $\begin{array}{l}\text {...geht auf Fragen und Beiträge der Studierenden } \\
\text { ausreichend ein. }\end{array}$ & $4,82 \pm 0,47$ & $5,00 \pm 0,00$ \\
\hline ...gibt den Studierenden konstruktives Feedback. & $4,43 \pm 0,76$ & $4,67 \pm 0,58$ \\
\hline ...stellt die Lernziele zu Beginn der Veranstaltung dar. & $3,97 \pm 1,03$ & $4,24 \pm 0,89$ \\
\hline $\begin{array}{l}\text {...überprüft meine praktischen Fertigkeiten bei der } \\
\text { Untersuchung von Patienten. }\end{array}$ & $3,83 \pm 1,03$ & $4,15 \pm 0,81$ \\
\hline $\begin{array}{l}\text {...gestaltet die Veranstaltung so, dass mein Interesse } \\
\text { am Themengebiet gefördert wird. }\end{array}$ & $4,48 \pm 0,81$ & $4,78 \pm 0,44$ \\
\hline $\begin{array}{l}\text {...gibt mir ausreichend Gelegenheit, mein theoretisches } \\
\text { Wissen praktisch anzuwenden. }\end{array}$ & $4,08 \pm 0,72$ & $4,14 \pm 0,85$ \\
\hline ...erläutert die Befunde der körperlichen Untersuchung. & $4,49 \pm 0,68$ & $4,60 \pm 0,50$ \\
\hline ... erklärt klinische Untersuchungsmethoden. & $4,09 \pm 0,86$ & $3,95 \pm 0,97$ \\
\hline $\begin{array}{l}\text {... veranschaulicht klinische Zusammenhänge an } \\
\text { Patienten. }\end{array}$ & $4,47 \pm 0,66$ & $4,76 \pm 0,44$ \\
\hline ...drückt sich verständlich aus. & $4,73 \pm 0,60$ & $5,00 \pm 0,00$ \\
\hline ...erscheint pünktlich. & $4,32 \pm 0,97$ & $4,52 \pm 0,81$ \\
\hline ... geht angemessen mit Störungen des Ablaufs um. & $4,73 \pm 0,53$ & $4,92 \pm 0,28$ \\
\hline Gesamtnote & $1,49 \pm 0,64$ & $1,24 \pm 0,44$ \\
\hline \multicolumn{3}{|c|}{$\begin{array}{l}\text { Legende: } \\
\text { Aussagen: } 5=\text { trifft völlig zu; } 4=\text { trifft ziemlich } z u ; 3=\text { trifft teilweise } z u ; 2=\text { trifft wenig zu; } 1=\text { triff } \\
\text { gar nicht zu } \\
\text { Gesamtnote: von } 1 \text { (sehr gut) bis } 5 \text { (mangelhaft) }\end{array}$} \\
\hline
\end{tabular}




\section{Studentischer Lernerfolg}

\begin{tabular}{|c|c|c|}
\hline & $\begin{array}{l}\text { Alle Dozenten } \\
\text { (Daten von } 79 \\
\text { Studierenden) }\end{array}$ & $\begin{array}{l}\text { Dozent X } \\
\text { (Daten von } 21 \\
\text { Studierenden) }\end{array}$ \\
\hline \multicolumn{3}{|l|}{ Lernziele } \\
\hline $\begin{array}{l}\text { Ich kann alle wesentlichen Aspekte benennen, die } \\
\text { bei der Anamnese von Patienten mit Herzinsuffizienz } \\
\text { zu berücksichtigen sind. }\end{array}$ & $70,80 \%$ & $79,59 \%$ \\
\hline $\begin{array}{l}\text { Ich kann auf dem Boden einer Anamnese die NYHA- } \\
\text { Klasse eines Patienten mit Herzinsuffizienz } \\
\text { festlegen. }\end{array}$ & $79,66 \%$ & $75,86 \%$ \\
\hline $\begin{array}{l}\text { Ich kann alle typischen klinischen Symptome und } \\
\text { Befunde der chronischen Linksherzinsuffizienz } \\
\text { aufzählen. }\end{array}$ & $67,12 \%$ & $65,22 \%$ \\
\hline $\begin{array}{l}\text { Ich kann alle typischen klinischen Symptome und } \\
\text { Befunde der chronischen Rechtsherzinsuffizienz } \\
\text { aufzählen. }\end{array}$ & $68,82 \%$ & $69,23 \%$ \\
\hline $\begin{array}{l}\text { Ich kann alle Parameter benennen, auf die bei der } \\
\text { Inspektion des Thorax geachtet werden muss. }\end{array}$ & $62,37 \%$ & $60,87 \%$ \\
\hline Ich kann den Herzspitzenstoß sicher palpieren. & $53,16 \%$ & $44,83 \%$ \\
\hline $\begin{array}{l}\text { Ich kann die Auskultationsareale der einzelnen } \\
\text { Herzklappen exakt demonstrieren. }\end{array}$ & $70,07 \%$ & $72,22 \%$ \\
\hline $\begin{array}{l}\text { Ich kann alle typischen Stellen zur Palpation der } \\
\text { peripheren Pulse demonstrieren. }\end{array}$ & $62,07 \%$ & $61,29 \%$ \\
\hline $\begin{array}{l}\text { Ich kann eine klinische Fragestellung formulieren, die } \\
\text { mein weiteres Vorgehen in der körperlichen } \\
\text { Untersuchung leitet. }\end{array}$ & $58,33 \%$ & $59,68 \%$ \\
\hline $\begin{array}{l}\text { Ich kann im Detail darlegen, bei welchen Patienten } \\
\text { die Durchführung einer kapillären oder arteriellen } \\
\text { BGA indiziert ist. }\end{array}$ & $33,49 \%$ & $33,75 \%$ \\
\hline $\begin{array}{l}\text { Ich kann eine klinische Fragestellung für eine } \\
\text { echokardiographische Untersuchung formulieren } \\
\text { (Eintrag in das entsprechende Feld auf der } \\
\text { Untersuchungs-Anforderung). }\end{array}$ & $56,04 \%$ & $60,00 \%$ \\
\hline $\begin{array}{l}\text { Ich kann eine klinische Fragestellung für eine } \\
\text { Röntgenuntersuchung des Thorax formulieren } \\
\text { (Eintrag in das entsprechende Feld auf der } \\
\text { Untersuchungs-Anforderung). }\end{array}$ & $51,86 \%$ & $45,31 \%$ \\
\hline
\end{tabular}

Der Lernerfolg kann zwischen $-100 \%$ und $+100 \%$ betragen. Bei der Berechnung wird das studentische Vorwissen berücksichtigt.

grüne Kästen: Lernerfolg um mehr als $10 \%$ besser als bei der gesamten Dozentengruppe

\author{
gelbe Kästen: Lernerfolg \\ nicht wesentlich höher I \\ niedriger als bei der \\ gesamten Dozentengruppe
}

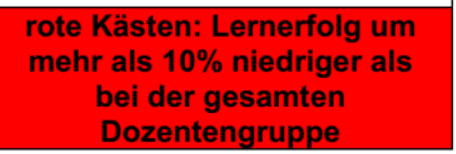




\section{Freitextkommentare}

- Sehr guter Dozent/Verständlicher Erklärungen \& nette Betreuung/ Weiter so!

- Sehr interaktiv/ Insgesamt im Studium zu wenig Patientenkontakt

- Intensiv-Station war sehr interessant. / Die Untersuchung (Prae-OP) vom 2ten Tag war gut strukturiert und gewinnbringend.

- Tag auf der Intensivstation sehr interessant. Danke für den Einblick.

- Dozent: Sehr motiviert, fragt viel, beantwortet gerne Fragen/ Veranstaltung: wenig strukturiert, kein regelmäßiger Besuch von Patienten, keine aktive Untersuchung von Kindern

- Sehr Studierendenfreundlicher Umgang! / Ist schon gut sehr gut auf Wünsche der Studierenden eingegangen.

- Der Dozent hat den Stoff anschaulich und sehr gut erklärt. Weiter so.

- Sehr gute Veranstaltung, sehr gut umgesetzt. / Herr X ist ein sehr kompetenter und engagierter Dozent!

- Sehr angenehme Atmosphäre, Fragen sehr ausführlich beantwortet, Feedback erfragt, was Studenten am UaK interessiert und vielseitige Patientenfälle herausgesucht / evtl. kleinere Gruppen zum Patienten, damit jeder mehr Fragen kann, aber insgesamt wirklich super UaK.

- Noch mehr Patienten untersuchen!

- Danke für den sehr interessanten Uak!

- Dozent ist sehr engagiert + organisiert. Insgesamt [Handschrift nicht lesbar] 


\title{
Ihre Ergebnisse der Lehrveranstaltungsevaluation im SoSe 2015
}

\author{
Sehr geehrte/r Frau/Herr X, \\ im Sommersemester 2015 haben Sie uns dabei unterstützt, den von uns neu entwickelten \\ Fragebogen zur Qualität der Lehre weiter zu testen. Dafür möchten wir uns bei Ihnen erst \\ einmal herzlich bedanken.
}

Im Anhang dieser E-Mail finden Sie Ihre persönlichen Ergebnisse. Sie waren im Sommersemester als UaK-Dozent/in eingesetzt. In der angehängten Datei finden Sie zunächst (linke Spalte) die Mittelwerte der Angaben aller Studierenden, die an UaKs teilgenommen und ihre Lehrenden evaluiert haben - es handelt sich also um die Mittelwerte der Bewertungen aller Lehrenden der gleichen Unterrichtsform. In der rechten Spalte finden Sie Ihre persönlichen Ergebnisse. Dabei haben wir diejenigen Werte markiert, die besonders gut ausgefallen sind (grün) bzw. bei denen noch Verbesserungspotential besteht (rot). Damit wollen wir Sie dabei unterstützen, spezifische Stärken und Schwächen Ihrer Lehre zu identifizieren.

Alle UaK-Dozenten/innen wurden nicht nur hinsichtlich ihrer didaktischen Kompetenz evaluiert, sondern die Studierenden wurden auch um Angaben zu ihrem inhaltichen Lernerfolg gebeten. Der studentische Lernerfolg wird nach der in Göttingen entwickelten Formel als Prozentwert angegeben (für Details zur Berechnung siehe Schiekirka et al. Acad Med 2013; 88).

Des Weiteren finden Sie im Anhang die Freitextkommentare der Studierenden zu Ihren Veranstaltungen. Wir haben diese wörtlich übernommen und möchten darauf hinweisen, dass diese Kommentare nur Einzelmeinungen widerspiegeln können.

Insgesamt ergibt sich aus Ihren individuellen Evaluationsergebnissen folgendes Bild:

- Besondere Stärken:

- Berücksichtigung von Fragen und Beiträgen der Studierenden

- Gute Mischung aus aktiver Beteiligung und Wissensvermittlung

- Förderung des Interesses

- Bereiche mit Entwicklungspotential:

- Die Gelegenheit zur Untersuchung von Patienten

○ Erklärung klinischer Untersuchungsmethoden

Wenn Sie Interesse daran haben, möchten wir Sie gerne dabei unterstützen, Ihre Lehrkompetenz weiter auszubauen. Falls Sie die Evaluationsergebnisse mit uns diskutieren wollen oder sich Anregungen wünschen, wie der eine oder andere Aspekt gestärkt werden kann, kontaktieren Sie uns bitte unter medizindidaktik@med.uni-goettingen.de.

Natürlich interessiert uns auch, ob diese ausführliche Evaluation für Sie einen besonderen Nutzen hat. Diesbezüglich werde ich Sie in den nächsten Wochen telefonisch kontaktieren und nach Ihrer Meinung fragen. Unser Ziel ist es, mit möglichst geringem Aufwand die für die Optimierung Ihrer Lehre benötigten Informationen zu gewinnen und Ihnen zukommen zu lassen.

Wir möchten noch einmal darauf hinweisen, dass ausschließlich Sie persönlich diese Auswertung erhalten. Im Projektteam wurden die Ergebnisse lediglich in anonymisierter 
Form diskutiert, und außer den Projektmitarbeitern, die diese Daten für Sie zusammengestellt haben, hat niemand Ihre persönliche Auswertung gesehen.

Zeitnah werden wir Ihnen die Daten auch zusätzlich noch in Papierform zusenden.

Bei Rückfragen können Sie uns jederzeit gerne kontaktieren.

Mit freundlichen Grüßen,

Dipl.-Psych. Sarah Schiekirka

Medizindidaktik Göttingen 


\section{Publikationen}

1. Schiekirka-Schwake S, Anders S, von Steinbüchel N, Becker JC, Raupach T (2017): Facilitators of high-quality teaching in medical school: findings from a nation-wide survey among clinical teachers. BMC Med Educ $\underline{17}, 178$

2. Schiekirka-Schwake S, Dreiling K, Pyka K, Anders S, von Steinbüchel N, Raupach T (2018): Improving evaluation at two medical schools. Clin Teach $\underline{15}$, 314-318

Aufgrund exklusiver Nutzungsrechte des Verlages ist die zweite Publikation nicht in die Dissertation eingebunden. 


\title{
Facilitators of high-quality teaching in medical school: findings from a nation- wide survey among clinical teachers
}

\author{
S. Schiekirka-Schwake ${ }^{1 *} \mathbb{D}$, S. Anders ${ }^{2}$, N. von Steinbüchel ${ }^{3}$, J. C. Becker ${ }^{4}$ and T. Raupach ${ }^{1,5}$
}

\begin{abstract}
Background: Clinical teachers in medical schools are faced with the challenging task of delivering high-quality patient care, producing high-impact research and contributing to undergraduate medical education all at the same time. Little is known on the gap between an 'ideal' environment supporting clinical teachers to provide high quality teaching for their students and the reality of clinical teaching during worktime in the clinical environment. Most quantitative research published so far was done in a wide range of medical educators and did not consider individual academic qualifications. In this study, we wanted to survey clinical teachers in particular and assess the potential impact of individual academic qualification on their perceptions.
\end{abstract}

Methods: Based on qualitative data of focus group discussions, we developed a questionnaire which was piloted among 189 clinical teachers. The final web-based questionnaire was completed by clinical teachers at nine German medical schools.

Results: A total of 833 clinical teachers (569 junior physicians, 264 assistant professors) participated in the online survey. According to participants, the most important indicator of high quality teaching was "sustained student learning outcome" followed by "stimulation of interest in the subject matter". Lack of time was the main factor impeding effective teaching (78\%). Among the factors facilitating high-quality teaching, protected preparation time during working hours (48\%) and more recognition of high-quality teaching within medical schools (21\%) were perceived as most helpful. Three out of four teachers (76\%) were interested in faculty development programmes directed at teaching skills, but $60 \%$ stated they had no time to engage in such activities. With regard to evaluation, teachers preferred individual feedback (75\%) over global ratings (21\%). Differences between assistant professors and junior physicians were found in that the latter group perceived their teaching conditions as more difficult.

Conclusions: Lack of time is a major barrier against planning and delivering good clinical teaching in medical schools. According to our findings, the situation at German medical schools is particularly challenging for junior physicians. Creating an institutional culture in which teaching is regarded as highly as patient care and research is a prerequisite for overcoming the barriers identified in this study.

Keywords: Undergraduate medical education, Clinical teacher, Academic status, Survey, Questionnaire, Barriers, Facilitators, Didactic training, Faculty development, Evaluation

\footnotetext{
* Correspondence: sarah.schiekirka@med.uni-goettingen.de

'Division of Medical Education Research and Curriculum Development, Study

Deanery of Göttingen Medical School, Göttingen, Germany

Full list of author information is available at the end of the article
} 


\section{Background}

Undergraduate medical education needs to meet high standards, and medical schools carry the responsibility to equip future physicians with knowledge, skills and attitudes needed to provide state-of-the-art care for their patients. A considerable amount of teaching in the clinical phase of medical education is delivered by physicians working in university hospitals. Not all of them may have chosen this work environment because they wanted to become clinical teachers, and in most medical schools the completion of a didactic training programme is not a prerequisite for being allowed to teach medical students. But even if they are motivated and adequately prepared to engage in teaching activities, clinical teachers are faced with different challenges as they are expected to produce highimpact research, contribute to medical education and deliver high-quality patient care, virtually all at the same time [1]. Few studies have identified a beneficial impact of teaching assignments on physicians: Hartley et al. [2] reported a positive effect on general practitioners' attitudes following contact with students. In addition, teaching might reduce stress related to the clinical workload [3]. However, a much more common finding is that medical teachers struggle with their teaching role and face a lot of difficulties [4-10]: For instance, semi-structured interviews in 22 teachers in the United Kingdom revealed concerns about insufficient support and institutional recognition of teaching as well as the inability to influence decisions related to medical education [5]. In an Australian study, clinical teachers expressed worries about the quality of patient care as educational activities intervened with their clinical duties [4]. Furthermore, an international web survey including 860 participants revealed a lack of academic recognition and financial support as well as a specific need for didactic trainings as main challenges for medical educators $(n=860)$ [7]. Consistent with this finding, 147 German medical teachers named poor academic recognition (53.5\%) as well as low institutional (31.5\%) and financial (28.4\%) support as important challenges in medical education [6].

Available studies on the subject are heterogeneous regarding their methodology and participants: Some authors used qualitative methods $[4,5,8,9]$, which provide useful information but usually rely on very small samples thus limiting generalisability. Most quantitative research published so far was done in a wide range of medical educators including basic scientists, psychologists, physiotherapists and nurses [7]. Given that the professional background (e.g. medical, nursing, psychology) and the work setting (e.g. university hospital, private sector) is likely to impact perceptions of the teaching environment, restricting survey samples to clinical teachers appears useful for addressing specific research questions. Physicians involved in undergraduate medical teaching are usually assigned to various teaching formats including bedside teaching, small-group discussions, case-based learning, problem-based learning and lectures. Thus, research on their perceptions of the teaching environment should not be confined to one specific format $[8,10]$.

Physicians working in German university medical centres can be divided into two groups: junior physicians (from graduation to board registration) and assistant professors (mostly consultants experienced in research). Junior physicians are primarily involved in patient care. As both teaching experience and research output are required to progress to the stage of an assistant professor, their workload is particularly high. In contrast, assistant professors hold positions with more responsibility and self-determination.

It is generally assumed that all physicians working in university hospitals strive not only to provide high-quality patient care and contribute to advance medical research but also to help their students become excellent physicians themselves. However, data supporting this notion are scarce. In fact, there is no uniform definition of 'highquality teaching, and although a number of facilitators of good teaching have been described, it is unknown whether junior physicians and assistant professors share the same views on what supports and what deters them from delivering high-quality teaching.

The aim of this study was to elicit clinical teachers' understanding of high-quality teaching characteristics and to identify facilitators and barriers of delivering high-quality teaching. In addition, we assessed teachers' perceptions of evaluation and their motivation to participate in didactic trainings. Moreover, we wanted to investigate the impact of academic qualification on clinical teachers' views.

\section{Methods}

\section{Undergraduate medical education in Germany}

There are two different models for undergraduate medical education in Germany: 'Traditional' curricula are made up of a two-year pre-clinical phase and a threeyear clinical phase. In so-called 'reformed' curricula, preclinical and clinical teaching are integrated throughout the first 5 years. In both models, the sixth year comprises three elective periods lasting 4 months each [11]. Clinical teaching is an important feature of both models, thus curriculum type was not considered a major independent variable for this study.

\section{Development and piloting of the questionnaire}

Due to the fact that no suitable questionnaire was available for our research question we developed a new instrument.

Physicians involved in undergraduate medical education at one German medical school (Göttingen) were invited to participate in focus group discussions addressing their definition of high-quality teaching, perceptions of the actual 
teaching environment, evaluation processes and issues related to clinical training. Teachers of all specialties were included and both junior physicians and assistant professors were invited to participate. During spring 2014, four focus group sessions including five to seven participants each were conducted. Two groups were made up of junior physicians ( $n=15$; nine male), and two groups included assistant professors ( $n=11$; all male). Sessions were moderated by one of the authors (SS). Results were categorised based on qualitative content analysis [12] using MaxQDA (VERBI GmbH, Marburg, Germany). Trigger questions served as an orientation for coding, and subthemes were identified in an iterative process. Themes and subthemes were subsequently included in mind maps.

Based on the themes that emerged from focus group discussions we developed a questionnaire containing scaled items. Following iterative discussions with experts in questionnaire development, a first draft of the questionnaire was used for cognitive debriefings with five clinical teachers (three female, two male; three junior physicians, two assistant professors) and adjusted according to their comments.

In January 2015, the web-based questionnaire was piloted in 183 clinical teachers (114 junior physicians (57 male), 69 assistant professors (49 male); overall response rate 26\%) at Göttingen Medical School. Due to ceiling effects for some items addressing the importance of facilitators and barriers (i.e., strong agreement was found for a number of items), we added questions asking participants to rank order the items according to their perceived importance.

The final questionnaire comprised six sections: (1) demographics (seven items), (2) characteristics of highquality teaching (17 items), (3) barriers against delivering high-quality teaching (eight items), (4) factors facilitating high-quality teaching (nine items), (5) didactic training experience (16 items), (6) utility of evaluation results for the improvement of teaching quality (five items). In addition to the 34 scaled items (5-point likert scale with one indicating the most negative and five indicating the most positive response), there were 26 categorical items and two open questions. A translated version of the questionnaire is provided in the Additional file 1.

Between March 2015 and February 2016, study deaneries of medicals schools were contacted by one author (SS) and invited to take part in the survey. Finally, nine medical schools (situated in eight of the 16 German federal states) agreed to participate (between September 2015 and July 2016). The invitation including the introduction to the project and the link to the web-based questionnaire (EvaSys, Electric Paper, Lüneburg, Germany) were sent to eligible clinical teachers by the respective study deanery. All participating study deaneries used comprehensive mailing lists including contact data of a wide range of faculty members. Unfortunately, the exact number of clinical teachers actually working in university hospitals could not be derived from this list, thus thwarting the calculation of a response rate.

\section{Data analysis}

Descriptive analysis of survey data was performed by computing percentages and calculating mean values $(\mathrm{M}) \pm$ standard deviations (SD), as appropriate. Independent $t$-tests, $x^{2}$ tests and effect size measures (Cohen's d) were used to compare data obtained from junior physicians to survey responses provided by assistant professors. Significance levels were set to $5 \%$. Owing to the explorative nature of all analyses, Bonferroni corrections were not used.

\section{Ethics approval}

The Institutional Review Board at Göttingen University Medical Centre (application number 20/4/14) waived ethics approval as the study protocol was not deemed to represent bio-medical or epidemiological research. We made every effort to comply with data protection rules. Study participation was voluntary and all data were collected anonymously.

\section{Results}

Six of the participating medical schools taught in 'traditional' and three in 'reformed' curricula. All of them offered didactic training programmes for their faculty and determined that participation in didactic trainings is a prerequisite for promotion to a position equivalent to a lecturer (e.g. assistant professor).

A total of 1035 subjects completed the questionnaire. Of these, 106 were excluded because they indicated not to be involved in patient care. Another 96 were excluded because they did not provide information on their academic qualification. Thus, a total of 833 questionnaires were analysed (569 junior physicians (313 male); 264 assistant professors (187 male)). Table 1 presents participant characteristics by academic status (junior physicians vs. assistant professors).

All other results are reported below, according to the thematic order of subjects in the questionnaire:

\section{1) Characteristics of high-quality teaching: Ratings} for the 14 scaled items on characteristics of highquality teaching were heavily skewed towards the 'very important' anchor of the rating scale (e.g., high and sustained student learning outcome, good learning climate, students as well as teachers enjoy teaching sessions; see Table 2). While mean ratings for most items were $>4.0$ in both groups, the acknowledgement of individual differences between students was deemed less important by both junior physicians $(3.41 \pm 0.91)$ and assistant professors $(3.44 \pm 0.98)$. Significant differences in ratings between the two 
Table 1 Participant characteristics (grouped by academic status). Discrepancies in numbers result from missing values

\begin{tabular}{|c|c|c|c|}
\hline & & $\begin{array}{l}\text { Junior } \\
\text { physicians } \\
(n=569)\end{array}$ & $\begin{array}{l}\text { Assistant } \\
\text { professors } \\
(n=264)\end{array}$ \\
\hline \multirow[t]{2}{*}{ Sex } & Female & 245 & 71 \\
\hline & Male & 313 & 187 \\
\hline \multirow{2}{*}{$\begin{array}{l}\text { Specialty } \\
\text { Board } \\
\text { Certification }\end{array}$} & Yes & 302 & 247 \\
\hline & No & 264 & 17 \\
\hline \multirow[t]{3}{*}{ Age } & $\leq 35$ years & 272 & 10 \\
\hline & $36-45$ years & 186 & 87 \\
\hline & $>45$ years & 97 & 160 \\
\hline \multirow{2}{*}{$\begin{array}{l}\text { Teaching } \\
\text { Experience }\end{array}$} & $\leq 5$ years & 325 & 10 \\
\hline & $>5$ years & 239 & 253 \\
\hline \multirow[t]{6}{*}{ Specialty } & Anaesthesiology & 79 & 10 \\
\hline & General Medicine \& Paediatrics & 48 & 27 \\
\hline & Internal Medicine & 104 & 36 \\
\hline & Neurology \& Psychiatry & 67 & 29 \\
\hline & $\begin{array}{l}\text { Surgery, Orthopaedics \& } \\
\text { Urology }\end{array}$ & 87 & 46 \\
\hline & $\begin{array}{l}\text { Other (includes Gynaecology, } \\
\text { Ophthalmology, Pharmacology, } \\
\text { Toxicology, Pathology, } \\
\text { Dermatology etc.) }\end{array}$ & 89 & 50 \\
\hline
\end{tabular}

groups were found for "Teachers enjoy teaching/ learning activities" $(\mathrm{t}(830)=3.94, p<0.001)$ and

"Student learning outcome is high" $(\mathrm{t}(829)=2.94$, $p<0.001)$ in that assistant professors assigned greater importance to both aspects compared to junior physicians.

When asked to indicate their individual most important characteristic of high-quality clinical teaching, 38\% of the teachers chose "Student learning outcome is sustainable" followed by "Teacher motivates students and increases their enthusiasm for the subject matter" (16\%) and "Students enjoy teaching/learning activities." (12\%). No significant differences between the two groups were found.

\section{2) Barriers against delivering high-quality teaching:} According to teacher ratings, the most important barrier against delivering high-quality teaching was a lack of protected preparation time while a lack of opportunity to implement individual approaches to teaching was the least important barrier (see Table 3). Junior physicians felt more strongly than assistant professors that they lacked protected preparation time for teaching $(4.19 \pm 0.98$ vs. $3.88 \pm 1.12$; $\mathrm{t}$ $(450.58)=3.88 ; p<0.001)$ as well as didactic training $(2.68 \pm 1.10$ vs. $2.03 \pm 0.90$; $\mathrm{t}(608.09)=9.03$, $p<0.001)$.
Table 2 Characteristics of high-quality teaching. Items were rated on a 5 -point scale ( 1 = very unimportant; 5 = very important)

\begin{tabular}{|c|c|c|c|}
\hline \multirow[t]{2}{*}{ Item } & \multicolumn{2}{|c|}{$\begin{array}{l}\text { Mean } \pm \text { Standard } \\
\text { Deviation }\end{array}$} & \multirow[t]{2}{*}{$\begin{array}{l}\text { Effect size } \\
\text { (Cohen's d) }\end{array}$} \\
\hline & $\begin{array}{l}\text { Junior } \\
\text { physicians }\end{array}$ & $\begin{array}{l}\text { Assistant } \\
\text { professors }\end{array}$ & \\
\hline $\begin{array}{l}\text { Students enjoy teaching/learning } \\
\text { activities }\end{array}$ & $4.48 \pm 0.68$ & $4.53 \pm 0.70$ & \\
\hline $\begin{array}{l}\text { Teachers enjoy teaching/learning } \\
\text { activities }\end{array}$ & $4.20 \pm 0.78^{a}$ & $4.42 \pm 0.69$ & -0.29 \\
\hline The learning climate is good & $4.60 \pm 0.65$ & $4.60 \pm 0.66$ & \\
\hline Sessions have a clear structure & $4.60 \pm 0.69$ & $4.69 \pm 0.66$ & \\
\hline $\begin{array}{l}\text { Content is presented in a } \\
\text { balanced manner }\end{array}$ & $4.24 \pm 0.79$ & $4.33 \pm 0.82$ & \\
\hline $\begin{array}{l}\text { Both knowledge, skills and } \\
\text { attitudes are being taught }\end{array}$ & $4.42 \pm 0.80$ & $4.39 \pm 0.79$ & \\
\hline $\begin{array}{l}\text { Teachers agree in advance on } \\
\text { the content to be taught }\end{array}$ & $4.23 \pm 0.79$ & $4.16 \pm 0.84$ & \\
\hline $\begin{array}{l}\text { Teaching format is aligned to } \\
\text { learning objectives }\end{array}$ & $4.26 \pm 0.78$ & $4.31 \pm 0.77$ & \\
\hline $\begin{array}{l}\text { Teaching is pitched to the } \\
\text { student level }\end{array}$ & $4.10 \pm 0.83$ & $4.21 \pm 0.79$ & \\
\hline $\begin{array}{l}\text { Teachers acknowledge individual } \\
\text { differences between students }\end{array}$ & $3.41 \pm 0.91$ & $3.44 \pm 0.98$ & \\
\hline $\begin{array}{l}\text { Teacher motivates students and } \\
\text { increases their enthusiasm for } \\
\text { the subject matter }\end{array}$ & $4.45 \pm 0.74$ & $4.51 \pm 0.72$ & \\
\hline $\begin{array}{l}\text { Teachers have received didactic } \\
\text { training }\end{array}$ & $3.97 \pm 0.93$ & $4.05 \pm 0.91$ & \\
\hline $\begin{array}{l}\text { Student learning outcome is } \\
\text { high }\end{array}$ & $4.14 \pm 0.79^{a}$ & $4.31 \pm 0.74$ & -0.23 \\
\hline $\begin{array}{l}\text { Student learning outcome is } \\
\text { sustainable }\end{array}$ & $4.62 \pm 0.69$ & $4.63 \pm 0.69$ & \\
\hline
\end{tabular}

${ }^{a} p<0.05$ for comparisons between junior physicians and assistant professors (independent $t$ test). Effect size (Cohen's $d$ ) reported when $t$ test was significant

Analysis of the rank-ordering item revealed that lack of protected preparation time was the main factor impeding high quality teaching for both groups (chosen by $81 \%$ of all participants).

3) Factors facilitating high-quality teaching: Among the factors facilitating high-quality teaching, protected preparation time and positive student feedback were rated as being more important than individual incentives or time off as a reward for good teaching performance (see Table 3). There was a significant difference in ratings between junior physicians and assistant professors in that the former group was even more interested in protected preparation time ( $4.38 \pm 0.95$ vs. $3.98 \pm 1.18$; $\mathrm{t}(414.24)=4.76, p<0.001)$. Compared to assistant professors, junior physicians more strongly agreed that recognition for good teaching would help them deliver high-quality teaching $(3.75 \pm 1.12$ vs. 
Table 3 Barriers and facilitating factors for the delivery of high quality teaching. Teachers were asked to what extent these factors prevented them from/assisted them in delivering highquality teaching. Items were rated on a 5 -point scale $(1=$ not at all; 5 = very much)

\begin{tabular}{|c|c|c|c|}
\hline \multirow[t]{2}{*}{ Item } & \multicolumn{2}{|c|}{$\begin{array}{l}\text { Mean } \pm \text { Standard } \\
\text { Deviation }\end{array}$} & \multirow[t]{2}{*}{$\begin{array}{l}\text { Effect size } \\
\text { (Cohen's d) }\end{array}$} \\
\hline & $\begin{array}{l}\text { Junior } \\
\text { physicians }\end{array}$ & $\begin{array}{l}\text { Assistant } \\
\text { professors }\end{array}$ & \\
\hline \multicolumn{4}{|l|}{ Part I: Barriers } \\
\hline Lack of preparation time & $4.19 \pm 0.98^{\mathrm{a}}$ & $3.88 \pm 1.12$ & 0.32 \\
\hline $\begin{array}{l}\text { Insufficient coordination } \\
\text { between/among teachers }\end{array}$ & $3.55 \pm 1.01$ & $3.49 \pm 1.11$ & \\
\hline $\begin{array}{l}\text { Lack of opportunity to } \\
\text { implement individual teaching } \\
\text { concepts }\end{array}$ & $2.90 \pm 1.05$ & $2.89 \pm 1.25$ & \\
\hline Lack of didactic training & $2.68 \pm 1.10^{\mathrm{a}}$ & $2.03 \pm 0.90$ & 0.65 \\
\hline \multicolumn{4}{|l|}{ Part II: Facilitating factors } \\
\hline $\begin{array}{l}\text { Recognition for good } \\
\text { teaching performance }\end{array}$ & $3.75 \pm 1.12^{\mathrm{a}}$ & $3.45 \pm 1.28$ & 0.22 \\
\hline Individual incentives & $3.25 \pm 1.19$ & $3.12 \pm 1.30$ & \\
\hline Positive student feedback & $4.10 \pm 0.85$ & $4.10 \pm 0.98$ & \\
\hline $\begin{array}{l}\text { Time off as a reward for high } \\
\text { quality teaching }\end{array}$ & $3.27 \pm 1.32^{\mathrm{a}}$ & $2.75 \pm 1.44$ & 0.40 \\
\hline $\begin{array}{l}\text { Protected preparation time } \\
\text { during working hours }\end{array}$ & $4.38 \pm 0.95^{\mathrm{a}}$ & $3.98 \pm 1.18$ & 0.38 \\
\hline
\end{tabular}

${ }^{a} p<0.05$ for comparisons between junior physicians and assistant professors (independent $t$ test). Effect size (Cohen's d) reported when t test was significant

$3.45 \pm 1.28 ; \mathrm{t}(450.33)=2.95, p<0.001)$ and were more interested in time off as a reward for high quality teaching $(3.27 \pm 1.32$ vs. $2.75 \pm 1.44$; $\mathrm{t}(467.56)=4.93, p<0.001)$.

When asked to rank-order the five facilitating factors suggested in the survey, $49 \%$ (assistant professor $=44 \%$, junior physicians $=51 \%$ ) of participants chose protected preparation time.

4) Didactic training experience: Of all 833 survey participants, 483 (58\%) had ever attended didactic training sessions with a significant difference between assistant professors (80\%) and junior physicians $\left(48 \%\right.$; $\left(\chi^{2}(1, N=828)=77.72 ; p<0.001\right)$. Over $82 \%$ were aware of their institution's training programme, and $78 \%$ expressed a general interest in such programmes (junior physicians $84 \%$; assistant professors 60\%; $\left.\chi^{2}(1, N=813)=68.59 ; p<0.001\right)$. The main reason for not participating in didactic trainings was a lack of time, as indicated by $64 \%$ of physicians (junior physicians 67\%; assistant professors 56\%; $\left.\chi^{2}(1, N=806)=9.07 ; p<0.001\right)$; barriers mentioned less frequently were lack of support from supervisors (12\%; junior physicians $14 \%$, assistant professors $6 \% ; \chi^{2}(1, N=806)=12.26$; $p<0.001)$, the notion that such trainings were not helpful (10\% overall, no significant difference between groups), high cost ( $8 \%$ overall; $p=$ n.s.) and lack of awareness of the availability of trainings (7\%; junior physicians $8 \%$, assistant professors $2 \%$; $\chi^{2}(1$, $N=806)=10.59 ; p<0.001)$. A majority of teachers in both groups ( $76 \%$ overall $(N=817) ; p=$ n.s.) supported the view that participation in didactic trainings should be a prerequisite for promotion to a position equivalent to a lecturer (e.g. assistant professor). Content to be covered in trainings was rated on 5-point scales (see Table 4). Presentation skills were rated as most important by assistant professors $(4.05 \pm 1.51)$ whereas junior physicians were most interested in specific characteristics of teaching formats $(4.04 \pm 1.18)$. Again, ratings were skewed towards the 'very important' anchor of the rating scale. The only significant difference between assistant professors and junior doctors was observed for the item "Overview of the medical education system in Germany" (3.00 \pm 1.29 vs. $3.24 \pm 1.24 ; \mathrm{t}$ $(822)=2.49, p=0.01)$.

5) Utility of evaluation results: Only around one third (32\%) of all survey participants (junior physicians $=26 \%$, assistant professors $=44 \%$ ) indicated to receive their evaluation results on a regular basis, whereas $24 \%$ (junior physicians $=30 \%$, assistant professors $=12 \%$; ) never receive their

Table 4 Perceived utility of topics in didactic trainings. Items were rated on a 5 -point scale ( 1 = very unimportant; 5 = very important)

\begin{tabular}{llll}
\hline Item & \multicolumn{2}{l}{$\begin{array}{l}\text { Mean } \pm \text { Standard } \\
\text { Deviation }\end{array}$} & $\begin{array}{l}\text { Effect size } \\
\text { (Cohen's d) }\end{array}$ \\
\cline { 2 - 3 } & $\begin{array}{l}\text { Junior } \\
\text { physicians }\end{array}$ & $\begin{array}{l}\text { Assistant } \\
\text { professors }\end{array}$ & \\
\hline $\begin{array}{l}\text { Overview of medical education } \\
\text { in Germany }\end{array}$ & $3.00 \pm 1.29^{a}$ & $3.24 \pm 1.24$ & -0.19 \\
$\begin{array}{l}\text { Specific characteristics of the } \\
\text { home institution }\end{array}$ & $3.74 \pm 1.22$ & $3.83 \pm 1.16$ & \\
$\begin{array}{l}\text { Teaching session planning } \\
\text { Specific characteristics of }\end{array}$ & $3.78 \pm 1.26$ & $3.73 \pm 1.20$ & \\
teaching formats & $4.04 \pm 1.18$ & $3.92 \pm 1.19$ & \\
Meeting the needs of a diverse & $3.82 \pm 1.39$ & $3.71 \pm 1.30$ & \\
student population & & & \\
Educational psychology & $3.80 \pm 1.47$ & $3.89 \pm 1.42$ & \\
Medical education research & $3.39 \pm 1.46$ & $3.52 \pm 1.48$ & \\
Presentation skills & $3.96 \pm 0.67$ & $4.05 \pm 1.51$ & \\
$\begin{array}{l}\text { Designing practical } \\
\text { examinations }\end{array}$ & $3.88 \pm 1.28$ & $3.76 \pm 1.38$ & \\
Designing oral examinations & $3.97 \pm 1.32$ & $3.97 \pm 1.28$ & \\
Designing written examinations & $3.83 \pm 1.30$ & $3.83 \pm 1.30$ &
\end{tabular}

${ }^{a} p<0.05$ for comparisons between junior physicians and assistant professors (independent $t$ test). Effect size (Cohen's $d$ ) reported when $t$ test was significant 
results. The differences in frequencies were significant $\left(\chi^{2}(2, N=822)=39: 54 ; p<0.001\right)$. Three in five teachers $(60 \%$ (junior physicians $=55 \%$; assistant professors $=72 \% ; \chi^{2}(1, N=764)=21.21$; $p<0.001)$ ) stated they had tried to improve their teaching based on evaluation results.

Most participants (66\%) mainly used evaluation data as a source of feedback and for the improvement of their teaching (57\%). About half of them (47\%) felt that evaluation results increased their motivation to teach, and just one quarter (24\%) used the data for comparisons with fellow teachers. Differences between assistant professors and junior physicians are displayed in Fig. 1. Significant differences were found for "Quality assurance" $\left(\chi^{2}(1\right.$, $N=828)=15.97 ; p<0.001)$ und "Improvement of teaching" $\left(\chi^{2}(1, N=828)=4.98, p=0.03\right)$.

With regard to type of evaluation, $75 \%$ of survey participants preferred individual evaluations over course evaluations (61\%) and learning outcome evaluations (42\%). Only 20\% favoured global ratings.

\section{Discussion}

Clinical teachers participating in this study had a broad understanding of high-quality teaching. Findings from local focus group discussions and pilot testing of the questionnaire at Göttingen medical school were confirmed in the nation-wide survey. Overall, student learning outcome was deemed to be an important indicator of high-quality teaching. The most salient barrier against delivering good teaching was a lack of protected preparation time. Positive student feedback served as a strong motivating factor while individual incentives appeared to play but a minor role. Lack of time also prevented physician teachers from attending didactic trainings although such trainings were considered very important by a majority. Individual evaluation was preferred and received results were used to improve teaching by most survey participants. Differences between assistant professors and junior physicians were found in that the latter group perceived higher barriers in terms of lack of time, lack of didactic trainings, and lack of support provided by supervisors.

Poor teaching conditions due to competing duties for physician teachers appear to be an international [4-8] problem that has been identified quite some time ago $[1,5]$. It is alarming that despite teaching being an integral part of clinical teachers' workload in university hospitals, there is insufficient institutional support in terms of allocated time for teaching itself, preparation of teaching activities or improving teaching by participation in didactic trainings in many institutions. Moreover, the present data indicate that at least at those German medical schools included in this survey, there is room for improvement with regard to academic recognition of high-quality teaching and providing individual evaluation results to teachers. The comparisons between junior physicians and assistant professors suggest that more support is particularly important during the first years following graduation. Presumably, assistant professors have more possibilities to cope with these conditions due to their experiences (made in undergraduate education as well as made as supervisors in continuing medical education) and a higher grade of self-determination.

Solutions for these deficiencies are long overdue. While care for patients must be the top priority for physicians, universities must also ensure that teaching (i.e. training of future physicians who must be equipped with skills to care for patients themselves) can be adequately delivered. The authors feel that teaching should be supported and valued as equally important as patient care and research. This means there can be no trade-off between either of these goals, but all of them should count towards performance measures of individual clinical teachers.

Protected time for teaching activities (including preparation) is needed. However, in some institutions more staff may be required to meet this goal. Given the economic pressure faced by many university hospitals, the creation of additional jobs with a specific focus on teaching seems unlikely but desirable (e.g. creating roles of "clinical

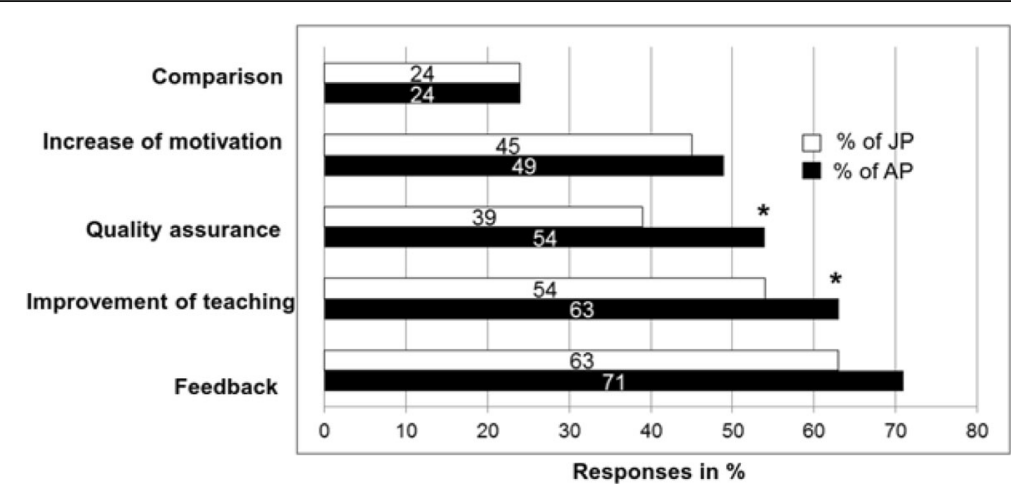

Fig. 1 Utility of evaluation results for physicians (displayed by academic status). Columns represent percentages of survey participants who ticked the respective box on the survey. ${ }^{*} p<0.05$ for the comparison of percentages (junior physicians, JP vs. assistant professors, AP) in a $x^{2}$ test 
educators" whose scope of duties comprise not only teaching but also supporting and supervising junior physicians in their teaching activities). Another possible, more economic strategy could be to involve medical students who are interested in engaging in (peer-)teaching activities.

Furthermore, the establishment of a teaching culture in which academic recognition for excellence in teaching is as high as for outstanding research and patient care may create a stronger motivation for clinical teachers to strive for excellence in teaching. Annual teaching prizes and financial incentives for departments supporting clinical teachers' participation in dedicated training programmes are just a few examples of how perceived value of teaching may be increased.

Additional ways to manage this change in institutional culture need to be identified [13].

\section{Strengths and limitations}

In contrast to previous studies on this topic, we focussed on one specific group of teachers. While this impairs the generalisability to other healthcare professionals involved in medical education, our results help to better understand why physicians sometimes fail to excel in teaching and what needs to be done in order to improve the situation. A particular strength of our study was the sample diversity and size. We included clinical teachers of different medical schools located in eight different German federal states offering different curricula. Moreover, we distinguished between junior physicians and assistant professors.

Interpretation of our results is limited in that only subjective data were assessed and the above-mentioned results and differences between the two groups are based on perceptions only. Furthermore, we could not calculate a definitive response rate, because most participating medical schools were unable to provide exact lists of physicians involved in undergraduate medical education. Due to this fact, we were unable to perform a power analysis; as mentioned above, all statistical comparisons were exploratory in nature. Moreover, because of selfselection of the participating medical schools the sample might not be representative of all clinical teachers at German medical schools.

\section{Conclusion}

Physicians involved in undergraduate medical education face a number of serious barriers against delivering high-quality teaching, especially at the beginning of their career. Creating an institutional culture valuing teaching as no less important than patient care and research is a prerequisite for overcoming the barriers identified in this study.

\section{Additional file}

Additional file 1: Translated version of the Questionnaire. (PDF $133 \mathrm{~kb}$ )

Abbreviations

AP: Assistant professors; JP: Junior physicians; M: Mean value; N: Sample size; SD: Standard deviation; SS: Sarah Schiekirka-Schwake

\author{
Acknowledgements \\ None. \\ Funding \\ There was no funding for this study.
}

Availability of data and materials

The datasets used and/or analysed during the current study available from the corresponding author on reasonable request.

\section{Authors' contributions}

SS designed the study, conducted all interviews, drafted the questionnaire, performed data analysis, contributed to data interpretation and drafted the first version of the manuscript. SA contributed to data interpretation and commented on various versions of the manuscript. NS supervised questionnaire development, contributed to data interpretation and commented on the final version of the manuscript. JB contributed to data presentation and commented on various versions of the manuscript. TR conceived of the study, contributed to data interpretation and commented on various versions of the manuscript. All authors read and approved the final manuscript.

\section{Authors' information}

SARAH SCHIEKIRKA-SCHWAKE is a psychologist at Göttingen University Medical Centre. She is primarily involved in higher education research with a specific focus on evaluation.

SVEN ANDERS works as a consultant in the Department of Legal Medicine at Hamburg University, coordinating the department's teaching activities. Main research areas are forensic pathology, clinical forensic medicine, and medical education.

NICOLE VON STEINBÜCHEL is professor for Medical Psychology and Sociology and director of the Department of Medical Psychology and Medical Sociology in Göttingen. She is a clinical neuropsychologist and human biologist. Her research focuses on psychometric instruments development in various health contexts.

JAN C. BECKER is deputy managing director of the institute of medical education at the medical faculty of Muenster University. His professional and scientific focus is curriculum development.

TOBIAS RAUPACH is professor for medical education research and curriculum development at Göttingen University Medical Centre. He is also a clinical cardiologist. His current research focuses on test-enhanced learning, assessment formats and evaluation.

\section{Ethics approval and consent to participate}

The local Institutional Review Board (application number 20/4/14) waived ethics approval as the study protocol was not deemed to represent bio-medical or epidemiological research. We made every effort to comply with data protection rules. Study participation was voluntary and all data were collected anonymously.

\section{Consent for publication}

No individual's data is included in this study.

\section{Competing interests}

The authors declare that they have no competing interests.

\section{Publisher's Note}

Springer Nature remains neutral with regard to jurisdictional claims in published maps and institutional affiliations. 


\section{Author details}

'Division of Medical Education Research and Curriculum Development, Study Deanery of Göttingen Medical School, Göttingen, Germany. ${ }^{2}$ Department of Legal Medicine, University Medical Centre Hamburg-Eppendorf, Hamburg, Germany. ${ }^{3}$ Institute of Medical Psychology and Medical Sociology, University Medical Centre Göttingen, Göttingen, Germany. ${ }^{4}$ Department of Medical Education, Medical Faculty, University of Münster, Münster, Germany. ${ }^{5}$ Department of Cardiology and Pneumology, University Medical Centre Göttingen, Göttingen, Germany.

Received: 12 May 2017 Accepted: 5 September 2017

Published online: 29 September 2017

\section{References}

1. Abrahamson S. Time to return medical schools to their primary purpose: education. Acad Med. 1996;71(4):343-7.

2. Hartley S, Macfarlane F, Gantley M, Murray E. Influence on general practitioners of teaching undergraduates: qualitative study of London general practitioner teachers. BMJ. 1999;319(7218):1168-71.

3. Rutter $\mathrm{H}$, Herzberg J, Paice E. Stress in doctors and dentists who teach. Med Educ. 2002;36(6):543-9.

4. Barrett J, Scott K. Pedagogical and professional compromises by medical teachers in hospitals. Clin Teach. 2014;11(5):340-4.

5. Seabrook MA. Medical teachers' concerns about the clinical teaching context. Med Educ. 2003;37(3):213-22.

6. Huwendiek S, Hahn EG, Tönshoff B, Nikendei C. Herausforderungen Lehrender in der Medizin: Ergebnisse einer Umfrage unter Mitgliedern der Gesellschaft für Medizinische Ausbildung. GMS Z Med Ausbild. 2013;30(3):Doc38.

7. Huwendiek S, Mennin S, Dern P, Ben-David MF, Van Der Vleuten C, Tonshoff B, Nikendei C. Expertise, needs and challenges of medical educators: results of an international web survey. Med Teach. 2010;32(11):912-8.

8. Arabshahi KS, Haghani F, Bigdeli S, Omid A, Adibi P. Challenges of the ward round teaching based on the experiences of medical clinical teachers. J Res Med Sci. 2015;20(3):273-80.

9. Sabel E, Archer J. "Medical education is the ugly duckling of the medical world" and other challenges to medical educators' identity construction: a qualitative study. Acad Med. 2014:89(11):1474-80.

10. Hoffman KG, Donaldson JF. Contextual tensions of the clinical environment and their influence on teaching and learning. Med Educ. 2004;38(4):448-54.

11. Chenot J-F. Undergraduate medical education in Germany. GMS Ger Med Sci. 2009:7:Doc02.

12. Mayring P. Qualitative Inhaltsanalyse - Grundlagen und Techniken. 11th ed. Beltz Verlag: Weinheim und Basel; 2010.

13. Michie $S$, van Stralen M, West R. The behaviour change wheel: a new method for characterising and designing behaviour change interventions. Implement Sci. 2011;6(1):42.

\section{Submit your next manuscript to BioMed Central and we will help you at every step:}

- We accept pre-submission inquiries

- Our selector tool helps you to find the most relevant journal

- We provide round the clock customer support

- Convenient online submission

- Thorough peer review

- Inclusion in PubMed and all major indexing services

- Maximum visibility for your research

Submit your manuscript at www.biomedcentral.com/submit
Biomed Central 